\title{
LOGARITHMIC COMPACTIFICATION OF THE ABEL-JACOBI SECTION
}

\author{
STEFFEN MARCUS AND JONATHAN WISE
}

\begin{abstract}
Given a smooth curve with weighted marked points, the Abel-Jacboi map produces a line bundle on the curve. This map fails to extend to the full boundary of the moduli space of stable pointed curves. Using logarithmic and tropical geometry, we describe a modular modification of the moduli space of curves over which the Abel-Jacobi map extends. We also describe the attendant deformation theory and virtual fundamental class of this moduli space. This recovers the double ramification cycle, as well as variants associated to differentials.
\end{abstract}

\section{CONTENTS}

1. Introduction

2. The naive Abel-Jacobi map

3. Review of logarithmic geometry

4. The logarithmic compactification of the Abel-Jacobi section

5. Rubber comes from the tropics

References

\section{INTRODUCTION}

Let $C$ be a smooth curve containing distinct marked points $x_{1}, \ldots, x_{n}$. For any vector $\mathbf{a}=\left(a_{1}, \ldots, a_{n}\right)$ of integers, we obtain an invertible sheaf $\mathcal{O}_{C}\left(\sum a_{i} x_{i}\right)$ on $C$. This construction behaves well in families, and gives a section

$$
\mathcal{M}_{g, n} \rightarrow \mathbf{P i c}_{g, n}
$$

where $\mathbf{P i c}_{g, n}$ is the universal Picard group. We call this map the Abel-Jacobi section.

This section fails to extend over $\overline{\mathcal{M}}_{g, n}$. As we will recall in Section 2.1, it does extend to compact type (and even a bit further, though we do not discuss this), but we demonstrate by example in Section 2.2 that it does not extend near a point corresponding to a curve with two components joined at two nodes.

Our main contribution is the observation that, in compact type, the integer weighting a on the marked points of a stable curve $C$ corresponds to an equivalence class of piecewise linear functions on the tropicalization of $C$. This notion makes sense on the boundary of $\overline{\mathcal{M}}_{g, n}$, and in Sections 4.1 and 4.2 we construct a moduli space $\operatorname{Div}_{g, \text { a }}$ parameterizing stable curves with the choice of such a function. We show that this additional datum produces a line bundle on $C$ and that this construction resolves the indeterminacy of the Abel-Jacobi section.

Theorem A. The Abel-Jacobi section extends to a closed embedding aj : Div $\mathbf{g}_{g, \mathbf{a}} \rightarrow \mathbf{P i c}_{g, n}$ over $\overline{\mathcal{M}}_{g, n}$.

The Abel-Jacobi map, as opposed to the Abel-Jacobi section, sometimes refers to the map $\mathcal{M}_{g, \mathbf{a}} \rightarrow \mathbf{P i c}_{g}$, that takes in a smooth curve of genus $g$ with $n$ distinct, weighted markings and outputs the associated line bundle, but forgets the location of the markings. Unlike the Abel-Jacobi section that is the focus of this paper, this Abel-Jacobi map is usually not an embedding. Our methods, along with some technical bookkeeping, also resolve the indeterminacy of the Abel-Jacobi map, as we indicate in Section 4.7. It would be very interesting to understand the fibers of this map, but we make no attempt to do so here.

Theorem A is proven in Section 4.2 where it appears as Theorem 4.3.2. In Section 4.6, we show that $\operatorname{Div}_{g, \mathbf{a}}$ is logarithmically étale over $\overline{\mathcal{M}}_{g, n}$, from which it follows that $\mathbf{D i v} g, \mathbf{a}$ has a logarithmic perfect relative obstruction theory and hence a virtual fundamental class:

Date: January 26, 2021.

2010 Mathematics Subject Classification. 14D20, 14D23, 14C17, 14 T05.

Key words and phrases. Abel-Jacobi map, logarithmic geometry, tropical geometry, moduli of curves, divisors. 
Theorem B. The Abel-Jacobi section has a logarithmic perfect relative obstruction theory whose obstruction bundle is the dual of the Hodge bundle.

Rubber targets. This work grew out of an attempt to understand the moduli space of stable maps from curves to so-called "rubber" targets and its virtual fundamental class, and generalizes our earlier work on that problem over the rational tails locus [CMW12] and compact type locus [MW13]. This space parameterizes maps from prestable curves into unparameterized rational targets. It was compactified, and equipped with a virtual fundamental class, by Graber and Vakil [GV05], based on earlier work of Li [Li01, Li02], by expanding the rational target in families.

Section 5 is devoted to comparing our approach to Graber and Vakil's. We define $\mathbf{R u b}_{g, \mathbf{a}}$ to be the subfunctor of $\mathbf{D i v}_{g, \mathbf{a}}$ parameterizing those piecewise linear functions on tropicalizations of prestable curves whose values are totally ordered in a naturally defined partial order.

Theorem C. The stack $\mathbf{R u b}_{g, \mathbf{a}}$ is a logarithmic modification of $\mathbf{D i v} \mathbf{g}_{g, \mathbf{a}}$ and its base change under the trivial section of $\mathbf{P i c}_{g, n}$ over $\overline{\mathcal{M}}_{g, n}$ is the moduli space of stable maps to rubber targets. Under this identification, the virtual fundamental classes coincide.

Both $\mathbf{R u b}_{g, \mathbf{a}}$ and $\mathbf{D i v} \mathbf{v}_{g, \mathbf{a}}$ resolve the indeterminacy of the Abel-Jacobi section, but $\mathbf{D i v}_{g, \mathbf{a}}$ is the more efficient of the two, requiring less modification of $\overline{\mathcal{M}}_{g, n}$.

The double ramification cycle. One particularly notable application of stable maps to rubber targets has been to complete the double ramification cycle. As before, let a be a set of integer weights whose sum is zero, and let $\mathrm{DR}_{g, \mathbf{a}}$ be the locus in $\mathcal{M}_{g, n}$ consisting of those $(C, \mathbf{x})$ such that $\mathcal{O}_{C}\left(\sum a_{i} x_{i}\right)$ is isomorphic to $\mathcal{O}_{C}$. In other words, $\mathrm{DR}_{g, \mathbf{a}}$ is the pullback of the zero locus $\mathcal{M}_{g, n} \rightarrow \mathbf{P i c}_{g, n}$ of $\mathbf{P i c}_{g, n}$ along the Abel-Jacobi section aj: $\mathcal{M}_{g, n} \rightarrow \mathbf{P i c}_{g, n}$.

The locus $\mathrm{DR}_{g, \mathbf{a}}$ can also be seen as the collection of marked curves $(C, \mathbf{x})$ such that there is a map $f: C \rightarrow \mathbf{P}^{1}$ with $f^{-1}[\infty]-f^{-1}[0]=\sum a_{i} x_{i}$. Its associated cycle (as a refined intersection) is therefore called the double ramification cycle.

Theorem A says that aj extends to a closed embedding $\mathbf{D i v}_{g, \mathbf{a}} \rightarrow \mathbf{P i c}_{g, n}$, and therefore gives a naturally defined extension of $\mathrm{DR}_{g, \mathbf{a}}$ to a proper morphism:

$$
\operatorname{Div}_{g, \mathbf{a}}(\mathcal{O})=\operatorname{Div}_{g, \mathbf{a}} \underset{\mathbf{P i c}_{g, n}}{\times} \overline{\mathcal{M}}_{g, n} \rightarrow \overline{\mathcal{M}}_{g, n}
$$

Theorem B gives $\operatorname{Div}_{g, \mathbf{a}}(\mathcal{O})$ a virtual fundamental class in dimension $2 g-3+n$.

This is not the only way to complete the double ramification cycle, and the original approach relies on the interpretation of $\mathrm{DR}_{g, \mathbf{a}}$ in terms of stable maps to rational curves. If $C \rightarrow \mathbf{P}^{1}$ degenerates so that a component of $C$ maps either to 0 or to $\infty$, the divisor $f^{-1}[\infty]-f^{-1}[0]$ ceases to have any meaning. To prevent this from happening, one works in $\mathbf{R u b}_{g, \mathbf{a}}$ and allows the target $\mathbf{P}^{1}$ to expand. Pulling back $\mathbf{R u b}_{g, n} \times_{\mathbf{P i c}_{g, n}} \overline{\mathcal{M}}_{g, n}$ yields a second compactification of the double ramification locus, $\mathbf{R u b}_{g, \mathbf{a}}(\mathcal{O})$, and pushing forward its virtual fundamental class gives a second completion of the double ramification cycle.

Corollary D. The two completions $\left[\operatorname{Div}_{g, \mathbf{a}}(\mathcal{O})\right]^{\mathrm{vir}}$ and $\left[\operatorname{Rub}_{g, \mathbf{a}}(\mathcal{O})\right]^{\mathrm{vir}}$ of the double ramification cycle coincide.

Since the two cycle classes coincide, we introduce $\left[\overline{\mathrm{DR}}_{g, \mathbf{a}}\right]$ for either and call it the (completed) double ramification cycle.

The double ramification cycle has received considerable attention recently, culminating in the proof of an explicit formula for $\left[\overline{\mathrm{DR}}_{g, \mathbf{a}}\right]$ by Janda, Pandharipande, Pixton, and Zvonkine [JPPZ17] (the formula was previously conjectured by Pixton [FP18]) and attendant tautological relations by Clader and Janda [CJ18]. It has also led to a search for pluricanonical variants of the double ramification cycle, completing the locus $\operatorname{DR}_{g, \mathbf{a}}\left(\omega^{\otimes k}\right)$ consisting of those marked curves $(C, \mathbf{x})$ such that $\mathcal{O}\left(\sum a_{i} x_{i}\right) \simeq \omega_{C}^{\otimes k}$. This question - which was asked us by F. Janda and R. Pandharipande - precipitated the present work.

Theorems A and B answer this question. Let $\mathbf{D i v}_{g, \mathbf{a}}\left(\omega^{\otimes k}\right)$ be the pullback of the Abel-Jacobi section along the morphism $\overline{\mathcal{M}}_{g, n} \rightarrow \mathbf{P} \mathbf{i c}_{g, n}$ that sends $(C, \mathbf{x})$ to $\omega_{C}^{\otimes k}$.

Corollary E. The projection $\operatorname{Div}_{g, \mathbf{a}}\left(\omega^{\otimes k}\right) \rightarrow \overline{\mathcal{M}}_{g, n}$ is proper, and $\mathbf{D i v}_{g, \mathbf{a}}\left(\omega^{\otimes k}\right)$ has a virtual fundamental class in dimension $2 g-3+n$. 
The same class can also be constructed using $\mathbf{R u b}_{g, \mathbf{a}}$. This facilitates a stable maps interpretation of the pluricanonical double ramification cycle, which we do not pursue here. Understanding the details of this interpretation would presumably connect our approach to Guéré's [Gué16].

Pixton's conjecture - but not its proof - was at least partly inspired by calculations of the double ramification cycle over the compact type locus due to Hain [Hai11] and Grushevsky-Zakharov [GZ12a] (in a follow up, Grushevsky and Zakharov are able to go slightly beyond compact type [GZ12b]). Those proofs rely on an extension of the Abel-Jacobi section beyond compact type, and we hope that our work might help to extend the ideas of Hain and Grushevsky-Zakharov to all of $\overline{\mathcal{M}}_{g, n}$. For the moment, however, it seems that the lack of a principal polarization on the Picard group of a curve that is not of compact type presents an additional obstacle, and that we will need to work in the logarithmic Picard group instead (see [MW18] and a forthcoming paper by Molcho, Ulirsch and Wise).

Related work. This paper replaces our preprint [MW13], which defined closely related moduli spaces but lacked the logarithmic and tropical perspectives employed here, and claimed weaker results. In the intervening years, several others have achieved similar results, also using logarithmic methods [Gué16, CC19, Hol17]. We feel that the tropical perspective we employ is natural, and likely to be useful elsewhere (see [RSPW19], for example).

Our work can also be seen as a functorial perspective on, and generlization of, the spaces of twisted canonical divisors introduced by Farkas and Pandharipande [FP18] and generalized to $k$-canonical divisors by Schmitt [Sch18].

Logarithmic geometry, tropical geometry, and their relevance to divisors. We rely heavily on logarithmic geometry as an interpolant between algebraic geometry (narrowly construed) and tropical geometry. It is a convenient language by which combinatorial data, like dual graphs of algebraic curves, can be permitted to move in algebraic families. While we summarize some of the main features of logarithmic geometry, as we use it, in Section 3, we leave the matter of a thorough introduction to others, such as Ogus's textbook [Ogu18], K. Kato's original papers [Kat89, Kat94], or the surveys $\left[\mathrm{ACG}^{+} 13, \mathrm{ACM}^{+} 16\right]$. The introduction to logarithmic structures and logarithmic curves in [CCUW20, Sections 6.1, 6.2, 7.1, and 7.2] is closely aligned with the way we use logarithmic structures here.

Within the logarithmic category, it is possible to speak of morphisms from logarithmic schemes to combinatorial fans. The details about how this works were the subject of [CCUW20, Section 6] (see also [IKNU20, Sections 2.2 and 2.3]). Perhaps surprisingly, morphisms between these combinatorial data can require meaningful geometric input, and therefore be parameterized by geometrically interesting moduli spaces.

Without trying to be too precise yet, a logarithmic structure on a scheme $C$ is a family of line bundles on $C$, indexed by a sheaf of partially ordered abelian groups $\bar{M}_{C}^{\text {gp }}$. When $C$ is a logarithmic curve over a logarithmic base $S$, we define a logarithmic divisor on $C$ to be a section of this sheaf, $\bar{M}_{C}^{g p}$ (technically, it will be an equivalence class of such sections). Our notation for the moduli space of genus $g, n$-marked logarithmic curves equipped with logarithmic divisors is $\mathbf{D i v}_{g, n}$.

The dual graph of the logarithmic curve $C$ can be given an integral piecewise linear structure (see [CCUW20, Section 7.2] for the construction) and the sheaf $\bar{M}_{C}^{\mathrm{gp}}$ can be interpreted combinatorially as the sheaf of affine, piecewise linear functions on the dual graph, with respect to this structure (see Section 3.3.3 and [CCUW20, Remark 7.3]). To give such a function requires certain relations to hold among the lengths of the edges of the dual graph, which correspond to certain identities inside $\bar{M}_{S}^{\mathrm{gp}}$, the logarithmic structure of the base.

But the logarithmic structure of $S$ also includes a family of line bundles indexed by $\bar{M}_{S}^{g p}$, and if one is to impose identities between elements of $\bar{M}_{S}^{g p}$, one must also have isomorphisms between the corresponding line bundles. The choices of these isomorphisms are parameterized by tori, which naturally appear as the interiors of the exceptional loci of the birational modification $\mathbf{D i v}_{g, n} \rightarrow \mathfrak{M}_{g, n}$ promised in Theorem C.

Once $\mathbf{D i v} \mathbf{v}_{g, n}$ has been constructed, the map to $\mathbf{P} \mathbf{i c}_{g, n}$ comes for free: a point of $\mathbf{D i v} \mathbf{v}_{g, n}$ is a logarithmic curve, equipped with a section $\alpha$ of $\bar{M}_{C}^{\mathrm{gp}}$; by definition, $\bar{M}_{C}^{\mathrm{gp}}$ parameterizes line bundles on $C$, and sending $(C, \alpha)$ to the corresponding line bundle gives the required map.

Conventions and notation. For the most part, we work in the logarithmic category, so $T_{X}$ and $\Omega_{X}$ denote the logarithmic tangent and cotangent bundles of a logarithmic scheme $X$. We write $\underline{X}$ for the underlying 
scheme of a logarithmic scheme, so $T_{\underline{X}}$ and $\Omega_{\underline{X}}$ denote the tangent and cotangent sheaves of the scheme underlying $X$.

The main exception to this convention is for moduli spaces that already have a widely recognized notation, particularly the moduli space of curves. We use $\mathcal{M}_{g, n}$ for the moduli space of smooth curves with $n$ distinct markings $(2 g-2+n>0)$, and $\overline{\mathcal{M}}_{g, n}$ for the Deligne-Mumford-Knudsen compactification. We write $\mathcal{M}_{g, n}^{\log }$ for the moduli space of logarithmic curves, whose underlying Deligne-Mumford stack is $\overline{\mathcal{M}}_{g, n}^{\log }$ [Kat00]. We use a fraktur $\mathfrak{M}$ for pre-stable variants.

Acknowledgements. This work has benefited from helpful and encouraging conversation with Gabriele Mondello and Dhruv Ranganathan. The questions and comments of Tom Graber, David Holmes, and Rahul Pandharipande have also been very helpful to us. We owe a particular debt to Felix Janda and Rahul Pandharipande for asking the questions that motivated this project. We are very grateful as well to the anonymous referees, whose careful reading and suggestions have simplified, corrected, and otherwise improved our work considerably.

J.W. was partially supported by NSA grants H98230-14-1-0107 and H98230-16-1-0329 and a Simons Collaboration Grant.

\section{The NAIVE ABel-JACOBI MAP}

We begin by describing the Abel-Jacobi section over the locus of compact type curves, in order to motivate the main construction of this paper. This section is not logically necessary for what follows, and may be omitted. In this section, we do not use any logarithmic structures.

\subsection{Compact type.}

Definition 2.1.1. Let ${ }^{c t} \operatorname{Div}_{g}$ be the space of tuples $(C, \mathbf{x}, \mathbf{a})$ consisting of a pre-stable, compact type curve $\pi: C \rightarrow S$ of genus $g$, marked sections $x_{1}, \ldots, x_{n}$ of $C$ over $S$, and a vector $\mathbf{a}=\left(a_{1}, \ldots, a_{n}\right)$ of integer weights. We decorate ${ }^{c t} \mathbf{D i v}$ with subscripts and superscripts to fix combinatorial data: ${ }^{c t} \mathbf{D i v}_{g}^{0}$ is the open and closed substack where $\sum a_{i}=0$ and ${ }^{c t} \mathbf{D i v}_{g, \mathbf{a}}$ is the open and closed substack where the vector a has been fixed.

Note that ${ }^{\text {ct }} \mathbf{D i v}_{g, \mathbf{a}}$ is isomorphic to the stack ${ }^{\text {ct }} \mathfrak{M}_{g, n}$ of $n$-marked, pre-stable, compact type curves.

Definition 2.1.2. We denote by ${ }^{c t} \mathbf{P} \mathbf{i c}_{g, n}$ the stack whose $S$-points are triples $(C, \mathbf{x}, L)$ where $(C, \mathbf{x})$ is a family of $n$-marked, pre-stable, compact type curves $C$ of genus $g$, and $L$ is a section of $\pi_{*}\left(\mathbf{B G}_{m C}\right) / \mathbf{B G}_{m S}$. We denote by ${ }^{c t} \mathbf{P} \mathbf{i c}_{g, n}^{0}$ the open substack in which $L$ has degree zero on every component of the fibers of $C$.

We construct a map ${ }^{\text {ct }} \mathbf{D i v}_{g, \mathbf{a}}^{0} \rightarrow{ }^{\text {ct }} \mathbf{P} \mathbf{i c}_{g, n}^{0}$. Suppose that $(C, \mathbf{x})$ is an $S$-point of ${ }^{\text {ct }} \mathbf{D i v} \mathbf{v}_{g, \mathbf{a}}$. We must give a line bundle on $C$, well defined up to tensoring by a line bundle pulled back from $S$. Since ${ }^{\mathrm{ct}} \mathbf{D i v}_{g, \mathbf{a}}$ is smooth, it is sufficient to treat the case where $S$ is smooth and the family $C$ is a versal deformation of its fibers. In particular, the total space of $C$ is smooth in this case.

It is also sufficient to work in an étale neighborhood of each geometric point of $S$, so we fix one and call it $s$. We may therefore assume that the boundary of $S$ is a simple normal crossings divisor and that all of its components contain $s$. Each of these components $D_{j}$ of the boundary of $S$ corresponds to an edge in the dual graph of $C_{s}$. Since $C_{s}$ is of compact type, removing this edge will disconnect the dual graph. The two components correspond locally in $S$ to divisors in the total space of $C$, so that after further étale localization around $s$, we can assume that there are divisors $C_{j}$ and $C_{j}^{\prime}$ such that $\pi^{-1} D_{j}=C_{j}+C_{j}^{\prime}$.

Now there is a choice of integers $b_{j}$ and $b_{j}^{\prime}$ such that the line bundle $L=\mathcal{O}_{C}\left(\sum a_{i} x_{i}+\sum b_{j} C_{j}+\sum b_{j}^{\prime} C_{j}^{\prime}\right)$ has degree zero on every component of $C_{s}$. The line bundle $L$ will continue to have degree zero on every component of $C_{t}$ for every $t$ in an open neighborhood of $s$, so after replacing $S$ by this open neighborhood, we obtain an object of ${ }^{\mathrm{ct}} \mathbf{P} \mathbf{i c}_{g, n}^{0}$. Moreover, the construction of $L$ is unique up to replacing $b_{j}$ and $b_{j}^{\prime}$ by $b_{j}+c$ and $b_{j}^{\prime}+c$. This has the effect of twisting $L$ by $c D_{j}$, which defines the same object of ${ }^{c t} \mathbf{P i c}_{g, n}^{0}$. It follows that our construction is independent of our choices and consistent with further localization, and therefore that it glues to a map ${ }^{\text {ct }} \mathbf{D} \mathbf{i v}_{g, \mathbf{a}}^{0} \rightarrow{ }^{\text {ct }} \mathbf{P i c} \mathbf{c}_{g, n}^{0}$.

There is a twisted variant of this construction taking values in ${ }^{c t} \mathbf{P i c}{ }_{g, n}^{k \text {-can }}$, where the superscript can refers to the open and closed substack parameterizing line bundles of the $k$-canonical multidegree $(k(2 h-2+m)$ on a component of genus $h$ with $m$ special points). Let a be a tuple of integers such that $\sum a_{i}=k(2 g-2)$. 
Then, as above, there is a unique way of twisting $\mathcal{O}_{C}\left(\sum a_{i} x_{i}\right)$ so that it has the $k$-canonical multidegree on each component. This gives a map ${ }^{\mathrm{ct}} \mathbf{D i v} \mathbf{g}_{g, \mathbf{a}}^{k \text {-can }} \rightarrow \mathbf{P i c}_{g, n}^{k \text {-can }}$.

Remark 2.1.3. We emphasize that our Abel-Jacobi map retains the configuration of the marked points, and is therefore injective on ${ }^{\mathrm{ct}} \mathbf{D i v} \mathbf{v}_{g, \mathbf{a}}$ for any fixed $\mathbf{a}$ of total weight zero. One can, of course, subsequently forget the marked points, which vary in a proper family.

Proposition 2.1.4. The map ${ }^{\mathrm{ct}} \mathbf{D i v} \mathbf{v}_{g, \mathbf{a}}^{k \text {-can }} \rightarrow{ }^{\mathrm{ct}} \mathbf{P i c}_{g, n}^{k \text {-can }}$ is a closed embedding.

Proof. The projection ${ }^{\mathrm{ct}} \mathbf{P} \mathbf{i c}_{g, n}^{k \text {-can }} \rightarrow{ }^{\mathrm{ct}} \mathcal{M}_{g, n}$ is separated and the composition ${ }^{\mathrm{ct}} \mathbf{D i v} \mathbf{v}_{g, \mathbf{a}}^{k \text {-can }} \rightarrow{ }^{\mathrm{ct}} \mathcal{M}_{g, n}$ is an isomorphism. Therefore the map ${ }^{\text {ct }} \mathbf{D i v} v_{g, \mathbf{a}}^{k \text {-can }} \rightarrow{ }^{\text {ct }} \mathbf{P i c} \mathbf{c}_{g, n}^{k \text {-can }}$ is a section of a separated map, hence a closed embedding. ${ }^{1}$

It may be important to consider multidegrees other than 0 and $2 g-2$, so we emphasize here that the same proof would show that if $L$ had a different multidegree, there would be a unique twist of $M$ by a Cartier divisor supported in the special fiber that recovers $L$. It seems difficult to formulate Proposition 2.1.4 in a global way for other multidegrees using the terminology of this section. Later on, the more general version will fall out naturally from the formulation in terms of logarithmic geometry.

Definition 2.1.5. Given a family of compact type curves $C$ over $S$ and a line bundle $L$ of multidegree 0 on $C$, let ${ }^{\text {ct }} \mathbf{D i v}_{g}^{0}(C, L)$ be the locus in ${ }^{\text {ct }} \mathbf{D i v}_{g}^{0}(C)$ where $\mathcal{O}_{C}\left(\sum a_{i} x_{i}\right) \simeq L$. More generally, if $L$ has $k$-canonical multidegree, define ${ }^{\text {ct }} \mathbf{D i v}_{g}^{k \text {-can }}(C, L)$ to be the locus in ${ }^{{ }^{c t}} \mathbf{D i v}_{g}^{k \text {-can }}(C, L)$ to be the locus in ${ }^{\text {ct }} \mathbf{D i v}{ }_{g}^{k \text {-can }}(C)$ where $\mathcal{O}_{C}\left(\sum a_{i} x_{i}\right) \simeq L$.

The following statement follows immediately from the definitions, and is included for the sake of comparison to the logarithmic results that follow.

Proposition 2.1.6. There are cartesian diagrams:
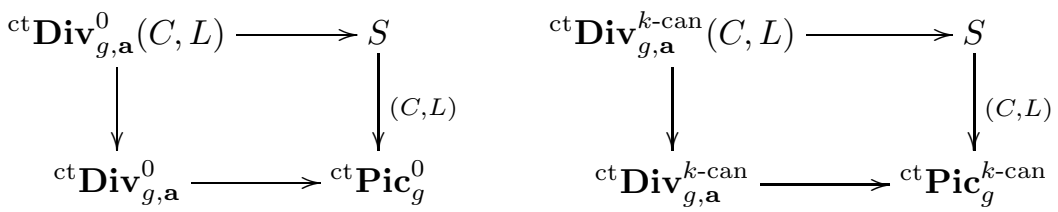

In particular, let $S={ }^{\mathrm{ct}} \mathcal{M}_{g, n}$, let $C$ be its universal curve, let $L$ be its structure sheaf, and let a be a vector of total weight zero. Then let $\mathrm{DR}_{g, \mathbf{a}}$ be the homology class of ${ }^{\mathrm{ct}} \operatorname{Div}_{g, \mathbf{a}}^{0}(C, L)$ in ${ }^{\mathrm{ct}} \mathcal{M}_{g, n}$ under the horizontal projection (which is a closed embedding). We will see later that this coincides with the double ramification cycle as defined via stable maps.

2.2. Indeterminacy of the Abel-Jacobi map. In this section we will use $\mathbf{D}_{g, \mathbf{a}}^{0}$ to denote a naïve extension of ${ }^{\mathrm{ct}} \mathbf{D i v}_{g, \mathbf{a}}^{0}$ out of the compact-type locus, which is to say the moduli space of pre-stable marked curves with integral weights summing to zero. We will see momentarily that this definition is not suitable for defining an Abel-Jacobi map, and we correct it in Section 4.

The Abel-Jacobi map becomes indeterminate outside of the compact type locus. There are two sorts of problems that can occur. The first, and perhaps more familiar, is when a divisor of total degree zero degenerates so that the degree is not zero on every component, and this defect cannot be corrected because of the topology of the dual graph and the rates of smoothing of the nodes (see Figure 1).

If $u$ and $v$ are both unit multiples of the local parameter $t$, then the total space of the family is smooth, and twisting by one of the components will change the degree on each of the two components of the special fiber by an even number. If we assign odd weights $k$ and $-k$ to $p$ and $p^{\prime}$ then, since the degree of $\mathcal{O}_{C_{0}}\left(k p-k p^{\prime}\right)$ is odd, no twist can achieve zero degree on both components. However, this is really a defect of Pic ${ }^{0}$ - the line bundle with degree zero on the general fiber has no limit on the special fiber that has degree zero on both components - and is not our concern in this paper. This defect is corrected by the logarithmic Picard group [MW18].

\footnotetext{
${ }^{1}$ We thank D. Holmes and an anonymous referee for pointing out that this simple explanation could replace our original argument. The same conclusion holds for canonical and pluricanonical variants.
} 


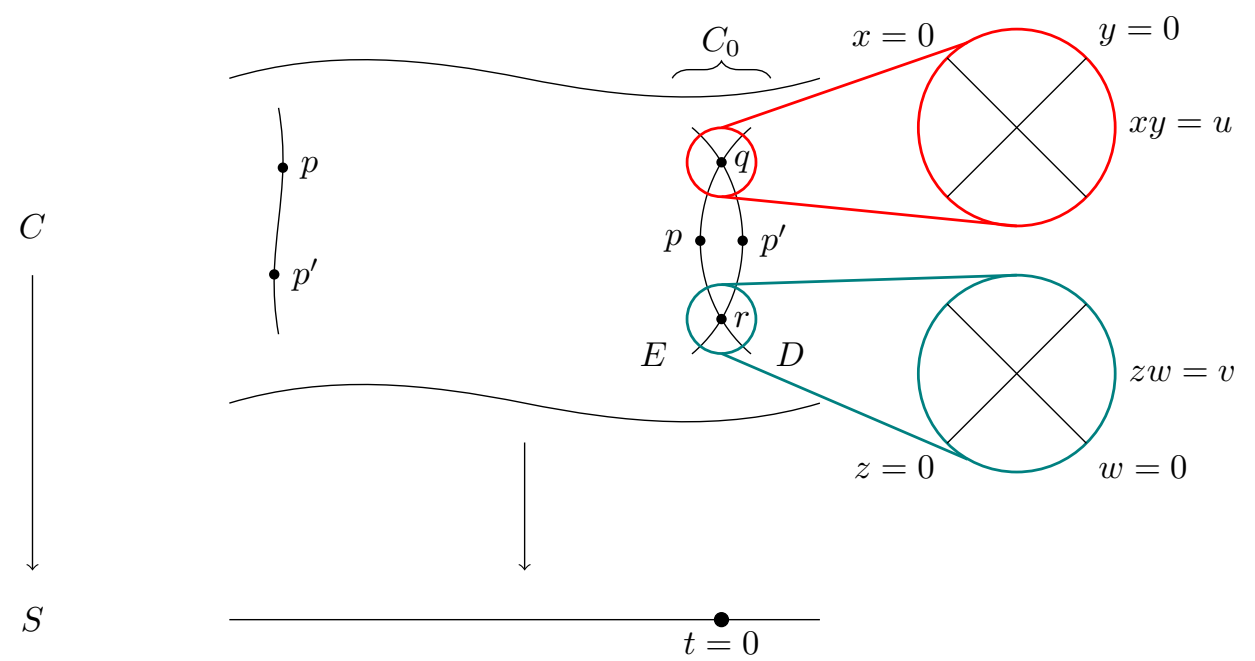

Figure 1. A family of curves deforming to a singular special fiber $C_{0}$ consisting of two components meeting at two distinct nodes.

More serious, at least for our present concerns, is the case where there is a limiting line bundle of degree zero, but this line bundle depends on the choice of the degenerating family. The divisor $k p-k p^{\prime}$ on the special fiber clearly depends only on the location of $p$ and $p^{\prime}$ on the special fiber, and not on the family, so the Abel-Jacobi section must become indeterminate at such a point. Indeed, assuming that $k=2 \ell$ is even, we can twist by $\ell D$ to get a line bundle of degree zero on the total space. However, we shall demonstrate below that if $\ell \neq 0$ then twisting by $\ell D$ changes the gluing data at the two nodes in a manner depending on the relative rates of smoothing of the two nodes. The upshot here is that we cannot have a well-defined map $\mathbf{D}_{g, \mathbf{a}}^{0} \rightarrow \mathbf{P i c}_{g, n}^{0}$ on curves of the sort illustrated in Figure 1 unless we modify $\mathbf{D}_{g, \mathbf{a}}^{0}$ to include additional data that can resolve the indeterminacy.

We explain precisely how this indeterminacy arises in the example above (see Figure 1 for notation). It is interesting to consider a general situation where $u$ and $v$ are not necessarily unit multiples of the smoothing parameter, but where there are nonzero integers $a$ and $b$ such that the ratio of $u^{a}$ and $v^{b}$ is a unit, and $a+b=k$. Of course, $a$ and $b$ must have the same sign if this is to happen.

Under these conditions, we can describe a Cartier divisor $E^{\prime}$ supported on $E$ by the following local equations:

$$
\left\{\begin{array}{l}
x^{a}=0 \text { near } q \\
z^{b}=0 \text { near } r \\
u^{a}=v^{b}=0 \text { near } D, \text { away from } q \text { and } r \\
1=0 \text { elsewhere }
\end{array}\right.
$$

This divisor has numerical intersection $a+b=k$ with $D$. A similar construction gives a divisor $D^{\prime}$ having numerical intersection $k$ with $E$. Then $D^{\prime}+E^{\prime}$ is a multiple of a fiber, hence is trivial, and $D^{\prime} \cdot D=E^{\prime} \cdot E=$ $-k$. We note that $\mathcal{O}_{C}\left(D^{\prime}+E^{\prime}\right)$ is not canonically trivial, but rather is trivialized by the choice of some $\alpha u^{-a}=\beta v^{-b}$. We fix one such trivialization.

Now take $L=\mathcal{O}_{C}\left(k p-k p^{\prime}\right)$. A line bundle on $C_{0}$ can be specified by giving a line bundle $L_{D}^{\prime}$ on $D$, a line bundle $L_{E}^{\prime}$ on $E$, and isomorphisms $\left.\left.L_{D}^{\prime}\right|_{q} \simeq L_{E}^{\prime}\right|_{q}$ and $\left.\left.L_{D}^{\prime}\right|_{r} \simeq L_{E}^{\prime}\right|_{r}$. We work out these gluing isomorphisms when $L^{\prime}=L\left(D^{\prime}\right)$.

Set $L_{D}=\left.L\right|_{D}$ and $L_{E}=\left.L\right|_{E}$. We certainly have $\left.L_{D}\right|_{q}=\left.L_{E}\right|_{q}$ and $\left.L_{D}\right|_{r}=\left.L_{E}\right|_{r}$. We set $L_{D}^{\prime}=$ $L_{D}(-a q-b r)$ and $L_{E}^{\prime}=L_{E}(a q+b r)$. The gluing isomorphism is:

$$
\left.L_{D}^{\prime}\right|_{q}=\left.L_{D}(-a q)\right|_{q}=\left.\left.L\left(-E^{\prime}\right)\right|_{q} \underset{\alpha u^{-a}}{\sim} L\left(D^{\prime}\right)\right|_{q}=\left.L_{E}(a q)\right|_{q}=\left.L_{E}^{\prime}\right|_{q}
$$


A local generator $x^{a}$ for $\left.L_{D}^{\prime}\right|_{q}$ is carried under this isomorphism to $\alpha u^{-a} x^{a}=\alpha y^{a}$ since $x y=u$ near $q$. Therefore changing the smoothing parameter $u$ at $q$ by a unit $\lambda$ has the effect of scaling the gluing parameter by $\lambda^{-a}$.

A similar calculation near $r$ shows that adjusting $v$ by a unit $\mu$ has the effect of scaling the gluing parameter of $L^{\prime}$ at $r$ by $\mu^{-b}$. If $u$ and $v$ are scaled by units so that $u^{a} / v^{b}$ is changed, then so will be the limiting line bundle $L^{\prime}$ in $\mathbf{P i c}{ }^{0}$ determined by this family. In particular, if $k \neq 0$, then $L^{\prime}$ depends on the relative rates of smoothing of the nodes $q$ and $r$.

This calculation also illustrates how to resolve the indeterminacy, at least in this example: we should blow up $\mathbf{D}_{g, \mathbf{a}}$ at the intersection of the boundary divisors corresponding to $Q$ and $R$ so as to introduce a coordinate that keeps track of the ratio between $u^{a}$ and $v^{b}$.

Now let us consider a 2-parameter base, with local coordinates $u$ and $v$. Then $\mathcal{O}_{C}\left(k p-k p^{\prime}\right)$ is a section of $\mathbf{P i c}^{0}(C)$ over the complement of the special point of $S$. Let $Z$ be its closure. We will see in Section 4.4 that our main theorem implies that $Z$ is not normal if $D$ and $E$ have genus 0 and $k>2$. As a sanity check, we verify this directly.

We have just seen that the special fiber $Z_{0}$ of $Z$ is all of $\mathbf{P i c}^{0}\left(C_{0}\right) \simeq \mathbf{G}_{m}$. For any nonzero integers $a$ and $b$ of the same sign such that $a+b=k$, we can find a discrete valuation $\gamma$ of the residue field at the generic point of $S$ such that $\gamma\left(u^{a}\right)=\gamma\left(v^{b}\right)$. Let $T_{a, b}$ be the spectrum of this valuation ring.

By the discussion above, the restriction $Z_{a, b}$ of $Z$ to $T_{a, b}$ has a limit in $Z$, which must be the generic point of $Z_{0}$. Each choice of $a$ and $b$ as above therefore gives a distinct valuation of the local ring of $Z$ at the generic point of $Z_{0}$. Since $k>2$, there is more than one choice for $a$ and $b$, which implies that the local ring at $Z_{0}$ is a 1-dimensional, noetherian, local ring but not a discrete valuation ring, hence is not integrally closed.

\section{REVIEW OF LOGARITHMIC GEOMETRY}

3.1. Logarithmic structures. We briefly recall the needed definitions from logarithmic geometry and refer the reader to [Kat89] or [Ogu18] for a more thorough introduction.

Recall that a monoid is a commutative semigroup with a unit and a homomorphism of monoids is required to preserve the unit element. Given a monoid $M$ we denote by $M^{\text {gp }}$ its groupification defined by the universal property that any morphism from $M$ to an abelian group factors through $M^{\text {gp }}$ uniquely. A monoid $M$ is called integral if the universal map $M \rightarrow M^{\text {gp }}$ is injective. All monoids in this paper will be integral.

It is important to note that an integral monoid $M$ can be seen as the set of elements $\geq 0$ in a partial semiorder on the associated group $M^{\mathrm{gp}}$. If the monoid is sharp, meaning its only invertible element is the identity, then the partial semiorder is a partial order. We will use this perspective frequently, particularly in Section 5.3.

A monoid is called fine if it is integral and finitely generated. An integral monoid is called saturated if $m \in M^{\mathrm{gp}}$ and $n m \in M$ for some positive integer $n$ implies $m \in M$. We apply the same adjectives for sheaves of monoids and we often write simply monoid in place of "sheaf of monoids".

A logarithmic structure on a scheme $S$ is an étale sheaf of monoids $M_{S}$ and a homomorphism $\varepsilon: M_{S} \rightarrow \mathcal{O}_{S}$ where the target is given its multiplicative monoid structure and such that every unit of $\mathcal{O}_{S}$ has a unique preimage under $\varepsilon$. In other words, $\varepsilon$ restricts to a bijection $\varepsilon^{-1}\left(\mathcal{O}_{S}^{*}\right) \rightarrow \mathcal{O}_{S}^{*}$. We therefore use $\varepsilon^{-1}$ to denote the composition $\mathcal{O}_{S}^{*} \rightarrow M_{S} \rightarrow M_{S}^{\mathrm{gp}}$. The monoid structure on $M_{S}$ will be written multiplicatively. A morphism $M_{S} \rightarrow N_{S}$ of logarithmic structures on $S$ is a morphism of sheaves of monoids compatible with their morphisms to $\mathcal{O}_{S}$.

We define $\bar{M}_{S}=M_{S} / \varepsilon^{-1}\left(\mathcal{O}_{S}^{*}\right)$ and call it the characteristic sheaf of monoids or simply the characteristic monoid of the logarithmic structure. We notate the monoid structure of $\bar{M}_{S}$ additively. By construction, $\bar{M}_{S}$ is sharp, so the induced semiorder on $\bar{M}_{S}^{\mathrm{gp}}$ is a partial order.

By definition, there is an exact sequence of étale sheaves of abelian groups

$$
0 \rightarrow \mathcal{O}_{S}^{*} \stackrel{\varepsilon^{-1}}{\longrightarrow} M_{S}^{\mathrm{gp}} \rightarrow \bar{M}_{S}^{\mathrm{gp}} \rightarrow 0
$$

which induces a map

$$
\Gamma\left(S, \bar{M}_{S}^{\mathrm{gp}}\right) \rightarrow H^{1}\left(S, \mathcal{O}_{S}^{*}\right)
$$

that will play a crucial role in this paper. That is, every section $\alpha$ of the characteristic monoid $\bar{M}_{S}^{\text {gp }}$ induces a $\mathcal{O}_{S}^{*}$-torsor $\mathcal{O}_{S}^{*}(-\alpha)$ on $S$. In concrete terms, $\mathcal{O}_{S}^{*}(-\alpha)$ is the coset of $\varepsilon^{-1} \mathcal{O}_{S}^{*}$ in $M_{S}^{\text {gp }}$ classified by $\alpha$. The 
restriction of $\varepsilon$ to $\mathcal{O}_{S}^{*}(-\alpha)$ induces a homomorphism of line bundles:

$$
\varepsilon_{\alpha}: \mathcal{O}_{S}(-\alpha) \rightarrow \mathcal{O}_{S}
$$

We also use $\varepsilon_{\alpha}$ to denote the dual map $\mathcal{O}_{S} \rightarrow \mathcal{O}_{S}(\alpha)$. This construction of the line bundle $\mathcal{O}_{S}(\alpha)$ will be used throughout the remainder of the paper.

Logarithmic schemes will be defined to be schemes equipped with logarithmic structures that possess local charts. We prefer a slightly more permissive definition of a chart, and therefore of quasicoherence, than does Kato [Kat89, Section 2.1]:

Definition 3.1.1. A chart for a logarithmic structure $M$ on $X$ is a monoid $P$ and a homomorphism $P \rightarrow \Gamma\left(X, \bar{M}_{X}\right)$ such that $M_{X}$ is the initial example of a logarithmic structure on $X$ admitting such a map. A logarithmic structure is called quasicoherent if it has charts étale-locally, and is called coherent if it is quasicoherent and the monoids $P$ can be chosen finitely generated.

Preferences aside, the difference between Kato's definition of quasicoherence and ours has no logical bearing on this paper. Our charts are equivalent to Kato's when $P$ is finitely generated, and therefore our coherent logarithmic structures coincide with Kato's, although our quasicoherent logarithmic structures are more inclusive. Almost all logarithmic structures in this paper will be quasicoherent, and in fact coherent. The only place where we make use of non-coherent logarithmic structures is in the valuative criterion for properness, proved in Section 3.5, but the notion of quasicoherence does not intervene there.

Example 3.1.2. Suppose that $X$ is a smooth curve of genus 1 and $A \subset X$ is a set of closed points. Each finite subset of $A$ generates a divisorial logarithmic structure on $X$. The union of these logarithmic structures, under the natural inclusion maps, is a quasicoherent logarithmic structure in our sense, but if $A$ is not contained in a finitely generated subgroup of $X$ then this logarithmic structure is not quasicoherent in Kato's sense.

We will use the term logarithmic scheme to mean a scheme $S$ equipped with a logarithmic structure $\varepsilon: M_{S} \rightarrow \mathcal{O}_{S}$ that is quasicoherent and integral. If $S$ and $T$ are logarithmic schemes equipped with logarithmic structures and $f: S \rightarrow T$ is a morphism of schemes, there is a univeral (initial) way of extending the composition $f^{-1} M_{T} \rightarrow f^{-1} \mathcal{O}_{T} \rightarrow \mathcal{O}_{S}$ to a logarithmic structure $f^{*} M_{T}$ on $S$. The characteristic monoid of $f^{*} M_{T}$ coincides with the étale pullback of sheaves $f^{-1} \bar{M}_{T}$. A morphism of logarithmic schemes from $\left(S, M_{S}\right)$ to $\left(T, M_{T}\right)$ is a morphism of schemes $f: S \rightarrow T$ and a morphism of logarithmic structures $f^{*} M_{T} \rightarrow M_{S}$.

Morphisms of logarithmic schemes are logarithmically smooth (resp. étale) if they are locally of finite presentation on the underlying schemes, their logarithmic structures are also locally finitely presented, and they satisfy the right lifting property (resp. the unique right lifting property) for strict infinitesimal extensions of logarithmic schemes. See [Kat89, Section 3] for a thorough introduction to logarithmic differentials and smoothness.

Logarithmic structures will not be assumed saturated, although a saturation hypothesis may usually be imposed with no significant change to the results. The exception to this rule is Section 5, where the comparison to Graber and Vakil's moduli space is applies only to the non-saturated variants.

3.2. Logarithmic curves. We recall the local description of a logarithmic curve. We refer the reader to [Kat00] for further background.

Definition 3.2.1. Let $S$ be a logarithmic scheme. A logarithmic curve over $S$ is a morphism $\pi: C \rightarrow S$ of logarithmic schemes that is logarithmically smooth with connected geometric fibers and has the following local description at every geometric point $x$ of $C$ over a geometric point $s$ of $S$ : either

(i) $x$ is a smooth point of the underlying curve of $C$ and $\bar{M}_{C, x} \simeq \bar{M}_{S, s}$, or

(ii) $x$ is a smooth point of the underlying curve of $C$ and $\bar{M}_{C, x} \simeq \bar{M}_{S, s}+\mathbf{N}$, with the additional generator corresponding to a local parameter of $C$ at $x$, or

(iii) $x$ is a node of the underlying curve of $C$ and $\bar{M}_{C, x} \simeq \bar{M}_{S, s}+\mathbf{N} \alpha+\mathbf{N} \beta /(\alpha+\beta=\delta)$ for some $\delta \in \bar{M}_{S, s}$, with $\alpha$ and $\beta$ corresponding to the parameters for $C$ along its branches at $x$ and $\delta$ corresponding to a parameter on the base smoothing the node. 
In the third part, the element $\delta \in \bar{M}_{S, s}$ is known as the smoothing parameter of the node. An alternative local description to (iii) is

$$
\bar{M}_{C, x} \simeq\left\{(\rho, \sigma) \in \bar{M}_{S, s} \times \bar{M}_{S, s} \mid \rho-\sigma \in \mathbf{Z} \delta\right\} .
$$

These two descriptions may be identified by sending $\gamma+a \alpha+b \beta$ to $(\gamma+a \delta, \gamma+b \delta)$.

Logarithmic curves can be defined more parsimoniously as logarithmically smooth, integral, saturated families with connected fibers (see [Kat00, Definition 1.1 and Theorem 1.1] and [Tsu97, Theorem 4.2]). The local description will be more important in this paper.

3.3. Tropical curves. Suppose that $S$ is a logarithmic point whose underlying scheme is the spectrum of an algebraically closed field and $C$ is a proper logarithmic curve over $S$. The tropicalization of $C$ is the dual graph of $C$, with a leg for each marked point and each edge metrized by its smoothing parameter (Definition 3.2.1).

Remark 3.3.1. The smoothing parameters live inside the characteristic monoid $\bar{M}_{S}$ of the base, which is not necessarily equal to $\mathbf{R}_{\geq 0}$, so we are not endowing the dual graph with a metric in the usual sense. However, each homomorphism $\bar{M}_{S} \rightarrow \mathbf{R}_{\geq 0}$ induces a metric on the dual graph, by applying the homomorphism to the smoothing parameters, so what we have is actually a family of metrics, parameterized by $\operatorname{Hom}\left(\bar{M}_{S}, \mathbf{R}_{\geq 0}\right)$. We therefore ask the reader to indulge us in our abuse of terminology, and permit us to call this labelling a metric.

Remark 3.3.2. It is possible to make sense of a varying family of tropical curves over a logarithmic base scheme [CCUW20, Section 7], but we will not need to do so here.

Let $\Gamma_{C}$ be the tropicalization of $C$. Following [CCUW20] we understand sections of $\bar{M}_{C}^{\mathrm{gp}}$ through their correspondence to piecewise linear functions on $\Gamma$ valued in $\bar{M}_{S}^{\mathrm{gp}}$ and linear with integer slopes along the edges of $\Gamma$. We summarize this correspondence below, with further details to be found in [CCUW20, Remark 7.3].

3.3.3. Piecewise linear functions. Suppose that the underlying scheme of $S$ is the spectrum of an algebraically closed field, that $C$ is a logarithmic curve over $S$, and that $\alpha$ is a section of $\bar{M}_{S}^{\mathrm{gp}}$. As $\bar{M}_{C}^{\mathrm{gp}}$ is constructible, a section can be described by giving a value in the stalk of $\bar{M}_{C}$ at a geometric generic point of each stratum, in such that these values are compatible with geometric specialization. The open strata correspond to the vertices of the dual graph of $C$, and we have $\bar{M}_{C, x}^{\mathrm{gp}}=\bar{M}_{S}^{\mathrm{gp}}$ at such a point. Therefore $\alpha$ induces $\alpha(v) \in \bar{M}_{S}^{\mathrm{gp}}$ for each vertex $v$ of the dual graph of $C$.

These values are not arbitrary, but are constrained by the values at the nodes. Suppose that $x$ is a node of $C$ generizing to geometric generic points $y$ and $z$. (If $x$ connected a component of $C$ to itself then $y=z$ but the generization maps are distinct.) This induces a map

$$
\bar{M}_{C, x}^{\mathrm{gp}} \rightarrow \bar{M}_{C, y}^{\mathrm{gp}} \times \bar{M}_{C, z}^{\mathrm{gp}} \simeq \bar{M}_{S}^{\mathrm{gp}} \times \bar{M}_{S}^{\mathrm{gp}}
$$

that is injective and furnishes an identification

$$
\bar{M}_{C, x}^{\mathrm{gp}} \simeq\left\{(a, b) \in \bar{M}_{S}^{\mathrm{gp}} \times \bar{M}_{S}^{\mathrm{gp}} \mid b-a \in \mathbf{Z} \delta\right\}
$$

where $\delta \in \bar{M}_{S}$ is the smoothing parameter of the node $x$. In the special case where $\bar{M}_{S}=\mathbf{R}_{\geq 0}$, we can recognize this characterization of $\bar{M}_{C, x}^{\mathrm{g} p}$ as the set of affine linear functions on an interval of length $\delta$ having integer slope.

Applying this observation to every edge of the dual graph of $C$, we see that, if $\bar{M}_{S}=\mathbf{R}_{\geq 0}$, a section of $\bar{M}_{C}^{\mathrm{gp}}$ amounts to a piecewise linear function on the dual graph of $C$ that is linear with integer slope along the edges and takes values in $\mathbf{R}$. When $\bar{M}_{S}$ is not $\mathbf{R}_{\geq 0}$, this interpretation ceases to make literal sense, but we continue to employ the same terminology and regard $\alpha \in \Gamma\left(C, \bar{M}_{C}^{\mathrm{gp}}\right)$ as a piecewise linear function on the dual graph of $C$, taking values in $\bar{M}_{S}^{\mathrm{gp}}$. As in Remark 3.3.1, this is not merely an analogy, since each homomorphism $\bar{M}_{S} \rightarrow \mathbf{R}_{\geq 0}$ induces a piecewise linear function on the graph, as described above, and therefore $\alpha$ may be regarded as a family of piecewise linear functions, parameterized by $\operatorname{Hom}\left(\bar{M}_{S}, \mathbf{R}_{\geq 0}\right)$. 
3.3.4. Line bundles on logarithmic curves. Let $S$ be a logarithmic scheme whose underlying scheme is the spectrum of an algebraically closed field. As we have seen above, (piecewise linear) maps from a logarithmic curve $C$ over $S$ into $\mathbf{G}_{m}^{\text {trop }}$ correspond to piecewise linear functions on the tropicalization, valued in $\bar{M}_{S}$. By way of the coboundary map,

$$
H^{0}\left(C, \bar{M}_{C}^{\mathrm{gp}}\right) \rightarrow H^{1}\left(C, \mathcal{O}_{C}^{*}\right)
$$

any such function $\alpha$ induces a line bundle $\mathcal{O}_{C}(-\alpha)$ with associated $\mathcal{O}_{C}^{*}$-torsor $\mathcal{O}_{C}^{*}(-\alpha)$. Let $C_{v}$ be the irreducible component of $C$ corresponding to a vertex $v$ of the tropicalization of $C$. We calculate the restriction, $\mathcal{O}_{C_{v}}(-\alpha)$, of $\mathcal{O}_{C}(-\alpha)$ to $C_{v}$.

Replacing $\alpha$ by $\alpha-\alpha(v)$ will change $\mathcal{O}_{C_{v}}(-\alpha)$ by $\mathcal{O}_{C_{v}}(\alpha(v))$, which is pulled back from $S$ because $\alpha(v) \in \bar{M}_{S}^{\mathrm{gp}}$. It will therefore suffice to compute $\mathcal{O}_{C_{v}}(-\alpha)$ under the assumption that $\alpha(v)=0$.

Consider a node or marked point $p$ of $C_{v}$ along which $\alpha$ has negative slope. Let $x \in M_{C_{v}, p}$ be a local coordinate of $C_{v}$ at $p$ with image $\bar{x}$ in $\bar{M}_{C_{v}, p}$. Then $\bar{M}_{C_{v}, p}^{g p}=\bar{M}_{S}^{g p}+\mathbf{Z} \bar{x}$. As $\alpha(v)=0$, we have $\alpha_{p}=\lambda \bar{x}$ for some $\lambda \in \mathbf{Z}$ - the slope of $\alpha$ along the edge corresponding to $p$.

By definition, $\mathcal{O}_{C_{v}}^{*}(-\alpha)$ is the $\mathcal{O}_{C_{v}}^{*}$-torsor of sections of $M_{C}^{\text {gp }}$ projecting to $\alpha$ in $\bar{M}_{C}^{\text {gp }}$. This means we may identify $\mathcal{O}_{C_{v}}^{*}(-\alpha)$ canonically, in a neighborhood of $p$, with $x^{\lambda} \mathcal{O}_{C_{v}}^{*}=\mathcal{O}_{C_{v}}^{*}(-\lambda p)$.

These observations demonstrate the following proposition:

Proposition 3.3.3. Let $C$ be a logarithmic curve over $S$, where the underlying scheme of $S$ is the spectrum of an algebraically closed field. Let $\alpha$ be a piecewise linear function on the tropicalization of $C$. Then, for each vertex $v$ of the tropicalization of $C$, we have a canonical identification:

$$
\mathcal{O}_{C_{v}}(-\alpha)=\mathcal{O}_{S}(-\alpha(v)) \otimes \mathcal{O}_{C_{v}}\left(-\sum \lambda_{p} p\right)
$$

The sum is taken over nodes and marked points of $C$ lying on $C_{v}$ and $\lambda_{p}$ is the slope of $\alpha$ along the edge or leg of the dual graph of $C$ corresponding to $p$, oriented away from $v$.

3.4. Logarithmic modifications. For us, a toric variety is a normal, equivariant partial compactification of an algebraic torus, with logarithmic structure associated to the inclusion of the dense torus. Suppose that $\Sigma$ is a rational polyhedral fan in a real vector space with integral lattice $L$. For any logarithmic scheme $Z$, define $Y_{\Sigma}(Z)$ to be the set of $\alpha \in \operatorname{Hom}\left(L, \Gamma\left(Z, M_{Z}^{\mathrm{gp}}\right)\right.$ such that, locally in $Z$, there is a choice of $\sigma \in \Sigma$ such that $\alpha(\sigma) \subset \Gamma\left(Z, M_{Z}\right)$. Then $Y_{\Sigma}$ is representable by a toric variety, and every toric variety arises this way. If $\Sigma^{\prime}$ is a subdivision of $\Sigma$ then $Y_{\Sigma^{\prime}} \rightarrow Y_{\Sigma}$ is a birational modification.

Definition 3.4.1. Let $X$ be a logarithmic scheme. A morphism of logarithmic schemes $X^{\prime} \rightarrow X$ that is locally pulled back from a toric modification of toric varieties is called a logarithmic modification.

Proposition 3.4.2. Logarithmic modifications are proper, logarithmically étale monomorphisms. Logarithmic modifications of logarithmically regular ${ }^{2}$ logarithmic schemes are also birational.

Proof. In a logarithmically regular logarithmic scheme, the locus where the logarithmic structure is trivial is a dense open subset. This follows from [Kat94, Theorem (3.1)]. There are no nontrivial logarithmic modifications of a logarithmic scheme with trivial logarithmic structure, so it follows as well that logarithmic modifications of logarithmically regular logarithmic schemes are birational.

The remaining assertions are all local on the base and stable under base change, so it suffices to prove them for an affine toric variety with its toric logarithmic structure. Suppose $X$ is the affine toric variety corresponding to the rational polyhedral cone $\tau$. Then the toric modifications $X^{\prime} \rightarrow X$ correspond to the subdivisions of $\tau$ into fans $\Sigma$. We can describe the functor of points of $X^{\prime}$ directly in terms of this subdivision: a map $S \rightarrow X^{\prime}$ is a map $S \rightarrow X$ such that $\tau^{\vee} \rightarrow \Gamma\left(S, \bar{M}_{S}\right)$ factors through $\sigma^{\vee}$ for some $\sigma \in \Sigma$.

We observe that the functor of points of $X^{\prime}$ is defined by a condition (as opposed to an additional datum) relative to that of $X$, which shows that logarithmic modifications are monomorphisms. Since this condition can be checked on the level of characteristic monoids, it is automatically logarithmically étale. Properness is well-known from the theory of toric varieties.

\footnotetext{
${ }^{2}$ The definition of logarithmic regularity in [Kat94, Definition 2.1] requires Zariski-local charts, but is an étale-local property by [Kat94, Theorem 3.1]. There it is reasonable to apply the term 'logarithmically regular' to logarithmic schemes that only have charts étale-locally.
} 
3.5. The valuative criterion. Recall that the pullback of logarithmic structures has a right adjoint [Kat89, Section 1.4], called pushforward, but that the pushforward of a quasicoherent logarithmic structure is not necessarily quasicoherent. If $f: X \rightarrow Y$ is a morphism of logarithmic schemes and $M_{X}$ is a logarithmic struture on $X$ then the pushforward logarithmic structure is $\mathcal{O}_{X} \times_{f_{*}} \mathcal{O}_{Y} f_{*} M_{X}$.

Definition 3.5.1. Let $j: \eta \rightarrow S$ be the inclusion of the generic point in the spectrum of a valuation ring. If $M_{\eta}$ is a logarithmic structure on $\eta$, we refer to the pushforward logarithmic structure $\mathcal{O}_{S} \times_{j_{*} \mathcal{O}_{\eta}} j_{*} M_{\eta}$ as the maximal extension of the logarithmic structure of $\eta$.

We again caution that the maximal extension $M_{S}$ of the logarithmic structure of $\eta$ may fail to be quasicoherent. This will not be a problem, since we will only be interested in maps out of $S$ with its maximal logarithmic structure, and one can make sense of maps into a logarithmic scheme, or moduli problem over logarithmic schemes, from an arbitrary locally ringed space with logarithmic structure.

Theorem 3.5.2. Let $f: X \rightarrow Y$ be a morphism of logarithmic schemes. The morphism of schemes $\underline{f}: \underline{X} \rightarrow \underline{Y}$ underlying $f$ satisfies the valuative criterion for properness if and only if $f$ has the right lifting property with respect to the inclusion of the generic point in the spectrum of a valuation ring with maximal logarithmic structure:

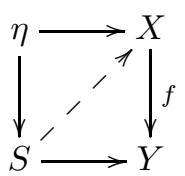

The valuative criterion also applies if we assume that the generic logarithmic structure of $S$ is valuative.

Proof. Consider a lifting problem (3.2):

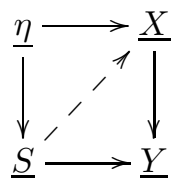

Let $M_{\eta}$ be the logarithmic structure on $\eta$ pulled back from $X$ and let $M_{S}^{\prime}$ be the logarithmic structure on $\underline{S}$ pulled back from $Y$. Let $M_{S}$ be the maximal extension of $M_{\eta}$ to $S$. Let $N_{\eta}$ be an extension of the logarithmic structure $M_{\eta}$, and let $N_{S}$ be the maximal extension of $N_{\eta}$ to $S$. By definition, we have $M_{S}=N_{S} \times_{j_{*}} N_{\eta} j_{*} M_{\eta}$.

The commutative square (3.2) gives a morphism $M_{\eta}^{\prime} \rightarrow M_{\eta}$, from which we obtain $M_{S}^{\prime} \rightarrow M_{S}$ by adjunction. We then have a commutative diagram (3.3) of solid arrows:

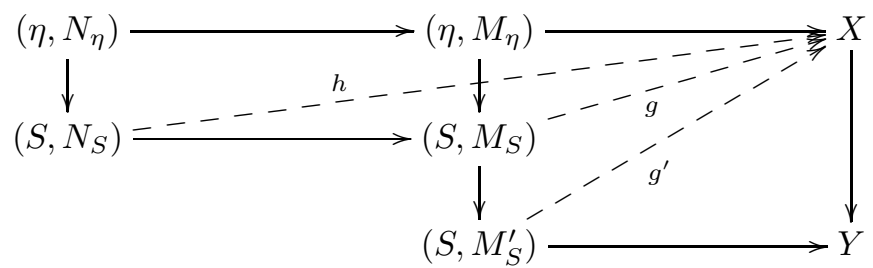

Lifting diagram (3.2) in the category of schemes is equivalent to finding a lifting $h$ in diagram (3.3). We claim that to produce any of the three dashed arrows in diagram (3.3) is equivalent. Indeed, $h$ determines $g$ and $g^{\prime}$ by composition. But $h$ determines $g$ by the observation that $M_{S}=N_{S} \times_{j_{*} N_{\eta}} j_{*} M_{\eta}$. Likewise, $g$ determines $g^{\prime}$ by the universal property of the pulled back logarithmic structure $M_{S}^{\prime}$.

Since liftings $g^{\prime}$ induce liftings $h$, we conclude that if $\underline{X} \rightarrow \underline{Y}$ satisfies the valuative criterion for properness then (3.1) has a lift whenever the logarithmic structure on $S$ is the maximal extension of a logarithmic structure on $\eta$. Conversely if we have lifting whenever the logarithmic structure is valuative then we can choose $N_{S}$ above to be valuative (since there is always a map from the logarithmic structure $M_{\eta}$ to a valuative one) and then we obtain a lift of (3.2), proving that $\underline{X} \rightarrow \underline{Y}$ satisfies the valuative criterion for properness.

While it would be possible to formulate a version of this criterion that involves only quasicoherent logarithmic strutures, this will not be necessary for our applications. 
Definition 3.5.3. We say that a homomorphism of integral monoids $f: \bar{M} \rightarrow \bar{N}$ is relatively valuative if, for every $x \in \bar{M}^{\mathrm{gp}}$, if $f(x)=0$, then either $x \in \bar{M}$ or $-x \in \bar{M}$.

Remark 3.5.4. The terminology is motivated from the case where $\bar{N}=0$, in which the condition says that every $x \in \bar{M}^{\mathrm{gp}}$ or its inverse appears in $\bar{M}$. These are the sorts of monoids that appear as the non-negative elements in the value groups of valuation rings.

Proposition 3.5.5. Suppose that $S$ is the spectrum of a valuation ring, with the maximal logarithmic structure $M_{S}$ extending a logarithmic structure at its generic point. Let $j: \eta \rightarrow S$ denote the inclusion of the generic point. Then the generization map $\bar{M}_{S} \rightarrow j_{*} \bar{M}_{\eta}$ is relatively valuative.

Proof. We observe that, by the construction of $M_{S}=\mathcal{O}_{S} \times_{j_{*} \mathcal{O}_{\eta}} j_{*} \bar{M}_{\eta}$, the kernel of $\bar{M}_{S}^{\mathrm{gp}} \rightarrow j_{*} \bar{M}_{\eta}^{\mathrm{gp}}$ is the associated group of the monoid

$$
\left(\mathcal{O}_{S} \underset{j_{*}}{\times} \mathcal{O}_{\eta} j_{*} \mathcal{O}_{\eta}^{*}\right) / \mathcal{O}_{S}^{*}
$$

which is simply the valuation monoid of $S$. It follows immediately that the generization map is relatively valuative.

3.6. Logarithmic structures of curves in degenerating families. Proposition 3.6.4 is technical, but important in our proof that the Abel-Jacobi map satisfies the valuative criterion for properness (Lemma 4.3.3.4). We begin with a local cohomology calculation that will be used in the proof of Proposition 3.6.4. The reader who prefers to do so may omit these preliminaries and rely instead on [Hol19, Theorem 4.1] for the same result.

Lemma 3.6.1. Suppose that $A$ is a ring and $t \in A$ is not a zero divisor. Let $C=\operatorname{Spec} A[x, y] /(x y-t)$, let $i: Z \subset C$ be the inclusion of the vanishing locus of $x$ and $y$, and let $U \subset C$ be its complement. Then $\mathcal{O}_{C} \rightarrow j_{*} \mathcal{O}_{U}$ is an isomorphism.

Proof. For a quasicoherent sheaf $F$ on $C$ and a closed subset $Z$ of $C$, let $\mathcal{H}_{Z}^{0}(F)$ denote the subsheaf of sections of $F$ with support in $Z$. We have an exact sequence [Gro05, Corollaire I.2.11]:

$$
0 \rightarrow \mathcal{H}_{Z}^{0}\left(\mathcal{O}_{C}\right) \rightarrow \mathcal{O}_{C} \rightarrow \mathcal{O}_{C-Z} \rightarrow \mathcal{H}_{Z}^{1}\left(\mathcal{O}_{C}\right) \rightarrow 0
$$

We must show that $\mathcal{H}_{Z}^{0}\left(\mathcal{O}_{C}\right)=\mathcal{H}_{Z}^{1}\left(\mathcal{O}_{C}\right)=0$. The vanishing of $\mathcal{H}_{Z}^{0}\left(\mathcal{O}_{C}\right)$ is clear, because $\mathcal{O}_{C}$ has no nonzero torsion. Let $Y$ be the vanishing locus of $y$ in $C$. As $\mathcal{H}_{Z}^{0}$ has an exact left adjoint, we have a spectral sequence $\mathcal{H}_{Z}^{p} \mathcal{H}_{Y}^{q}\left(\mathcal{O}_{C}\right) \Rightarrow \mathcal{H}_{Z}^{p+q}\left(\mathcal{O}_{C}\right)$. It will therefore suffice to show

$$
\mathcal{H}_{Z}^{0} \mathcal{H}_{Y}^{1}\left(\mathcal{O}_{C}\right)=0 \quad \text { and } \quad \mathcal{H}_{Z}^{1} \mathcal{H}_{Y}^{0}\left(\mathcal{O}_{C}\right)=0
$$

As $y$ is not a zero divisor in $A[x, y] /(x y-t)$, we have $\mathcal{H}_{Y}^{0}\left(\mathcal{O}_{C}\right)=0$, which gives the latter vanishing above. We compute $\mathcal{H}_{Y}^{1}\left(\mathcal{O}_{C}\right)$ with the following exact sequence (using the quasicoherence of the local cohomology sheaves [Gro05, Corollaire II.4]):

$$
0 \rightarrow A[x, y] /(x y-t) \rightarrow A\left[x, y, y^{-1}\right] /\left(x-t y^{-1}\right) \rightarrow \mathcal{H}_{Y}^{1}\left(\mathcal{O}_{C}\right) \rightarrow 0
$$

It is convenient to grade these rings with $x$ having degree 1 and $y$ having degree -1 , so that we have the following formula for $\mathcal{H}_{Y}^{1}\left(\mathcal{O}_{C}\right)$ :

$$
\mathcal{H}_{Y}^{1}\left(\mathcal{O}_{C}\right)=A\left[y, y^{-1}\right] / A\left[t y^{-1}, y\right]=\sum_{n=1}^{\infty} y^{-n} A / y^{-n} t^{n} A
$$

The element $x \in \mathcal{O}_{C}$ acts as $t y^{-1}$. We verify that it is not a zero divisor. Since $x$ is homogeneous in our grading, it is sufficient to show that $x$ does not act as a zero divisor on any graded piece of $\mathcal{H}_{Y}^{1}\left(\mathcal{O}_{C}\right)$. Let $y^{-n} c \in y^{-n} A$ represent an arbitrary element of $y^{-n} A / y^{-n} t^{n} A$. If $x c y^{-n}=0$ then $c t=b t^{n+1}$ for some $b \in A$. But $t$ is not a zero divisor in $A$, so this implies $c=b t^{n}$, whence $c y^{-n}$ represents 0 in $\mathcal{H}_{Y}^{1}\left(\mathcal{O}_{C}\right)$. It follows that $x$ is not a zero divisor in $\mathcal{H}_{Y}^{1}\left(\mathcal{O}_{C}\right)$, so $\mathcal{H}_{Z}^{0} \mathcal{H}_{Y}^{1}\left(\mathcal{O}_{C}\right)=0$, as required.

Remark 3.6.2. In the case where $A$ is noetherian, we could observe that $x$ and $y$ form a regular sequence, and therefore their vanishing locus has depth 2 [Gro05, Proposition III.3.3].

Corollary 3.6.3. Let $C$ be a nodal curve over $S$ and let $j: U \rightarrow C$ be the inclusion of the open subset of $C$ where $C$ is smooth over $S$. Assume that every node of $Z$ has a local equation $x y-t$ where $t$ is not a zero divisor in $\mathcal{O}_{C}$. Then $\mathcal{O}_{C} \rightarrow j_{*} \mathcal{O}_{U}$ is an isomorphism. 
Proof. The assertion is local in the étale topology, so we may assume that $S=\operatorname{Spec} A$ and that $C=$ $\operatorname{Spec} A[x, y] /(x y-t)$ where $t$ is not a zero divisor in $A$. Then the corollary is precisely the assertion of the lemma.

Proposition 3.6.4. Let $C$ be a logarithmic curve over the spectrum, $S$, of a valuation ring. Assume that $S$ has the maximal logarithmic structure extending a valuative logarithmic structure on its generic point, $\eta$. Let $j: C_{\eta} \rightarrow C$ be the inclusion of the generic fiber. Then the following maps are isomorphisms:

$$
\begin{gathered}
M_{C} \rightarrow \mathcal{O}_{C} \underset{j_{*} \mathcal{O}_{C_{\eta}}}{\times} M_{C_{\eta}} \\
M_{C}^{\mathrm{gp}} \rightarrow j_{*} M_{C_{\eta}}^{\mathrm{gp}}
\end{gathered}
$$

In other words, the logarithmic structure of $C$ is the maximal extension of that of $C_{\eta}$.

Proof. Our assertion is étale-local on $S$, so we may assume $S$ is the spectrum of a henselian valuation ring.

Throughout the proof, we will write $j: \eta \rightarrow S$ denote the inclusion of the generic point, as well as the inclusion of the generic fiber $C_{\eta} \subset C$. Note that $j_{*}\left(\mathcal{O}_{\eta}^{*}\right) / \mathcal{O}_{S}^{*}$ is the value group of $S$; we denote it $V$.

We write $M_{C}$ for the logarithmic structure of $C$ and $N_{C}$ for the pushforward logarithmic structure $\mathcal{O}_{C} \times_{j_{*} \mathcal{O}_{C_{\eta}}} j_{*} M_{C_{\eta}}$ extending $M_{C_{\eta}}$. By the universal property of $N_{C}$, there is a morphism $M_{C} \rightarrow N_{C}$, which we aim to verify is an isomorphism. It will be sufficient to check that $\bar{M}_{C} \rightarrow \bar{N}_{C}$ is an isomorphism.

We check first that (3.6.4.2) is an isomorphism. We have a commutative diagram with exact rows:

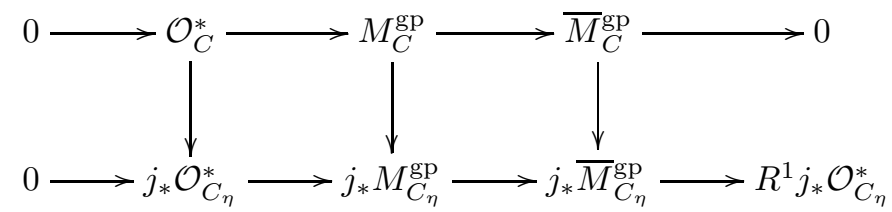

Let $\mathscr{E}$ denote the kernel of $\bar{M}_{C}^{\mathrm{gp}} \rightarrow j_{*} \bar{M}_{C_{\eta}}^{\mathrm{gp}}$ and let $\mathscr{D}$ denote the cokernel of $\mathcal{O}_{C}^{*} \rightarrow j_{*} \mathcal{O}_{C_{\eta}}^{*}$. We will show that (3.6.4.2) is an isomorphism by showing $\mathcal{O}_{C}^{*} \rightarrow j_{*} \mathcal{O}_{C_{\eta}}^{*}$ is injective (this is immediate), $\bar{M}_{C}^{\mathrm{gp}} \rightarrow j_{*} \bar{M}_{C_{\eta}}^{\mathrm{gp}}$ is surjective, and that the connecting homomorphism $\mathscr{E} \rightarrow \mathscr{D}$ is an isomorphism.

We verify that the $\bar{M}_{C}^{\mathrm{gp}} \rightarrow j_{*} \bar{M}_{C_{\eta}}^{\mathrm{gp}}$ is surjective. Indeed, let $\mathfrak{X}_{\xi} \rightarrow \mathfrak{X}_{\eta}$ be the contraction of dual graphs associated to a specialization $\eta \rightsquigarrow \xi$ in $S$. A local section of $\bar{M}_{C}^{\text {gp }}$ corresponds to function on a subgraph $\Gamma$ of $\mathfrak{X}_{\xi}$ that is linear with integer slope along the edges, and takes values in $\bar{M}_{\xi}^{\mathrm{gp}}$. Let $\Gamma^{\prime}$ be the image of $\Gamma$ under the edge contraction $\mathfrak{X}_{\xi} \rightarrow \mathfrak{X}_{\eta}$. A section of $j_{*} \bar{M}_{C_{\eta}}^{\text {gp }}$ corresponds to a function on $\Gamma^{\prime}$ that is linear with integer slope along the edges and takes values in $\bar{M}_{\eta}^{\text {gp }}$. Locally, $\Gamma$ is either an edge or a vertex and it is immediate from the surjectivity of $\bar{M}_{\eta}^{\mathrm{gp}} \rightarrow \bar{M}_{\xi}^{\mathrm{gp}}$ that linear functions on $\Gamma^{\prime}$ valued in $\bar{M}_{\eta}^{\mathrm{gp}}$ lift to linear functions on $\Gamma$ valued in $\bar{M}_{\xi}^{\mathrm{gp}}$.

Now we check that $\mathscr{E} \rightarrow \mathscr{D}$ is an isomorphism. Sections of $\mathscr{E}$ correspond to functions on the dual graph of the special fiber of $C$, valued in the kernel of $M_{S}^{\mathrm{gp}} \rightarrow M_{\eta}^{\mathrm{gp}}$ and linear along the edges with integral slope. By definition of the maximal extension $M_{S}^{\mathrm{gp}}$, the kernel of the restriction $M_{S}^{\mathrm{gp}} \rightarrow M_{\eta}^{\mathrm{gp}}$ is the value group $V$. Thus sections of $\mathscr{E}$ correspond to piecewise linear functions on the dual graph, valued in $V$.

The sections of $\mathscr{D}$ correspond to invertible sheaves $\mathscr{L}$ on $C$ with specified trivialization $\sigma$ along $C_{\eta}$. Equivalently, $\mathscr{D}$ is the sheaf of Cartier divisors on $C$ whose support is disjoint from $C_{\eta}$. We caution that, because $C$ is not necessarily noetherian, the coefficient of a component $D$ of the special fiber, which is obtained by evaluating $\sigma$ at the generic point $\omega$ of $D$, lies in the value group of the valuation ring $\mathcal{O}_{\omega}$. As $C$ is smooth over $S$ at $\omega$, the value group of $\mathcal{O}_{\omega}$ is $V$ for all components of the special fiber. Near a node $Z$ with local equation $x y=t$, the trivialization $\sigma$ must take the form $x^{a} y^{b} \sigma_{0}$, for some integers $a$ and $b$, where $\sigma_{0}$ is a local trivialization of $\mathscr{L}$. Indeed, if $a$ and $b$ are the orders of zero or pole of $\sigma$ along the branches $\{x=0\}$ and $\{y=0\}$ of the node, then $x^{-a} y^{-a} \sigma_{0}^{-1} \sigma$ is a unit of $\mathcal{O}_{C-Z}$, hence extends uniquely to a unit of $\mathcal{O}_{C}$ by Proposition 3.6.3. If this node connects components $\omega$ and $\psi$ then $\operatorname{ord} \sigma(\omega)=\operatorname{ord} \sigma(\psi)+(b-a) \delta$, where $\delta$ is the image of $t$ in the value group $V$. Therefore sections of $\mathscr{D}$ also correspond to functions on the dual graph of the special fiber of $C$ that are valued in $V$ and are linear with integral slope along the edges.

We leave it to the reader to verify that the map $\mathscr{E} \rightarrow \mathscr{D}$ constructed above commutes with the identifications of $\mathscr{E}$ and $\mathscr{D}$ with sheaves of piecewise linear functions and is therefore an isomorphism. This concludes the demonstration that (3.6.4.2) is an isomorphism. 
To prove that (3.6.4.1) is an isomorphism, it is sufficient to show that $\bar{M}_{C} \rightarrow \bar{N}_{C}$ is an isomorphism. Note first that we have

$$
\bar{M}_{C}^{\mathrm{gp}} \rightarrow \bar{N}_{C}^{\mathrm{gp}} \subset j_{*}\left(M_{C}^{\mathrm{gp}}\right) / \mathcal{O}_{C}^{*}
$$

and we have just seen that the composition is an isomorphism. It follows that $\bar{N}_{C}^{g p}=\bar{M}_{C}^{g p}$ and therefore that sections of $\bar{N}_{C}$ correspond to piecewise linear functions on the dual graph of $C$. It is immediate that (3.6.4.1) holds away from the nodes of the special fiber of $C$ over $S$, because there we have $\bar{M}_{C}=\pi^{-1} \bar{M}_{S}$. On the other hand, a local section of $\bar{M}_{C}^{\mathrm{gp}}$ lies in $\bar{M}_{C}$ if and only if its restriction to the complement of the nodes lies in $\bar{M}_{C}$. This can be seen from the interpretation in terms of piecewise linear functions on the dual graph, where a section valued in $\bar{M}_{S}^{\mathrm{gp}}$ is $\geq 0$ if and only if its restriction to each vertex is $\geq 0$. We conclude that $\bar{N}_{C}$ coincides with $\bar{M}_{C}$ away from the nodes of the special fiber of $C$, so they agree everywhere.

\section{The logarithmic Compactification of the Abel-Jacobi SeCtion}

\subsection{Tropical line bundles.}

Definition 4.1.1. For any logarithmic scheme $X$, we define

$$
\begin{aligned}
\mathbf{G}_{m}^{\log }(X) & =\Gamma\left(X, M_{X}^{\mathrm{gp}}\right) \\
\mathbf{G}_{m}^{\text {trop }}(X) & =\Gamma\left(X, \bar{M}_{X}^{\mathrm{gp}}\right)
\end{aligned}
$$

and call these, respectively, the logarithmic multiplicative group and the tropical multiplicative group. We also call $\mathbf{G}_{m}^{\text {trop }}$ the tropical line.

Remark 4.1.2. As described in Section 3.3, maps from a curve $C$ over $S$ into $\mathbf{G}_{m}^{\text {trop }}$ correspond to piecewise linear functions on the tropicalization, valued in $\bar{M}_{S}^{\mathrm{gp}}$. Notably, there is no balancing condition, so we use the term 'tropical' here with some license.

It seems difficult to characterize balanced functions on the tropicalization sheaf theoretically on $C$. The image of $\Gamma\left(C, M_{C}^{\mathrm{gp}}\right) \rightarrow \Gamma\left(C, \bar{M}_{C}^{\mathrm{gp}}\right)$ consists of some, but in general not all, of the balanced functions on $C$; there is no subsheaf of $\bar{M}_{C}^{\mathrm{gp}}$ whose sections correspond to balanced piecewise linear functions on the tropicalization, because balancing is not a local condition in the étale (or Zariski) topology on $C$. In [MW18], balanced functions are characterized as the kernel of the homomorphism $\Gamma\left(C, \bar{M}_{C}^{\mathrm{gp}}\right) \rightarrow \operatorname{NS}(C)$, where $\mathrm{NS}(C)$ is the Néron-Severi group. This definition seems to generalize well (for example, to surfaces), but it is not a local condition in the Zariski topology on $C$.

Neither $\mathbf{G}_{m}^{\log }$ nor $\mathbf{G}_{m}^{\text {trop }}$ is representable by an algebraic stack with a logarithmic structure. However, $\mathbf{G}_{m}^{\log }$ has a logarithmic modification that is representable by $\mathbf{P}^{1}$ with its toric logarithmic structure [RW20, Proposition 1]. Likewise, $\mathbf{G}_{m}^{\text {trop }}$ has a logarithmic modification that is representable by the stack $\left[\mathbf{P}^{1} / \mathbf{G}_{m}\right]$.

Remark 4.1.3. It may seem odd that a logarithmic modification of the functor $\mathbf{G}_{m}^{\text {trop }}$ should be representable by the stack $\left[\mathbf{P}^{1} / \mathbf{G}_{m}\right]$, but this is a typical phenomenon in logarithmic geometry. When $\left[\mathbf{P}^{1} / \mathbf{G}_{m}\right]$ is given the right logarithmic structure, it actually does represent a functor and not merely an algebraic stack, because the logarithmic structure serves to rigidify the moduli problem. Put another way, when $\mathbf{P}^{1}$ is given its toric logarithmic structure, $\mathbf{G}_{m}$ acts on it without fixed points (in a logarithmic sense). Put still another way, the map from $\left[\mathbf{P}^{1} / \mathbf{G}_{m}\right]$ to Olsson's stack of logarithmic structures [Ols03] is representable.

A map $X \rightarrow \mathbf{G}_{m}^{\text {trop }}$ corresponds to a section $\alpha$ of $\bar{M}_{X}^{\text {gp }}$, and therefore to a $\mathbf{G}_{m}$-torsor $\mathcal{O}_{X}^{*}(\alpha)$ on $X$. The identity map $\mathbf{G}_{m}^{\text {trop }} \rightarrow \mathbf{G}_{m}^{\text {trop }}$ should therefore induce a universal $\mathbf{G}_{m}$-torsor on $\mathbf{G}_{m}^{\text {trop }}$. The following lemma characterizes it.

Proposition 4.1.4. The universal $\mathbf{G}_{m}$-torsor over $\mathbf{G}_{m}^{\text {trop }}$ is $\mathbf{G}_{m}^{\log }$.

Proof. Suppose that $X \rightarrow \mathbf{G}_{m}^{\text {trop }}$ corresponds to a section $\alpha$ of $\bar{M}_{X}^{\text {gp }}$. The associated $\mathbf{G}_{m}$-torsor is the sheaf of lifts of $\alpha$ to $M_{X}^{\mathrm{gp}}$. This is precisely the same as the sheaf of lifts of $X \rightarrow \mathbf{G}_{m}^{\text {trop }}$ to $\mathbf{G}_{m}^{\log }$.

Definition 4.1.5. Let $S$ be a logarithmic scheme. A (piecewise linear) tropical line bundle over $S$ is a $\mathbf{G}_{m}^{\text {trop }}$-torsor on $S$ and a logarithmic line bundle is a $\mathbf{G}_{m}^{\log }$-torsor over $S .{ }^{3}$ We will drop the words "piecewise linear" in this paper, since we will not encounter any other sort of tropical line bundle here.

\footnotetext{
${ }^{3}$ In [MW18], the definition of a logarithmic line bundle includes an additional bounded monodromy condition that does not concern us here.
} 
In other words, a tropical line bundle is a torsor under $\bar{M}_{S}^{\mathrm{gp}}$. We note that the sections of a tropical line bundle $\mathcal{P}$ over $S$ are equipped with a partial order, in which $\beta \leq \gamma$ if there is some $\delta \in \Gamma\left(S, \bar{M}_{S}\right)$ such that $\beta+\delta=\gamma$.

If $P$ is a logarithmic line bundle over $S$ then $P$ carries a natural $\mathbf{G}_{m}$-action and $P / \mathbf{G}_{m}$ is a tropical line bundle over $S$. This is the geometric manifestation of the morphism on first cohomology:

$$
H^{1}\left(S, M_{S}^{\mathrm{gp}}\right) \rightarrow H^{1}\left(S, \bar{M}_{S}^{\mathrm{gp}}\right)
$$

\subsection{Logarithmic divisors on logarithmic curves.}

Definition 4.2.1. Let $C$ be a logarithmic curve over a logarithmic scheme $S$. A logarithmic divisor on $C$ is a tropical line bundle $\mathcal{P}$ over $S$ and an $S$-morphism $C \rightarrow \mathcal{P}$. We write Div for the stack in the strict étale topology on logarithmic schemes whose $S$-points are triples $(C, \mathcal{P}, \alpha)$ where $C$ is a logarithmic curve over $S$, where $\mathcal{P}$ is a tropical line bundle over $S$, and where $\alpha: C \rightarrow \mathcal{P}$ is an $S$-morphism.

Example 4.2.2. Let $S$ be a logarithmic scheme whose underlying scheme is the spectrum of an algebraically closed field and let $C$ be a logarithmic curve over $S$. Then $\operatorname{Div}(C / S)$ is the set of piecewise linear functions on the tropicalization of $C$ that are linear with integer slope along the edges and take values in $\bar{M}_{S}^{\mathrm{gp}}$ (see Section 3.3.3), up to translation by constants.

The following proposition gives a more concrete formulation of Example 4.2.2:

Proposition 4.2.3. Let $\pi: C \rightarrow S$ be a family of logarithmic curves. Then $\operatorname{Div}(C / S)=\Gamma\left(S, \pi_{*}\left(\bar{M}_{C}^{\mathrm{gp}}\right) / \bar{M}_{S}^{\mathrm{gp}}\right)$.

Proof. Let $\mathcal{P}$ be a tropical line over $S$. Locally in $S$ we may choose an isomorphism between $\mathcal{P}$ and $\mathbf{G}_{m}^{\text {trop }}$, which is unique up to addition of a section of $\bar{M}_{S}^{\mathrm{gp}}$. Then a map $C \rightarrow \mathcal{P} \simeq \mathbf{G}_{m}^{\text {trop }}$ is identified with a global section of $\bar{M}_{C}^{\mathrm{gp}}$, up to translation by $\bar{M}_{S}^{\mathrm{gp}}$.

It is possible to separate Div into components using several locally constant data. The first is the genus of the curve in question. Second, the slopes of the piecewise linear function introduced in Example 4.2.2 on the legs of the tropicalization are locally constant in families. If $\mathbf{a}=\left(a_{1}, \ldots, a_{n}\right)$ is a vector of integers, we write $\mathbf{D i v}_{g, \mathbf{a}}$ for the component of the $S_{n}$ cover of Div where the curve has genus $g$, the marked points are labeled by the integers $1, \ldots, n$, and the outgoing slope of the piecewise linear function on the tropicalization on the $i$ th leg is $a_{i}$.

Theorem 4.2.4. Div is representable by an algebraic stack with a logarithmic structure that is locally of finite presentation.

Proof. We employ the standard approach using Gillam's criteria: first we consider the stack $\log \left(\operatorname{Div}_{g, \mathbf{a}}\right)$ over schemes, whose objects are the same as those of $\mathbf{D i v}_{g, \mathbf{a}}$ but whose arrows are only the cartesian arrows over schemes. Equivalently, if $S$ is a scheme, then an $S$-point of $\log \left(\operatorname{Div}_{g, \mathbf{a}}\right)$ is the choice of a $\operatorname{logarithmic}$ structure $M_{S}$ on $S$ and an $\left(S, M_{S}\right)$-point of $\operatorname{Div}_{g, \mathbf{a}}$. Once we have $\operatorname{shown} \log \left(\mathbf{D i v}_{g, \mathbf{a}}\right)$ is an algebraic stack, we equip it with the minimal logarithmic structure and identify $\mathbf{D i v}_{g, \mathbf{a}}$ as an open substack.

We begin by showing $\log \left(\mathbf{D i v}_{g, \mathbf{a}}\right)$ is algebraic. Denote by $\mathfrak{M}_{g, n}^{\log }$ the moduli stack of logarithmic curves of genus $g$ with $n$ marked points. Working relative to $\log \left(\mathfrak{M}_{g, n}^{\log }\right)$, we may assume a fixed logarithmic curve $\pi: C \rightarrow S$ has been given. As the formation of $\pi_{*} \bar{M}_{C}^{\mathrm{gp}}$ in the small étale topology commutes with base change, so does the formation of $\pi_{*}\left(\bar{M}_{C}^{\mathrm{gp}}\right) / \bar{M}_{S}^{\mathrm{gp}}$. That is, if $f: T \rightarrow S$ is a strict morphism of logarithmic schemes, then $\pi_{*}\left(\bar{M}_{C_{T}}^{\mathrm{gp}}\right) / \bar{M}_{T}^{\mathrm{gp}}=f^{*}\left(\pi_{*}\left(\bar{M}_{C}^{\mathrm{gp}}\right) / \bar{M}_{S}^{\mathrm{gp}}\right)$. Therefore $\mathbf{L o g}\left(\mathbf{D i v}_{g, \mathbf{a}}\right)$ is the pullback to the large étale site of the sheaf $\pi_{*}\left(\bar{M}_{C}^{\mathrm{gp}}\right) / \bar{M}_{S}^{\mathrm{gp}}$ on the small étale site. It is therefore representable by the espace étalé [Mil80, Theorem V.1.5] of $\pi_{*}\left(\bar{M}_{C}^{\mathrm{gp}}\right) / \bar{M}_{S}^{\mathrm{gp}}$ over $\log \left(\mathfrak{M}_{g, n}^{\mathrm{log}}\right)$. This must be locally of finite presentation, since it is étale over $\log \left(\mathfrak{M}_{g, n}^{\log }\right)$.

Recall that a tuple $\left(S^{\prime}, C^{\prime}, \alpha^{\prime}\right)$, where $S^{\prime}$ is a logarithmic scheme, $C^{\prime}$ is a logarithmic curve over $S^{\prime}$, and $\alpha^{\prime}$ is a logarithmic divisor on $C^{\prime}$, is called minimal if, whenever $(S, C, \alpha)$ is pulled back from $\left(S^{\prime}, C^{\prime}, \alpha^{\prime}\right)$ by a morphism $S \rightarrow S^{\prime}$ that is the identity on underlying schemes, and $(S, C, \alpha) \rightarrow\left(S^{\prime \prime}, C^{\prime \prime}, \alpha^{\prime \prime}\right)$ is a second morphism that is the identity on underlying schemes, there is a unique morphism $\left(S^{\prime \prime}, C^{\prime \prime}, \alpha^{\prime \prime}\right) \rightarrow\left(S^{\prime}, C^{\prime}, \alpha^{\prime}\right)$ 
completing the triangle below (see [Gil12] and [Wis16, Appendix B] for more details):

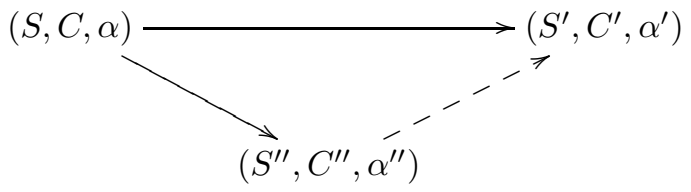

By [Wis16, Proposition B.1], Div $\mathbf{~}_{g, \mathbf{a}}$ is induced from a logarithmic structure on a moduli problem on logarithmic schemes if and only if every object of $\mathbf{D i v}_{g, \mathbf{a}}$ admits a morphism to a minimal object that is the identity on underlying schemes, and that the pullback of a minimal object under an arbitrary morphism of schemes is still minimal. In the language of op. cit., $G=\mathbf{D i v}_{g, \mathbf{a}}$ is representable by the substack of minimal objects $F \subset \log \left(\mathbf{D i v}_{g, \mathbf{a}}\right)$. Furthermore, [Wis16, Theorem B.2] implies that, if the minimal objects of $\mathbf{D i v}_{g, \mathbf{a}}$ have coherent logarithmic structures then the substack of minimal objects, $F \subset \log \left(\mathbf{D i v}_{g, \mathbf{a}}\right)$, is open. We may therefore conclude that $\mathbf{D i v}_{g, \mathbf{a}}$ is representable by an algebraic stack with a logarithmic structure once we show that (i) all objects of $\mathbf{D i v}_{g, \mathbf{a}}$ admit morphisms to minimal objects, (ii) minimal objects are preserved by schematic base change, and (iii) minimal logarithmic structures are coherent.

Suppose $\alpha$ is a logarithmic divisor on a logarithmic curve $C$ over a logarithmic scheme $S$, and denote by $N_{S}$ the minimal logarithmic structure on $S$ for the family of curves $C$ (ignoring the logarithmic divisor). The is the same as the logarithmic structure pulled back from the moduli space of curves; the stalk of $\bar{N}_{S}$ at a geometric point $s$ is freely generated by the smoothing parameters of the nodes in the fiber $C_{s}$. We will show that $\alpha$ is actually defined over the image of $N_{S}$ in $M_{S}$. We work in a small neighborhood of a geometric point $s$ of $S$ so that we may view $\alpha$ as a piecewise linear function on the tropicalization of $C_{s}$, well-defined up addition of a constant. Adding a suitable constant to $\alpha$, we can therefore assume that $\alpha$ takes the value 0 at some vertex of the dual graph of $C_{s}$. Since $\alpha$ is linear with integer slopes along the edges, and the lengths of all edges come from the image of $\bar{N}_{S}$, it follows that all values of $\alpha$ lie in the image of $\bar{N}_{S}^{\text {gp }}$, and therefore that $\alpha$ is defined over the image of $\bar{N}_{S}$ in $\bar{M}_{S}$.

Now, let $K \subset \bar{N}_{S}^{\text {gp }}$ be the smallest subgroup such that $\alpha$ can be represented by an element of $\bar{N}_{S}^{\text {gp }} / K$. The subgroup $K$ is generated pointwise as follows: for every directed edge $e$ from a vertex $v$ to a vertex $w$ in the dual graph of $C, \alpha$ determines an element $\delta_{e}=\alpha(w)-\alpha(v) \in \bar{N}_{S}^{\text {gp }}$; the elements of $K$ are the sums $\sum_{e \in \gamma} \delta_{e}$ for each directed loop $\gamma$ in the dual graph. We will see that the image of $\bar{N}_{S}$ in $\bar{N}_{S}^{\mathrm{gp}} / K$ is the minimal monoid.

If we write $\left(S^{\prime}, C^{\prime}, \alpha^{\prime}\right)$ for the object of $\mathbf{D i v}_{g, \mathbf{a}}$ whose underlying scheme is the same as that of $S$, whose characteristic monoid $\bar{M}_{S^{\prime}}$ is the image of $\bar{N}_{S}$ in $\bar{N}_{S}^{g p} / K$, and where $\alpha^{\prime}$ is the canonical lift of $\alpha$ to $\operatorname{Div}_{g, \mathbf{a}}\left(C^{\prime} / S^{\prime}\right)$ constructed above, then we have a morphism $(S, C, \alpha) \rightarrow\left(S^{\prime}, C^{\prime}, \alpha^{\prime}\right)$. To see that $\left(C^{\prime}, S^{\prime}, \alpha^{\prime}\right)$ is minimal, consider a diagram (4.2.4.1). Since $N_{S}$ is minimal for the family of logarithmic curves, we have a map $N_{S} \rightarrow M_{S^{\prime \prime}}$ and, by the same reasoning as above, $\alpha^{\prime \prime}$ is defined over the image logarithmic structure. All of the relations in $K$ must hold in $\bar{M}_{S^{\prime \prime}}$ for the same reasons they held in $\bar{M}_{S}$, and therefore the map $\bar{N}_{S}^{\mathrm{gp}} / K \rightarrow \bar{N}_{S}^{\mathrm{gp}}$ factors through $\bar{M}_{S^{\prime \prime}}^{\mathrm{gp}}$. This completes the diagram (4.2.4.1) and shows that $\left(S^{\prime}, C^{\prime}, \alpha^{\prime}\right)$ is minimal.

This construction is compatible with base change, so defines the minimal structures for our logarithmic moduli problem and therefore an algebraic stack. Therefore $\mathbf{D i v}_{g, \mathbf{a}}$ can be described by a logarithmic structure on a schematic moduli problem. Since the construction produces coherent logarithmic structures (we have given explicit finitely generated charts), $\mathbf{D i v} \mathbf{v}_{g, \mathbf{a}}$ is in fact the logarithmic moduli problem associated to an open substack of $\log \left(\operatorname{Div}_{g, \mathbf{a}}\right)$. This substack is locally of finite presentation because $\log \left(\operatorname{Div} \mathbf{v}_{g, \mathbf{a}}\right)$ is.

Proposition 4.2.5. The forgetful morphism $\mathbf{D i v}_{g, \mathbf{a}} \rightarrow \mathfrak{M}_{g, n}^{\log }$ to the moduli space of logarithmic curves is logarithmically étale.

Proof. This is immediate from the fact that $\pi_{*}\left(\bar{M}_{C}^{\mathrm{gp}}\right) / \bar{M}_{S}^{\mathrm{gp}}$ is an étale sheaf.

Corollary 4.2.6. $\operatorname{Div}_{g, \mathbf{a}}$ is logarithmically smooth and birational to $\mathfrak{M}_{g, n}^{\log }$. 
Proof. As $\mathbf{D i v}_{g, \mathbf{a}}$ is logarithmically étale over $\mathfrak{M}_{g, n}^{\log }$, and $\mathfrak{M}_{g, n}^{\log }$ is logarithmically smooth (over $\mathbf{Z}$ with the trivial logarithmic structure), so is $\mathbf{D i v}_{g, \mathbf{a}}$. It is an isomorphism over the locus where the logarithmic structures are trivial, and in a logarithmically smooth logarithmic scheme, the open subset where the logarithmic structure is trivial is dense.

\subsection{The Abel-Jacobi section.}

Definition 4.3.1. We write $\mathbf{P i c}_{g, n}$ for the stack on logarithmic schemes whose $S$-points are pairs $(C, L)$, where $\pi: C \rightarrow S$ is a logarithmic curve over $S$ and $L$ is a section over $S$ of $\pi_{*}\left(\mathrm{BG}_{m C}\right) / \mathrm{BG}_{m S}=R^{1} \pi_{*} \mathcal{O}_{C}^{*}$.

In the absence of logarithmic structures, it is well-known that $\mathbf{P i c} \mathbf{i}_{g, n}$ is representable by an algebraic stack, provisionally denoted $P$, and that the projection to $\mathfrak{M}_{g, n}$ is representable by algebraic spaces. It can be seen easily that the morphism of functors $\mathbf{P i c} \mathbf{c}_{g, n} \rightarrow \mathfrak{M}_{g, n}$ is strict, and therefore that if $P$ is equipped with the logarithmic structure pulled back from $\mathfrak{M}_{g, n}$ then $P$ represents $\mathbf{P i c} \mathbf{c}_{g, n}$.

We construct a canonical map aj: $\operatorname{Div}_{g, \mathbf{a}} \rightarrow \mathbf{P i c}_{g, n}$. Given an object $(\pi: C \rightarrow S, \alpha)$ of Div over a logarithmic scheme $S$, pushing forward the exact sequence $0 \rightarrow \mathcal{O}_{C}^{*} \rightarrow M_{C}^{\mathrm{gp}} \rightarrow \bar{M}_{C}^{\mathrm{gp}} \rightarrow 0$ along $\pi$ results in a commutative diagram with exact arrows:

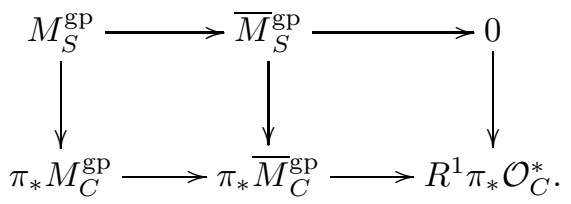

Upon taking quotients in the vertical direction, this yields an exact sequence:

$$
\pi_{*}\left(M_{C}^{\mathrm{gp}}\right) / M_{S}^{\mathrm{gp}} \rightarrow \pi_{*}\left(\bar{M}_{C}^{\mathrm{gp}}\right) / \bar{M}_{S}^{\mathrm{gp}} \rightarrow R^{1} \pi_{*} \mathcal{O}_{C}^{*} .
$$

The second arrow in this exact sequence produces a line bundle class aj $(\alpha)$ in $\operatorname{Pic}(C)$, inducing our desired morphism.

Theorem 4.3.2. The morphism aj: $\mathbf{D i v} \mathbf{v}_{g, \mathbf{a}} \rightarrow \mathbf{P i c}_{g, n}$ is finite, unramified on the underlying algebraic stacks, and logarithmically a monomorphism.

Remark 4.3.3. The logarithmic structure of $\mathbf{D i v} g, \mathbf{a}$ is not necessarily saturated, so aj will be finite but not necessarily an embedding if we work with saturated logarithmic structures.

Proof. Since both source and target are locally of finite presentation, we may demonstrate that aj is finite by showing it

(1) is quasicompact,

(2) is locally quasifinite, and

(3) satisfies the valuative criterion for properness.

Local quasifiniteness follows from being unramified, so the the theorem will follow from the following four lemmas.

Lemma 4.3.3.1. The map aj : $\mathbf{D i v}_{g, \mathbf{a}} \rightarrow \mathbf{P i c}_{g, n}$ is a logarithmic monomorphism.

Proof. We learned the following argument from Lionel Levine. This is a local assertion, so we may assume that we have a logarithmic curve $C$ over $S$ and a geometric point $s$ of $S$ such that an element of $\operatorname{Div}_{g, \mathbf{a}}(C)$ corresponds to a piecewise linear function on the tropicalization of $C_{s}$, up to the addition of a constant. Suppose we have two such piecewise linear functions, $\alpha$ and $\beta$, such that $\operatorname{aj}(\alpha)=\operatorname{aj}(\beta)$. Then $\operatorname{aj}(\alpha-\beta)$ is pulled back from the base. We argue that this implies $\alpha-\beta$ is a constant function.

Indeed, because aj $(\alpha-\beta)$ is pulled back from the base, it has degree zero on every component of $C$. The degree of aj $(\alpha-\beta)$ on a component is the sum of the outgoing slopes of $\alpha-\beta$ from the corresponding vertex. Therefore the sum of the outgoing slopes of $\alpha-\beta$ is zero at every vertex, so $\alpha-\beta$ is a harmonic function. On the other hand, it is bounded, because $\alpha$ and $\beta$ have the same outgoing slopes a on the legs of the dual graph. Therefore $\alpha-\beta$ is a bounded, harmonic function on the tropicalization, hence is constant.

Lemma 4.3.3.2. The map aj: $\mathbf{D i v}_{g, \mathbf{a}} \rightarrow \mathbf{P i c}_{g, n}$ is unramified on the underlying algebraic stacks (ignoring logarithmic structures). 
Lemma 4.3.3.3. Let $S$ be the spectrum of an algebraically closed field, let $C$ be a logarithmic curve over $S$, and let $L$ be an invertible sheaf over $C$. The fiber of $\mathbf{D i v}_{g, \mathbf{a}} \rightarrow \mathbf{P i c}_{g, n}$ over $(S, C, L)$ is a discrete set of points. It is in bijection with the set of assignments of a slope $\lambda_{q} \in \mathbf{Z}$ to each edge $e$ of the dual graph of $C$ satisfying the following condition: let $D$ be the normalization of all nodes of $C$ where $\lambda_{q} \neq 0$; then $\left.L\right|_{D} \simeq \mathcal{O}_{D}\left(\sum \lambda_{q} q\right)$, with the sum taken over all edges $q$ of the dual graph of $D$ created by normalization, identified with special points of $D$ and oriented outwards. In particular, aj: $\mathbf{D i v} \mathbf{v}_{g, \mathbf{a}} \rightarrow \mathbf{P i c}_{g, n}$ is unramified.

Proof. We thank Sebastian Bozlee for his help with this argument, and David Holmes for alerting us to a missing condition in the statement.

By definition, a $T$-point of the fiber of aj over $(S, C, L)$ is a morphism of schemes $f: T \rightarrow S$, a logarithmic structure $M_{T}$ on $T$, making $C_{T}$ into a logarithmic curve over $T$, and a piecewise linear function $\alpha$ on the dual graph of $C$ taking values in $\bar{M}_{T}^{\mathrm{gp}}$, and taken up to the addition of constants, such that $\left.\mathcal{O}_{C_{T}}(\alpha) \simeq L\right|_{C_{T}}$.

The slopes $\lambda_{q} \in \mathbf{Z}$ of $\alpha$ along the edges of the dual graph of $C$ are locally constant on the fiber. Isolating one connected component, we may treat them as fixed. We note that these slopes determine the characteristic monoid $\bar{M}_{T}$ of the logarithmic structure $M_{T}$, but not the logarithmic structure itself.

We will refer to a union of components $D$, as in the statement of the lemma, as a level component of $C$. Certainly the component of the fiber of aj with slopes $\lambda_{q}$ will be empty if there is a level component $D$ of $C$ such that $\left.L\right|_{D}$ is not isomorphic to $\mathcal{O}_{D}\left(\sum \lambda_{q} x_{q}\right)$, where the sum is taken over edges exiting the vertex of the dual graph corresponding to $D$. Therefore we may also assume that $\left.\mathcal{O}_{D}\left(\sum \lambda_{q} x_{q}\right) \simeq L\right|_{D}$ for every level component $D$.

We will now argue that, with $\lambda_{q} \in \mathbf{Z}$ fixed as above such that $\mathcal{O}_{D}\left(\sum \lambda_{q} q\right)$ for each level component $D$ of $C$, there is a unique choice of minimal logarithmic structure $M_{T}$ with characteristic monoid $\bar{M}_{T}$ on $C_{T}$ such that $\mathcal{O}_{C_{T}}(\alpha) \simeq L$. It will then follow that the component of $\mathrm{aj}^{-1}(S, C, L)$ with these fixed slopes $\lambda_{q}$ is a single reduced point.

Let $\Gamma$ be the quotient of the dual graph of $C$ in which the vertices correspond to the level components of $C$ and let $\tilde{\Gamma}$ be its universal cover. Let $\rho: \tilde{C} \rightarrow C$ be the étale cover of $C$ corresponding to the universal cover $\tilde{\Gamma} \rightarrow \Gamma$. The $\lambda_{q}$, when pulled back to $\tilde{\Gamma}$, determine a piecewise linear function $\tilde{\alpha}$ on $\tilde{\Gamma}$ taking values in $\bar{M}_{S}^{\mathrm{gp}}$ and having integral slopes along the edges. This $\tilde{\alpha}$ is unique up to the addition of a constant from $\bar{M}_{S}^{\mathrm{gp}}$.

The isomorphisms $\left.L\right|_{D} \simeq \mathcal{O}_{D}\left(\sum_{q \in D} \lambda_{q} q\right)$ imply that $\rho^{*} L \simeq \mathcal{O}_{\tilde{C}}(\tilde{\alpha})$. Since $\tilde{C}$ is a connected and satisfies the valuative criterion for properness, this isomorphism is unique up to a global scalar. Since $\rho^{*} L$ descends to $C$, we have identifications $\gamma^{*} \rho^{*} L=\rho^{*} L$ for all $\gamma \in \pi_{1}(\Gamma)$. On the other hand, $\gamma^{*} \mathcal{O}_{\tilde{C}}(\tilde{\alpha})=\mathcal{O}_{\tilde{C}}\left(\gamma^{-1} \tilde{\alpha}\right)$ where $\gamma^{-1} \tilde{\alpha}$ is the piecewise linear function given by the following formula for each level component $\tilde{D}$ of $\tilde{C}$ :

$$
\gamma^{-1} \tilde{\alpha}(\tilde{D})=\tilde{\alpha}(\gamma \cdot \tilde{D})=\tilde{\alpha}(\tilde{D})+\sum_{q \in \gamma} \lambda_{q} \delta_{q}
$$

The sum is taken over the oriented edges of $\gamma$ and $\delta_{q} \in \bar{M}_{S}$ is the length of the edge $q$. Thus the isomorphisms $\gamma^{*} \mathcal{O}_{\tilde{C}}(\tilde{\alpha}) \simeq \mathcal{O}_{\tilde{C}}(\tilde{\alpha})$ are specified uniquely by trivializations of the invertible sheaves

$$
\mathcal{O}_{S}\left(\sum_{q \in \gamma} \lambda_{q} \delta_{q}\right)
$$

If $\gamma$ represents a nontrivial element of $H_{1}(\Gamma)$ then the $\lambda_{q}$ are all nonzero for $q \in \gamma$, by the construction of $\Gamma$. It follows (for example, by the choice of a spanning tree) that the homomorphism

$$
H_{1}(\Gamma) \rightarrow \bar{M}_{S}^{\mathrm{gp}}: \gamma \mapsto \sum_{q \in \gamma} \lambda_{q} \delta_{q}
$$

is injective. Therefore these trivializations are precisely the data necessary to build a quotient logarithmic structure of $M_{S}$ in which $\sum_{q \in \gamma} \lambda_{q} \delta_{q}=0$ for all $\gamma \in \pi_{1}(\Gamma)$. The equations $\sum_{q \in \gamma} \lambda_{q} \delta_{q}=0$ are exactly those required to produce a minimal logarithmic structure on $S$ such that $\tilde{\alpha}$ descends to a piecewise linear function on $\Gamma$ valued in its characteristic monoid.

Lemma 4.3.3.4. The map aj: $\mathbf{D i v}_{g, \mathbf{a}} \rightarrow \mathbf{P i c}_{g, n}$ satisfies the valuative criterion for properness.

Proof. Suppose that $C \rightarrow S$ is a family of logarithmic curves, where $S$ is the spectrum of a valuation ring, $j: \eta \rightarrow S$ is the inclusion of its generic point, and the logarithmic structure of $S$ is the maximal extension of 
the logarithmic structure on $\eta$ (as in Definition 3.5.1). That is, $M_{S}=j_{*} M_{\eta} \times_{j_{*} \mathcal{O}_{\eta}} \mathcal{O}_{S}$. We consider a lifting problem:

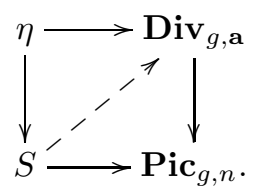

Making a ramified base change, we can assume that $\bar{M}_{\eta}$ is a constant sheaf on $\eta$.

Let $C_{\eta}$ be the restriction of $C$ to $\eta$, and let $C_{0}$ be the special fiber. The diagram gives a line bundle $L$ on $C$ and a section $\alpha_{\eta} \in \Gamma\left(C_{\eta}, \bar{M}_{C_{\eta}}\right)$ such that $\operatorname{aj}\left(\alpha_{\eta}\right)=\left.L\right|_{\eta}$.

There is a commutative diagram with exact rows:

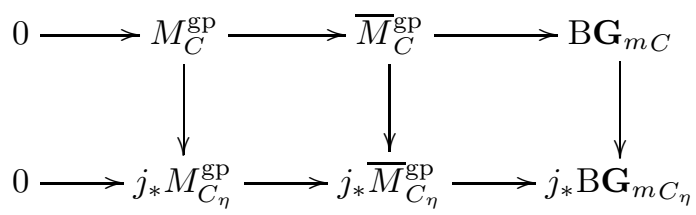

By Proposition 3.6.4, the map $M_{C}^{\mathrm{gp}} \rightarrow j_{*} M_{C_{\eta}}^{\mathrm{gp}}$ is an isomorphism, so the square on the right is cartesian. The line bundle $L$ is a section of $\mathbf{B G}_{m C}$, the section $\alpha_{\eta}$ lies in $j_{*} \bar{M}_{C_{\eta}}^{\mathrm{gp}}$, and these have the same image in $j_{*} \mathrm{BG}_{m C_{\eta}}$, by assumption. Therefore there is a unique section $\alpha$ of $\bar{M}_{C}^{\mathrm{gp}}$ inducing both $\alpha_{\eta}$ and $L$.

This does not yet complete the proof, because $\alpha_{\eta}$ was only well-defined up to addition of a section of $\bar{M}_{\eta}^{\mathrm{gp}}$. Likewise, $L$ was only well-defined up to tensor product by a section of $j_{*} \mathbf{B} \mathbf{G}_{m \eta}$. We must show that $\alpha$ does not depend on these choices. On the other hand, we are only interested in $\alpha$ up to addition of a section of $\bar{M}_{S}^{\mathrm{gp}}$. We once again have a commutative diagram with exact rows (4.3.3.2):

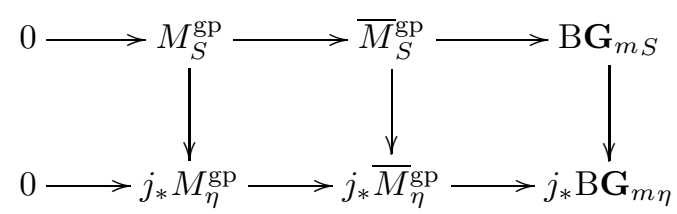

The map $M_{S}^{\mathrm{gp}} \rightarrow j_{*} M_{\eta}^{\mathrm{gp}}$ is again an isomorphism (by definition this time, not by Proposition 3.6.4) so the square on the right is once again cartesian.

This shows that the ambiguity in the $\alpha$ we have produced is precisely the common ambiguity in the original choices of $L$ and $\alpha_{\eta}$, and completes the proof.

Lemma 4.3.3.5. The map aj : $\mathbf{D i v}_{g, \mathbf{a}} \rightarrow \mathbf{P i c}_{g, n}$ is quasicompact.

Proof. We must show that, given a quasicompact base $S$, a family of logarithmic curves $C$ over $S$, and a line bundle $L$ on $C$, the fiber product $S \times \mathbf{P i c}_{g, n} \mathbf{D i v}_{g, \mathbf{a}}$ is quasicompact. We may freely pass to a constructible and étale covers of $S$ and thereby assume that the logarithmic structure of $S$ constant. We can also assume that $C$ has constant dual graph $G$. The multidegree of $L$ is then fixed.

Bounding THE SLOPES. Following [FP18], we argue that there are at most finitely many possibilities for the slopes of a piecewise linear function $\alpha$ on the tropicalization of $C$ such that the multidegree of $\mathcal{O}_{C}(\alpha)$ agrees with the multidegree of $L$.

The degrees of $L$ on each of the irreducible components of $C$ are a tropical divisor - an integer valued function on the vertices of $G$. If $\alpha$ is a piecewise linear function on the tropicalization of $C$ then the degree of aj $(\alpha)$ on a component of $C$ is the deviation from linearity (i.e., the sum of the outgoing slopes) of $\alpha$ at the corresponding vertex of the tropicalization. In effect, we wish to show that there are finitely many piecewise linear functions $\alpha$ with the same associated tropical divisor.

Any choice of $\alpha$ endows the tropicalization of $C$ with an orientation of its edges (the direction of increase of $\alpha$, treating vertices as equivalent when the slope along an edge connecting them is zero), and since there are finitely many ways to orient a finite graph, we may assume one orientation has been fixed. This graph can have no directed loops, for if we had a sequence of vertices $v_{0}, v_{1}, \ldots, v_{n}=v_{0}$ in a directed loop then we 
would have $\alpha\left(v_{i}\right)-\alpha\left(v_{i-1}\right) \in \bar{M}_{S}$ for all $i=1, \ldots, n$. But then $\sum_{i=1}^{n}\left(\alpha\left(v_{i}\right)-\alpha\left(v_{i-1}\right)=0\right.$ implies that all $\alpha\left(v_{i}\right)$ are equal since $\bar{M}_{S}$ is a sharp monoid.

Now we proceed by induction: since the graph has no directed loops, there is at least one vertex with only outwardly directed edges. The degree of $L$ on this component constrains the possible outward slopes of $\alpha$ on the adjacent edges to a finite collection of possibilities. We therefore assume one is fixed. Now delete that vertex and the adjacent edges, adjusting the degree of $L$ on the adjacent components to account for the edges that have been removed. By induction, there are only finitely many possibilities for the slopes.

Bounding THE LOGARITHMiC STRUCture. We may now assume that the slopes of $\alpha$ on the edges of $G$ are fixed. We must still show that the space of lifts of $S \rightarrow \mathbf{P i c}_{g, n}$ to $\mathbf{D i v} \mathbf{v}_{g, \mathbf{a}}$ with these slopes is quasicompact. Any such map will give a quotient $M_{S}^{\prime}$ of the logarithmic structure of $M_{S}$, and the characteristic monoid $\bar{M}_{S}^{\prime}$ of this quotient is determined by the fixed slopes of $\alpha$. Once its characteristic monoid has been determined, the choices of this logarithmic structure vary in a quasicompact family, so we may assume it has been fixed.

Conclusion. Now we have fixed slopes along the edges of $G$ and a logarithmic structure such that these slopes arise from a piecewise linear function. This gives a uniquely determined logarithmic divisor $\alpha$ on $C$, and we wish to show that the inclusion in $S$ of the locus where $\mathcal{O}_{C}(\alpha)$ agrees with $L$ (up to tensor product by a line bundle from the base) is quasicompact. In fact, it is closed, by the Seesaw Theorem [Mum08, Section 5, Corollary 6, p. 54].

The lemmas together complete the proof of Theorem 4.3.2.

We introduce the notation $\operatorname{Div}_{g, \mathbf{a}}^{0}$ for the open substack of $\mathbf{D i v}_{g, \mathbf{a}}$ corresponding to $(C, \alpha)$ such that, when $\alpha$ is regarded as an equivalence class of piecewise linear functions on the tropicalization of $C$, it is actually linear. That is, the outgoing slopes of $\alpha$ at each vertex sum to zero. We also introduce $\mathbf{D i v}_{g, \mathbf{a}}^{\mathrm{c}}$ for the open substack of $\mathbf{D i v}_{g, \mathbf{a}}$ where the sum of the outgoing slopes at a vertex $v$ of the tropicalization of a curve is $2 h-2+m$, where $h$ is the valence of $v$ and $m$ is the number of half-edges incident to $v$.

Proposition 4.3.4. The loci of $(C, \alpha)$ in $\mathbf{D i v}_{g, \mathbf{a}}^{0}$ and in $\mathbf{D i v}_{g, \mathbf{a}}^{\mathrm{can}}$ such that $C$ is of compact type are equivalent to ${ }^{\mathrm{ct}} \mathbf{D i v}_{g, \mathbf{a}}^{0}$ and to ${ }^{\mathrm{ct}} \mathbf{D i v}_{g, \mathbf{a}}^{\mathrm{can}}$, respectively.

Proof. On the compact type locus the dual graph of any fiber of $\pi: C \rightarrow S$ is a tree, making the subgroup $K$ of the minimal logarithmic structure in the proof of Theorem 4.2.4 trivial. Thus the minimal logarithmic structures of $\mathbf{D i v}_{g, \mathbf{a}}$ over the compact type locus are the same as those of ${ }^{c t} \mathfrak{M}_{g, n}^{\log }$. Once the slopes of $\alpha$ on the legs of a tree are specified, there is a unique choice of $\alpha$ that is linear at the vertices (see the proof of Lemma 4.3.3.1) and has the specified slopes on the legs. Therefore, the only extra datum provided by the section $\alpha$ of $\pi_{*}\left(\bar{M}_{C}^{\mathrm{gp}}\right) / \bar{M}_{S}^{\mathrm{gp}}$ is the choice of an integer weight $a_{i}$ at each marked point.

The proof for canonical degrees is similar.

4.4. An example of resolved indeterminacy. We reprise the example of Section 2.2. Let $S_{0}$ be the spectrum of an algebraically closed field, and $C_{0}$ the curve shown in the special fiber of Figure 1 . Let $C$ be a versal deformation of the nodes of $C_{0}$, with base $S$. At least after localizing around $S_{0}$, we can assume that $S$ has two distinct divisors $\Delta_{q}$ and $\Delta_{r}$ where the nodes $q$ and $r$, respectively, persist.

Over the complement of $\Delta_{q} \cap \Delta_{r}$, the line bundle $\mathcal{O}_{C}\left(n p-n p^{\prime}\right)$ has degree zero on the fibers of $C$ and therefore defines a section of $\mathbf{P i c}{ }^{0}(C)$ over $S$. Let $Z$ be the closure of this section in $\mathbf{P i c}^{0}(C)$.

The fiber of $\mathbf{P i c}{ }^{0}(C)$ over $S_{0}$ is a $\mathbf{G}_{m}$-torsor over $\mathbf{P i c}^{0}(D) \times \mathbf{P i c}{ }^{0}(E)$, the projection being pullback to the normalization. The calculations in Section 2.2 show that, if $n \neq 0$, the fiber of $Z_{0}$ over a point of $\mathbf{P i c}^{0}(D) \times \mathbf{P i c}{ }^{0}(E)$ is either empty or is the entire fiber of $\mathbf{P i c}{ }^{0}(C)$ over the same point.

Lemma 4.4.1. If $n \neq 0$, the fiber of $Z_{0}$ over $\left(L_{D}, L_{E}\right)$ is nonempty if and only if $L_{D} \simeq \mathcal{O}_{D}(n p-a q-b r)$ and $L_{E} \simeq \mathcal{O}_{E}\left(-n p^{\prime}+a q+b r\right)$ where $a$ and $b$ are integers of the same sign such that $a+b=n$.

Proof. There is a symmetry between $n<0$ and $n>0$, so we assume without loss of generality that $n>0$.

If the fiber of $Z_{0}$ over $\left(L_{D}, L_{E}\right)$ is nonempty then we may choose a discrete valuation ring with uniformizer $t$ whose general point lies above the complement of $\Delta_{q} \cup \Delta_{r}$ and whose special point lies in $Z_{0}$. Let $b$ and $a$ be the orders of tangency to $\Delta_{q}$ and $\Delta_{r}$. Then $L \otimes \mathcal{O}_{C}\left(n p^{\prime}-n p\right)$ restricts to an invertible sheaf associated to a Cartier divisor $\alpha D+\beta E$ supported on the special fiber of $C_{T}$. Since $\alpha D+\beta E$ is a Cartier divisor, there must be $k, k^{\prime}, \ell, \ell^{\prime}, c, c^{\prime} i n \mathbf{Z}$ such that $\alpha=b k+c=a k^{\prime}+c^{\prime}$ and $\beta=b \ell+c=a \ell^{\prime}+c^{\prime}$ (corresponding to 
local equations $x^{k} y^{\ell} t^{c}$ for $\alpha D$ and $z^{k^{\prime}} w^{\ell^{\prime}} t^{c^{\prime}}$ for $\left.\beta E\right)$. These equations imply that $a(k-\ell)=b\left(\ell^{\prime}-k^{\prime}\right)$. We calculate $L_{D}$ and $L_{E}$ :

$$
\begin{aligned}
& L_{D}=\mathcal{O}_{D}\left(n p+(\ell-k) q+\left(\ell^{\prime}-k^{\prime}\right) r\right) \\
& L_{E}=\mathcal{O}_{D}\left(-n p^{\prime}+(k-\ell) q+\left(k^{\prime}-\ell^{\prime}\right) r\right)
\end{aligned}
$$

Note that $k-\ell$ and $k^{\prime}-\ell^{\prime}$ have the same sign, because both signs are the same as the sign of $\alpha-\beta$. Since $L_{D}$ and $L_{E}$ both have degree zero, we have $n=(k-\ell)+\left(k^{\prime}-\ell^{\prime}\right)$.

Conversely, Let $T$ be a curve in $S$ passing through $S_{0}$, tangent to $\Delta_{q}$ with order $b$, and tangent to $\Delta_{r}$ with order $a$. Let $t$ be a uniformizing parameter for $T$ at $S_{0}$. In local coordinates near $q$ on $C_{T}=C \times_{S} T$, we have $x y=t^{b}$ and near $r$ we have $z w=t^{a}$. Then the local equations $x^{a}=0$ and $z^{b}=0$ both describe the same Cartier divisor $a b D$ on the total space of $C_{T}$, and $\mathcal{O}_{E}(a b D)=\mathcal{O}_{E}(a q+b r)$. It follows that $\mathcal{O}_{C_{T}}\left(n p-n p^{\prime}+a b D\right)$ restricts to $\mathcal{O}_{D}(n p-a q-b r)$ on $D$ and to $\mathcal{O}_{E}\left(-n p^{\prime}+a q+b r\right)$ on $E$. This shows that $\left(L_{D}, L_{E}\right)$ is the limit of a curve that lies generically in the complement of $\Delta_{q}$ and $\Delta_{r}$ if $L_{D}$ and $L_{E}$ have the form required in the statement of the lemma.

Now we set $\mathbf{a}=(n,-n)$ and analyze the fiber of $\operatorname{Div}_{g, \mathbf{a}}$ over $S$. For simplicity, we focus on the multidegree 0 part of $\mathbf{D i v} g, \mathbf{a}$ (that is, the part of $\mathbf{D i v}{ }_{g, \mathbf{a}}$ whose image in $\mathbf{P i c} \mathbf{c}_{g, n}$ has multidegree 0), which we denote $\operatorname{Div}_{g, \mathbf{a}}^{0}$.

Let $M_{S}$ be the minimal logarithmic structure on $S$ associated to the family of curves $C$ over $S$. Its rank at $S_{0}$ is 2 . The fiber of $\mathbf{D i v}_{g, \mathbf{a}}$ over $S_{0}$ parameterizes quotient logarithmic structures $M_{S_{0}}^{\prime}$ of $M_{S_{0}}$, together with piecewise linear functions $\alpha$ on the dual graph of $C_{0}$ taking values in $\bar{M}_{S_{0}}^{\text {gp }}$. We denote this fiber $W$.

Such a piecewise linear function has integral slopes $a$ and $b$ on the edges corresponding to the nodes $q$ and $r$. These integers must both have the same sign if either is nonzero. We can compute that

$$
\left.\mathcal{O}_{C}(\alpha)\right|_{D}=\mathcal{O}_{D}(n p-a q-b r)
$$

where we have oriented the edges of the dual graph from $E$ to $D$. The integers $a$ and $b$ are locally constant on $W$, so $W$ has components indexed by partitions of $n$ into nonzero integers $a$ and $b$ of the same sign. The proof of Lemma 4.3.3.2 shows that each of these components maps isomorphically to $Z_{0}$.

Note that $\mathbf{D i v}_{g, \mathbf{a}}^{0}(C) \rightarrow Z \subset \mathbf{P i c}_{g, 2}^{0}(C)$ is finite and birational onto its image, but in many situations (depending on $g, n$, and the locations of $p, p^{\prime}, q$, and $r$ ) it is not an isomorphism. By Zariski's "Main Theorem," this means that $Z$ is not normal in these cases.

4.5. Logarithmic trivializations of line bundles. We saw in Theorem 4.3.2 that $\mathbf{D i v}_{g, \mathbf{a}} \rightarrow \mathbf{P i c}_{g, n}$ is a finite map. Therefore, if $S \rightarrow \mathbf{P i c}{ }_{g, n}$ classifies a line bundle $L$ on a logarithmic curve $C$ over $S$, then $S \times \mathbf{P i c}_{g, n} \mathbf{D i v}_{g, \text { a }}$ is representable by a finite map to $S$ whose fiber over a geometric point $s$ consists of the piecewise linear functions on the tropicalization of $C_{s}$ whose associated line bundle is $\left.L\right|_{C_{s}}$. In this section, we want to give a somewhat more intrinsic characterization of this space.

Suppose that $\mathbf{G}_{m}$ acts on $Y$ and that $L$ is a line bundle with associated $\mathbf{G}_{m}$-torsor $L^{*}$; that is, $L^{*}$ is the sheaf of isomorphisms $\mathcal{O} \rightarrow L$ and $L=\left(L^{*} \times \mathcal{O}\right) / \mathbf{G}_{m}$, where the action is antidiagonal. We denote by $L^{*} \otimes Y=\left(L^{*} \times Y\right) / \mathbf{G}_{m}$ the contraction of $L^{*}$ with $Y$. Thus $L=L^{*} \otimes \mathcal{O}$.

Recall from Section 3.1 that, given $\alpha \in \pi_{*}\left(\bar{M}_{C}^{\mathrm{gp}}\right)$ on a logarithmic curve $\pi: C \rightarrow S$ there is a canonical line bundle $\mathcal{O}_{C}(-\alpha)$ on $C$ associated to $\alpha$ given by taking the line bundle associated to the $\mathbf{G}_{m}$-torsor $\mathcal{O}_{C}^{*}(-\alpha)$ produced by the preimage of $\alpha$ through the quotient map $M_{C}^{\mathrm{gp}} \rightarrow \bar{M}_{C}^{\mathrm{gp}}$. If $\alpha$ is instead a logarithmic divisor in $\pi_{*}\left(\bar{M}_{C}^{\mathrm{gp}}\right) / \bar{M}_{S}^{\mathrm{gp}}$ then by choosing a representative in $\pi_{*} \bar{M}_{C}^{\mathrm{gp}}$, we obtain a line bundle that is well defined up to tensor product by $\mathcal{O}_{C}(\delta)$ for $\delta \in \bar{M}_{S}^{\text {gp }}$.

Definition 4.5.1. Let $\pi: C \rightarrow S$ be a logarithmic curve and let $L$ be a line bundle on $C$. A logarithmic trivialization of $L$ over $S$ is a section of the quotient $\pi_{*}\left(L^{*} \otimes M_{C}^{\mathrm{gp}}\right) / M_{S}^{\mathrm{gp}}$.

Let $C \rightarrow S$ be a fixed family of $n$-marked logarithmic curves over $S$, fix an equivalence class of line bundles $L$ on $C$, up to tensor product by line bundles from the base, and fix a vector a of integer weights.

Definition 4.5.2. We denote by $\operatorname{Div}_{g, \mathbf{a}}(C, L)$ the category whose objects are:

(1) a logarithmic scheme $S^{\prime}$ and map $f: S^{\prime} \rightarrow S$, with $C^{\prime}=f^{-1} C$, and

(2) a logarithmic trivialization of $f^{*} L$.

This naturally forms a category fibered in groupoids over LogSch. 
Proposition 4.5.3. There is a cartesian diagram:

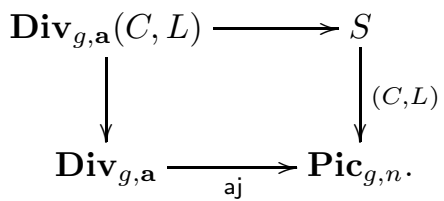

In particular, $\operatorname{Div}_{g, \mathbf{a}}(C, L)$ is proper over $S$.

Proof. Note that $L^{*} \otimes M_{C}^{\mathrm{gp}}$ is an $\mathcal{O}_{C}^{*}$-torsor over $\bar{M}_{C}^{\mathrm{gp}}$. We regard this as an exact sequence of pointed stacks:

$$
0 \rightarrow L^{*} \otimes M_{C}^{\mathrm{gp}} \rightarrow \bar{M}_{C}^{\mathrm{gp}} \rightarrow \mathrm{BO}_{C}^{*}
$$

Pushing forward by $\pi_{*}$ gives an exact sequence of pointed sets (4.5.3.1), as in (4.3.1.1):

$$
0 \rightarrow \pi_{*}\left(L \otimes M_{C}^{\mathrm{gp}}\right) \rightarrow \pi_{*} \bar{M}_{C}^{\mathrm{gp}} \rightarrow \pi_{*} \mathrm{~B} \mathcal{O}_{C}^{*}
$$

Dividing by the sequence

$$
0 \rightarrow M_{S}^{\mathrm{gp}} \rightarrow \bar{M}_{S}^{\mathrm{gp}} \rightarrow \mathrm{BO}_{S}^{*} \rightarrow 0
$$

and noting that $\pi_{*}\left(\mathrm{BO}_{C}^{*}\right) / B \mathcal{O}_{S}^{*}=R^{1} \pi_{*} \mathcal{O}_{C}^{*}$, we obtain another exact sequence

$$
0 \rightarrow \pi_{*}\left(L \otimes M_{C}^{\mathrm{gp}}\right) / M_{S}^{\mathrm{gp}} \rightarrow \pi_{*}\left(\bar{M}_{C}^{\mathrm{gp}}\right) / \bar{M}_{S}^{\mathrm{gp}} \rightarrow R^{1} \pi_{*} \mathcal{O}_{C}^{*}
$$

Here, the map from $\pi_{*}\left(\bar{M}_{C}^{\mathrm{gp}}\right) / \bar{M}_{S}^{\mathrm{gp}} \rightarrow R^{1} \pi_{*} \mathcal{O}_{C}^{*}$ sends the equivalence class of $\alpha \in \pi_{*} \bar{M}_{C}^{\mathrm{gp}}$ to $L(-\alpha)$. The sequence therefore identifies $\pi_{*}\left(L \otimes M_{C}^{\mathrm{gp}}\right) / M_{S}^{\mathrm{gp}}$ - that is, $\operatorname{Div}_{g, \mathbf{a}}(C, L)$ - with the the $\alpha \in \operatorname{Div}_{g, \mathbf{a}}(C)$ whose image in $\mathbf{P i c} \mathbf{c}_{g, n}$ is $L$. This is the content of the cartesian diagram in the statement of the proposition.

This proposition can be applied with $S=\mathfrak{M}_{g, n}^{\log }$, with $C$ taken to be the universal curve, and with $L=\omega_{C / S}^{k}$. Then $\operatorname{Div}_{g, n}\left(C, \omega_{C / S}^{k}\right)$ is a compactification of the moduli space of $k$-differentials on curves, and we will see in Section 4.6 that it is equipped with a virtual fundamental class. In compact type, aj is the usual Abel-Jacobi map and the virtual fundamental class of $\operatorname{Div}(X, L)$ restricts to the refined intersection of the Abel-Jacobi section and the section determined by $L$.

4.6. Twisted double ramification cycle. The composition of the sequence of morphisms (4.6.1)

$$
\operatorname{Div}_{g, \mathbf{a}} \stackrel{\text { aj }}{\rightarrow} \mathbf{P i c}_{g, n} \stackrel{\Pi}{\rightarrow} \mathfrak{M}_{g, n}^{\log }
$$

is logarithmically étale, the map aj is a closed embedding, and the map $\Pi$ is strict (by definition of the logarithmic structure on $\mathbf{P i c} \mathbf{c}_{g, n}$ ) and smooth. This implies that $\mathbf{P i c} \mathbf{c}_{g, n} \times_{\mathfrak{M}_{g, n}^{\log }} \mathbf{D i v} \mathbf{i v}_{g, \mathbf{a}}$ is strict and smooth over $\operatorname{Div}_{g, \mathbf{a}}$, and the induced section $\sigma$ is therefore a local complete intersection embedding. Writing $I$ for the sheaf of ideals of $\sigma$, we therefore have an isomorphism

$$
\text { aj }^{*} I=\text { aj }^{*} \Omega_{\mathbf{P i c}_{g, n} / \mathfrak{M}_{g, n}^{\log }} .
$$

Furthermore aj $^{*} I[1]$ can serve as the obstruction bundle of a relative obstruction theory for $\sigma$ (we may identify $\mathrm{aj}^{*} I[1]$ with the relative cotangent complex of $\sigma$ ). Since

$$
\mathbf{P i c}_{g, n} \times \underset{\mathfrak{M}_{g, n} \times \operatorname{Div}_{g, \mathbf{a}}}{\operatorname{lig}_{g}} \rightarrow \mathbf{P i c}_{g, n}
$$

is the base change of a logarithmically étale morphism, it is also logarithmically étale over $\mathbf{P i c}_{g, n}$. Therefore our obstruction theory also works as a relative logarithmic obstruction theory for aj : $\mathbf{D i v}_{g, \mathbf{a}} \rightarrow \mathbf{P i c}_{g, n}$.

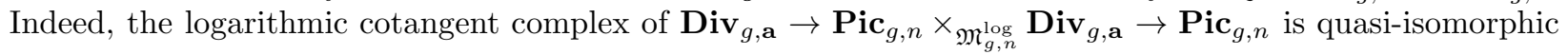
to the relative cotangent complex of $\mathbf{D i v} g_{g, \mathbf{a}} \rightarrow \mathbf{P i c}_{g, n}$ by the transitivity triangle [Ols05, 1.1 (v) and Remark 3.14] (note that logarithmically étale morphisms are logarithmically flat).

It is also well-known that the relative tangent bundle of $\mathbf{P i c}_{g, n}$ over $\mathfrak{M}_{g, n}^{\log }$ has fiber $H^{1}\left(C, \mathcal{O}_{C}\right)$ over the point corresponding to a logarithmic curve $C$. This proves Theorem B.

It follows that, for any $f: S \rightarrow \mathbf{P i c}_{g, n}$ classifying a logarithmic curve $C$ over $S$ and line bundle $L$ on $C$, the pullback $\operatorname{Div}(C, L)$ of aj: $\operatorname{Div}_{g, \mathbf{a}} \rightarrow \mathbf{P i c}_{g, n}$ has a perfect relative obstruction theory $f^{*}$ aj $^{*} I$. Let $S$ be an algebraic stack with a logarithmic structure, let $C$ be a logarithmic curve of genus $g$ over $S$, and let $L$ 
be a line bundle on $C$. These data give a morphism $S \rightarrow \mathbf{P i c}_{g, n}$. Fix an $n$-tuple of integers $\mathbf{a}$. We have (Section 4.5)

$$
\operatorname{Div}_{g, \mathbf{a}}(C, L)=S \underset{\mathbf{P i c}_{g, n}}{\operatorname{Div}_{g, \mathbf{a}}}
$$

Gysin pullback for the Abel-Jacobi section equips the map $\operatorname{Div}_{g, \mathbf{a}}(C, L) \rightarrow S$ with a virtual pullback. In particular, if $S$ has a fundamental class $[S]$ (or a virtual fundamental class) then we obtain a relative virtual fundamental class $\left[\operatorname{Div}_{g, \mathbf{a}}(C, L) / S\right]^{\text {vir }}$ by applying the virtual pullback to $[S]$.

We can apply this in particular where $S=\overline{\mathcal{M}}_{g, n}$, where $C$ is the universal curve, and where $L$ is $\omega_{C / S}^{k}$ for any integer $k$. This gives a cycle class

$$
\left[\operatorname{Div}_{g, \mathbf{a}}\left(\mathcal{M}_{g, n}^{\log }, \omega^{k}\right)\right]^{\mathrm{vir}}
$$

in $\mathrm{CH}_{2 g-3+n}\left(\mathbf{D i v}_{g, \mathbf{a}}\left(\mathcal{M}_{g, n}^{\log }, \omega^{k}\right)\right)$. Since $\mathbf{D i v}_{g, \mathbf{a}}\left(\omega^{\otimes k}\right)$ is proper over $\overline{\mathcal{M}}_{g, n}$, we may push this cycle class forward to $\overline{\mathcal{M}}_{g, n}$ to obtain a twisted double ramification cycle $\operatorname{DR}_{g, \mathbf{a}}\left(\mathcal{M}_{g, n}^{\log }, \omega^{k}\right)$.

4.7. The Abel-Jacobi map. Here we construct a proper map $\mathbf{D i v}_{g, \mathbf{a}} \rightarrow \mathbf{P i c}_{g}$ extending the map $\mathcal{M}_{g, \mathbf{a}} \rightarrow$ $\mathbf{P i c}_{g}$ that sends a smooth curve $C$ with $n$ distinct marked points $x_{1}, \ldots, x_{n}$ to $\mathcal{O}_{C}\left(\sum a_{i} x_{i}\right)$.

Suppose that $C$ is a logarithmic curve without markings. By a punctured model of $C$ we will mean a map $\tau: \tilde{C} \rightarrow C$ such that, if the markings of $\tilde{C}$ are forgotten, the stabilization of $\tau$ is an isomorphism. We call it a stable punctured model if the underlying curve $\tilde{C}$ is stable. If $\mathfrak{C}$ is a tropical curve and $\alpha$ is a piecewise linear function on $\mathfrak{C}$ that is linear on the edges, we call $\alpha$ linear at a vertex of $\mathfrak{C}$ if the sum of the outgoing slopes of $\alpha$ at that vertex is zero. We apply the same terminology to logarithmic divisors.

Let $\mathbf{D i v}_{g, \mathbf{a}}^{*}$ be the stack over logarithmic schemes whose $S$-points are the following data:

(1) a logarithmic curve $C$ over $S$ without marked points,

(2) a stable punctured model $\tau: \tilde{C} \rightarrow C$ with $n$ marked points, and

(3) a logarithmic divisor $\alpha: \tilde{C} \rightarrow \mathcal{P}$ and slopes a on its legs, such that

(4) $\alpha$ is linear at the components of $\tilde{C}$ collapsed by $\tau$.

Let us also denote by $\mathbf{P i c}{ }_{g, n}^{*}$ the stack over logarithmic schemes whose $S$-points are

(1) a logarithmic curve $C$ over $S$ without marked points,

(2) a stable punctured model $\tau: \tilde{C} \rightarrow C$ with $n$ marked points, and

(3) an equivalence class of line bundles $\tilde{L}$ on $\tilde{C}$, up to tensor product by line bundles from the base, such that

(4) $L$ has degree zero on all components collapsed by $\tau$.

If $\alpha: \tilde{C} \rightarrow \mathcal{P}$ is an object of $\mathbf{D i v}_{g, \mathbf{a}}^{*}$ then $\mathcal{O}_{\tilde{C}}(\alpha)$ is an object of $\mathbf{P} \mathbf{c}_{g, n}^{*}$. By Theorem 4.3.2, we have a proper map:

$$
\operatorname{Div}_{g, \mathbf{a}}^{*} \rightarrow \mathbf{P i c}_{g, n}^{*} .
$$

On the other hand, if $(\tau: \tilde{C} \rightarrow C, \tilde{L})$ is an object of $\mathbf{P i c}_{g, n}^{*}$ then $\tilde{L}$ is the pullback to $\tilde{C}$ of the line bundle $\tau_{*} \tilde{L}$ on $C$. Therefore $\mathbf{P i c}_{g, n}^{*}$ is equivalent to the space of

(1) a logarithmic curve $C$ over $S$ without marked points,

(2) a stable punctured model $\tau: \tilde{C} \rightarrow C$ with $n$ marked points, and

(3) an equivalence class of line bundles $L$ on $C$.

That is, $\mathbf{P i c}{ }_{g, n}^{*} \rightarrow \mathbf{P i c} c_{g}$ is proper, with the preimage of $(C, L)$ corresponding to space of punctured models of $C$ (equivalently, the space of stable curves that stabilize to $C$ when their marked points are forgotten).

\section{RUBBER COMES FROM THE TROPICS}

\subsection{Aligned logarithmic curves.}

Definition 5.1.1. Let $C$ be a logarithmic curve over $S$ and let $\alpha: C \rightarrow \mathcal{P}$ be a logarithmic divisor on $C$. We say that $\alpha$ is aligned if, for each geometric point $s$ of $S$, the set of $\alpha(v)$, as $v$ ranges among the vertices of $C_{s}$, is totally ordered. We write Rub for the subfunctor of Div whose objects are aligned logarithmic divisors.

Proposition 5.1.2. Rub is a logarithmic modification of Div, and in particular is representable by an algebraic stack with a logarithmic structure. 
Proof. It is sufficient to show that $\mathbf{R u b} \times_{\mathbf{D i v}} S$ is a logarithmic modification for each logarithmic scheme $S$ and map $S \rightarrow$ Div. In other words, we must show that if $S$ is a logarithmic scheme, $C$ is a logarithmic curve over $S$, and $\alpha: C \rightarrow \mathcal{P}$ is a logarithmic divisor on $C$, then the space of alignments of $\alpha$ is a logarithmic modification of $S$.

This is a local assertion in the strict étale topology on $S$, so we can assume that there is a geometric point $s$ of $S$ such that

$$
\Gamma\left(S, \bar{M}_{S}\right) \rightarrow \bar{M}_{S, s}
$$

is a bijection and that the family of dual graphs of the fibers of $C$ over the closed stratum of $S$ has trivial monodromy.

Let $\Gamma$ be the tropicalization of $C_{s}$. For each vertex $v$ of $\Gamma$, we get a value $\alpha(v) \in \bar{M}_{S}^{\mathrm{gp}}$, and $\alpha$ is aligned if and only if these elements are totally ordered.

Passing to an étale cover of $S$, we can assume that all of the $\alpha(v)$ are pulled back along some map from $S$ to a toric variety $V$. The space of alignments of $\alpha$ is also pulled back from $V$, where it is easily seen to be representable by a toric modification: subdivide the cone of $V$ along the hyperplanes $\alpha(v)=\alpha(w)$.

As a special case of the constructions in this section and as motivation for what's to come, we identify the fiber product

$$
\mathbf{R u b}_{g, \mathbf{a}}\left(\mathcal{M}_{g, n}^{\log }, \mathcal{O}\right)=\mathbf{R u b}_{g, \mathbf{a}} \underset{\mathbf{P i c}_{g, n}}{\times} \mathcal{M}_{g, n}^{\log }
$$

where $\mathcal{M}_{g, n}^{\log }$ is the moduli space of stable logarithmic curves and the map $\mathcal{M}_{g, n}^{\log } \rightarrow$ Pic $_{g, n}$ sends a curve to the trivial line bundle on that curve. The following theorem will be demonstrated in Section 5.5.

Theorem 5.1.3. $\operatorname{Rub}\left(\mathcal{M}_{g, n}^{\log }, \mathcal{O}\right)$ is the moduli space of predeformable maps from prestable curves to rubber targets. The stable locus is the space of stable maps to rubber targets introduced by Graber and Vakil [GV05].

\subsection{Divided tropical lines.}

Definition 5.2.1. Let $S$ be a logarithmic scheme. A divided tropical line bundle over $S$ is a tropical line bundle $\mathcal{P}$ and a subfunctor $\mathcal{Q} \subset \mathcal{P}$ such that, locally in $S$, there are sections $\gamma_{0} \leq \cdots \leq \gamma_{n}$ of $\mathcal{P}$ and $\mathcal{Q}$ is the set of sections of $\mathcal{P}$ that are comparable to all of the $\gamma_{i}$. Given any tropical line $\mathcal{P}$, we write $\mathcal{P}$ for the divided tropical line over $S$ associated to a sequence $\gamma_{0} \leq \cdots \leq \gamma_{n}$ of $\mathcal{P}$. If $\mathcal{P}$ is the tropical line bundle associated to a logarithmic line bundle $P$ then we also write $P_{\gamma}=\mathcal{P}_{\gamma} \times_{\mathcal{P}} P$.

Example 5.2.2. Over any base $S$, we may take $P=\mathbf{G}_{m}^{\log }, \mathcal{P}=\mathbf{G}_{m}^{\text {trop }}$, and $\gamma_{0}=0$ to get a divided tropical line $\mathcal{P}_{\gamma}$ and a divided logarithmic line $P_{\gamma}$. In this case $P_{\gamma}=\mathbf{P}^{1}$, with its standard logarithmic structure, and $\mathcal{P}_{\gamma}=\left[\mathbf{P}^{1} / \mathbf{G}_{m}\right]$.

If $\mathcal{P}_{\gamma}$ is a divided tropical line over $S$, the sequence $\gamma_{0} \leq \cdots \leq \gamma_{n}$ cannot be recovered from $\mathcal{P}_{\gamma}$. However, we will show that the differences $\delta_{i}=\gamma_{i+1}-\gamma_{i}$ can (see Figure 2).

Consider the locus $U_{i} \subset \mathcal{P}_{\gamma}$ consisting of those $\alpha$ such that $\gamma_{i} \leq \alpha \leq \gamma_{i+1}$. This is an open subfunctor of $\mathcal{P}_{\gamma}$. Let $\beta=\alpha-\gamma_{i}$ and let $\beta^{\prime}=\gamma_{i+1}-\alpha$. Then $\beta+\beta^{\prime}=\delta_{i}$, which means that

$$
U_{i}(T) \simeq\left\{\left(\beta, \beta^{\prime}\right) \in \Gamma\left(T, \bar{M}_{T}\right) \times \Gamma\left(T, \bar{M}_{T}\right) \mid \beta+\beta^{\prime}=\delta_{i}\right\}
$$

functorially in $T$. It follows from this that the minimal logarithmic structure of $S$ over which $\mathcal{P}_{\gamma}$ is defined is freely generated by the nonzero $\delta_{i}$. (If $\delta_{i}=0$, then $U_{i}$ is an open subset of $U_{i+1}$ and $U_{i-1}$, so it can be omitted from discussion.)

We argue that the order of the $\delta_{i}$ is also determined by $\mathcal{P}_{\gamma}$. Indeed, $U_{i} \cap U_{j} \neq \varnothing$ if and only if $\gamma_{i+1}=\gamma_{j}$ or $\gamma_{j+1}=\gamma_{i}$. It is not possible for both to occur unless $\gamma_{i}=\gamma_{i+1}=\gamma_{j}=\gamma_{j+1}$, in which case $\delta_{i}=\delta_{j}=0$. Otherwise, we can recognize that $i \leq j$ if $\gamma_{i+1}=\gamma_{j}$ and that $j \leq i$ if $\gamma_{i}=\gamma_{j+1}$.

We also observe that if $\mathcal{Q}$ is a divided tropical line with lengths $\delta_{1}, \ldots, \delta_{n}$ and we set

$$
\begin{gathered}
\gamma_{i}=\sum_{j \leq i} \delta_{j} \\
\gamma_{i}^{\prime}=-\sum_{j \geq i} \delta_{j}
\end{gathered}
$$

then $\mathcal{Q} \simeq \mathcal{P}_{\gamma} \simeq \mathcal{P}_{\gamma^{\prime}}$ where $\mathcal{P}=\mathbf{G}_{m}^{\text {trop }}$. In other words, the tropical line bundle underlying a divided tropical line bundle has two canonical trivializations, corresponding to the smallest and largest vertices of the subdivision. 


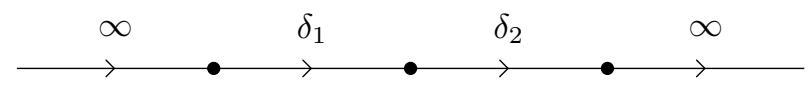

Figure 2. A divided tropical line with $\gamma_{2}=\gamma_{1}+\delta_{1}$ and $\gamma_{3}=\gamma_{2}+\delta_{2}$.

Remark 5.2.3. Minimal tropical lines with at least one division were called aligned logarithmic structures in [ACFW13], where they were studied in terms of the sequence $\delta_{1}, \ldots, \delta_{n}$ and the condition (5.2.1):

$$
0 \leq \delta_{1} \leq \delta_{1}+\delta_{2} \leq \cdots \leq \delta_{1}+\cdots+\delta_{n} .
$$

Proposition 5.2.4. Let $\mathcal{P}$ be a tropical line. If $\gamma=\left\{\gamma_{1} \leq \cdots \leq \gamma_{n}\right\}$ is a nonempty collection of sections of $\mathcal{P}$ then $\mathcal{P}_{\gamma}$ is representable by an algebraic stack over $S$ with a logarithmic structure and $P_{\gamma}$ is representable by a family of 2-marked, semistable, genus 0 curves.

Proof. The assertion is local in $S$, so we may assume $\mathcal{P} \simeq \mathbf{G}_{m}^{\text {trop }}$.

We have $P_{\gamma}=\mathcal{P}_{\gamma} \times{ }_{\mathbf{G}_{m}^{\text {trop }}} \mathbf{G}_{m}^{\text {log }}$. We will check that $P_{\gamma}$ is a family of 2-marked semistable curves over $S$. The assertion about $\mathcal{P}_{\gamma}$ follows, since $\mathcal{P}_{\gamma}=\left[P_{\gamma} / \mathbf{G}_{m}\right]$.

The assertions of the proposition are local in $S$, so we may assume that the $\gamma_{i}$ lift to elements $\tilde{\gamma}_{i} \in$ $\Gamma\left(S, M_{S}^{\mathrm{gp}}\right)$. Then a morphism $X \rightarrow P_{\gamma}$ consists of a morphism $f: X \rightarrow S$ and $\tilde{\alpha} \in \Gamma\left(X, M_{X}^{\mathrm{gp}}\right)$ with image $\alpha \in \Gamma\left(X, \bar{M}_{X}^{\text {gp }}\right)$ such that $\alpha$ is comparable to every $\gamma_{i}$.

We argue first that $P_{\gamma}$ is a flat family of nodal curves. Consider the locus $U_{i} \subset P_{\gamma}$ where $\gamma_{i} \leq \alpha \leq \gamma_{i+1}$. Taking $\tilde{\delta}_{i}=\tilde{\gamma}_{i+1} \tilde{\gamma}_{i}^{-1}$, we can identify $U_{i}(X)$ with the locus of pairs $\left(\tilde{\beta}, \tilde{\beta}^{\prime}\right) \in \Gamma\left(X, M_{X}^{\mathrm{gp}}\right)$ such that $\tilde{\beta} \tilde{\beta}^{\prime}=\tilde{\delta}_{i}$. In other words, there is a cartesian diagram of logarithmic schemes:

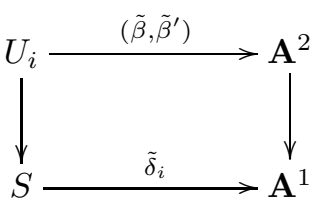

where the map $\mathbf{A}^{2} \rightarrow \mathbf{A}^{1}$ is multiplication. This proves $U_{i}$ is representable by a logarithmic scheme, flat over $S$, and has nodal curves as its fibers.

At the extremes, $i=0$ and $i=n$, a similar argument gives $U_{i} \simeq \mathbf{A}_{S}^{1}$.

Now we argue that $P_{\gamma}$ is proper. For this we may assume that $S$ is the spectrum of a valuation ring with generic point $\eta$, that $S$ has the maximal logarithmic structure extending the logarithmic structure from $\eta$, and that we are given a section of $P_{\gamma}$ over $\eta$. That is, we are given an element $\tilde{\alpha}$ of $\Gamma\left(\eta, M_{\eta}^{\mathrm{gp}}\right)$ that is comparable to the $\tilde{\gamma}_{i}$. We wish to show that this extends uniquely to $\Gamma\left(S, M_{S}^{\mathrm{gp}}\right)$. But by construction, $\Gamma\left(\eta, M_{\eta}^{\mathrm{gp}}\right)=\Gamma\left(S, M_{S}^{\mathrm{gp}}\right)$, so we obtain the lift of $\tilde{\alpha}$ automatically. The comparability to the $\tilde{\gamma}_{i}$ is not as immediate, because the partial order on $M_{S}^{\mathrm{gp}}$ has fewer relations than does $M_{\eta}^{\mathrm{gp}}$. However, because $M_{S}$ is the maximal extension of $M_{\eta}$, any two elements of $\bar{M}_{S}^{\mathrm{gp}}$ whose images are comparable in $\bar{M}_{\eta}^{\mathrm{gp}}$ are also comparable in $\bar{M}_{S}$ (Proposition 3.5.5). This completes the proof of properness.

Finally, we check that the fibers of $P_{\gamma}$ are semistable, 2-marked curves. We may assume $S$ is the spectrum of an algebraically closed field. Let $V_{i}$ be the locus in $P_{\gamma}$ where $\alpha=\gamma_{i}$. Then we combine

$$
P_{\gamma}=U_{0} \underset{V_{1}}{\cup} U_{1} \underset{V_{2}}{\cup} U_{2} \underset{V_{3}}{\cup} \cdots \underset{V_{n-1}}{\cup} U_{n-1} \underset{V_{n}}{\cup} U_{n}
$$

with the identifications

$$
\begin{aligned}
U_{0} & \simeq \mathbf{A}_{S}^{1} \\
U_{i} & \simeq S_{\mathbf{A}^{2}} \mathbf{A}^{1} \\
U_{n} & \simeq \mathbf{A}^{1}
\end{aligned}
$$

and a quick check of the gluing to confirm that $P_{\gamma}$ is a 2-marked, semistable curve.

The proposition shows that any tropical line $\mathcal{P}_{\gamma}$ over $S$ with at least one division gives rise to a family of 2-marked, semistable, rational curves $P_{\gamma}$. Thus $\gamma$ determines a morphism $S \rightarrow \mathfrak{M}_{0,2}^{\text {ss }}$. Conversely, if $P$ is such 
a family of curves over a logarithmic scheme $S$, then locally $P$ has smoothing parameters $\delta_{1}, \delta_{2}, \ldots, \delta_{n} \in$ $\Gamma\left(S, \bar{M}_{S}\right)$ associated to its nodes. The sequence of elements

$$
\gamma_{i}=\sum_{j \leq i} \delta_{i}
$$

is then a divided tropical line over $S$.

These two operations are not quite inverse to one another because the family of rational curves carries a $\mathbf{G}_{m}$ automorphism that is not visible in the tropical line. It is shown that in [ACFW13] that when this automorphism is rigidified away, $\mathfrak{M}_{0,2}^{\mathrm{ss}}$ is indeed the moduli space of tropical lines with at least one division.

Theorem 5.2.5. There is an algebraic stack $\mathscr{T}$ with logarithmic structure parameterizing families of tropical lines with at least one division, and $\mathfrak{M}_{0,2}^{\mathrm{ss}} \simeq \mathscr{T} \times \mathrm{BG}_{m}$.

We sketch the argument using the terminology of this paper. Suppose that $P$ is a family of 2 -marked, semistable, genus 0 curves over $S$. If $s$ is a geometric point of $S$ and $P_{s}$ has $n$ nodes then $\bar{M}_{S, s}$ contains smoothing parameters for those nodes $\delta_{1}, \ldots, \delta_{n}$; because $P_{s}$ is a chain of rational curves, the $\delta_{i}$ have a canonical order, with the indices corresponding to proximity to the first marked point. As we have seen above, the $\delta_{i}$ give a divided tropical line over $S$.

We can also extract a line bundle from $P$ by taking the normal bundle at the first marked point. Together, these give a map:

$$
\mathfrak{M}_{0,2}^{\mathrm{ss}} \rightarrow \mathscr{T} \times \mathrm{BG}_{m}
$$

Conversely, if $\mathcal{P}_{\gamma}$ is a divided tropical line over $S$ then $P_{\gamma}$ is a family of 2-marked, genus 0 semistable curves by Proposition 5.2.4. Twisting by $L$, the family $L \otimes P_{\gamma}$ is also a chain of rational curves. The map that sends $\left(\mathcal{P}_{\gamma}, L\right)$ to $L \otimes P_{\gamma}$ is inverse to (5.2.5.1).

We note that we could have used the normal bundle at the second marked point in the discussion above and obtained a second identification between $\mathfrak{M}_{0,2}^{\mathrm{ss}}$ and $\mathscr{T} \times \mathbf{B G}_{m}$. These identifications can be related by a formula involving the $\delta_{i}$.

5.3. Rubber divisors. Suppose that $C$ is a logarithmic curve over $S$ and that $\alpha: C \rightarrow \mathcal{P}$ is a logarithmic divisor on $C$. For each irreducible component $v$ of each geometric fiber of $C$ over $S$, the restriction of $\alpha$ to $v$ determines an element $\alpha(v) \in \bar{M}_{S}^{\mathrm{gp}}$, well-defined up to simultaneous shift of all $\alpha(v)$. As $\bar{M}_{S}^{\mathrm{gp}}$ is partially ordered (with $\bar{M}_{S}$ being the cone of elements $\geq 0$ ), this gives a partial order on the vertices of the dual graph of each fiber of $C$ over $S$. This partial order is compatible with generization in the evident sense: whenever $v$ and $w$ are irreducible components of $C_{s}$, generizing to irreducible components $v^{\prime}$ and $w^{\prime}$ of $C_{s^{\prime}}$, then $\alpha(v) \leq \alpha(w)$ implies $\alpha\left(v^{\prime}\right) \leq \alpha\left(w^{\prime}\right)$.

Definition 5.3.1. Let $\mathbf{R u b}_{g, n}$ be the subfunctor of $\operatorname{Div}_{g, n}$ parameterizing those pairs $(\pi: C \rightarrow S, \alpha)$ such that the values taken by $\alpha$ on the vertices of the dual graph of each geometric fiber of $C$ over $S$ are totally ordered in the partial order of $\bar{M}_{S}^{\mathrm{gp}}$. We call these logarithmic divisors linearly aligned.

Warning 5.3.2. Although $\mathbf{R u b}_{g, n}$ is a subset of $\mathbf{D i v}{ }_{g, n}$ when viewed as a functor on logarithmic schemes, that does not mean the underlying scheme of $\mathbf{R u b}_{g, n}$ is a subscheme of the underlying scheme of $\mathbf{D i v} \mathbf{v}_{g, n}$. Far from it: we will see shortly that the underlying algebraic stack of $\mathbf{R u b}_{g, n}$ is a birational modification of the underlying algebraic stack of $\operatorname{Div}_{g, n}$.

The following proposition gives a slightly simpler description of $\mathbf{R u b}_{g, n}$. Notably, it permits us to study points of $\mathbf{R u b}_{g, n}$ as sections of $\pi_{*}\left(\bar{M}_{C}^{\mathrm{gp}}\right)$ with a condition, instead of as sections of the quotient $\pi_{*}\left(\bar{M}_{C}^{\mathrm{gp}}\right) / \bar{M}_{S}^{\mathrm{gp}}$.

Proposition 5.3.3. Let $\mathbf{R u b}_{g, n}^{\prime}$ be the stack of tuples $(C, \alpha)$ with ordered marked points, where $C$ is a logarithmic curve over $S$ and $\alpha$ is a global section of $\bar{M}_{C}^{\mathrm{gp}}$, such that, for each geometric point $s$ of $S$, the values taken by $\alpha$ on the vertices of the dual graph of $C$ are totally ordered in $\bar{M}_{S, s}^{g p}$ and the minimal value taken is 0 . Then the natural map $\mathbf{R u b}_{g, n}^{\prime} \rightarrow \mathbf{R u b}_{g, n}$ is an isomorphism.

Proof. If $(C, \mathbf{x}, \alpha)$ is an object of $\mathbf{R u b}_{g, n}(S)$ then by definition, $\alpha$ is a section of $\pi_{*}\left(\bar{M}_{C}^{\mathrm{gp}}\right) / \bar{M}_{S}^{\mathrm{gp}}$. But because the values of $\alpha$ taken on the vertices of the tropicalization of $C$ are totally ordered, there is a unique representative of $\alpha$ in $\Gamma\left(C, \bar{M}_{C}^{\mathrm{gp}}\right)$ such that, in each fiber of $C$ over $S$, the smallest value it takes on the tropicalization is 0 . 


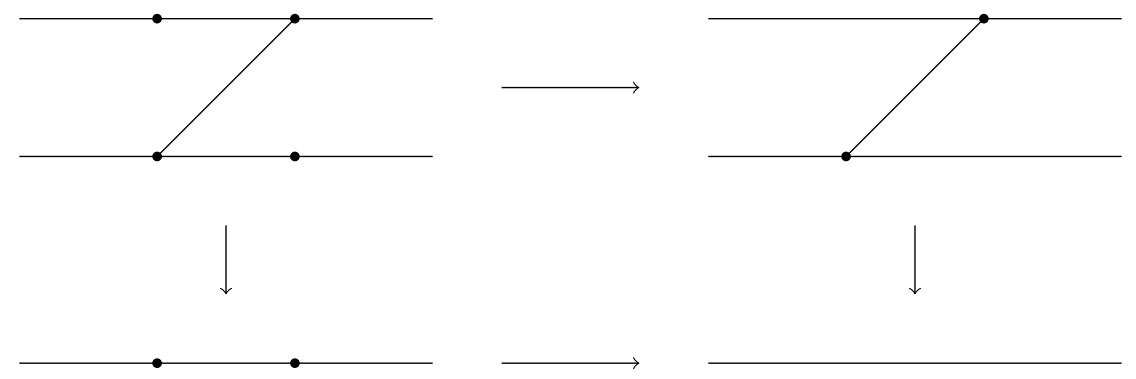

FiguRE 3. The tropicalization of a rubber morphism on the right side, with the induced subdivision of the tropical line in the lower left and the induced subdivision of the domain curve in the upper left

Theorem 5.3.4. The map $\mathbf{R u b}_{g, n} \rightarrow \mathbf{D i v}_{g, n}$ is a logarithmic modification.

Proof. We can represent an $S$-point of $\mathbf{D i v}_{g, n}$ locally as a logarithmic curve $C$ over $S$ and a piecewise linear function $\alpha$ on the tropicalization of $C$ taking values in $\bar{M}_{S}$. Localizing in $S$, we can lift these elements to $M_{S}$ so that they define a map to the toric variety $\mathbf{A}^{p}$. There is a minimal subdivision of the toric fan of $\mathbf{A}^{p}$ such that the $p$ coordinates are pairwise comparable. The corresponding toric modification of $\mathbf{A}^{p}$ pulls back under $S \rightarrow \mathbf{A}^{p}$ to $S \times \mathbf{D i v}_{g, n} \mathbf{R u b}_{g, n}$. Hence, $\mathbf{R u b}_{g, n}$ is locally the base change of a toric modification and is therefore a logarithmic modification, by definition.

Corollary 5.3.5. The map $\mathbf{R u b}_{g, n} \rightarrow \mathbf{D i v}_{g, n}$ is proper, logarithmically étale, birational, and a logarithmic monomorphism.

Proof. This is immediate from Theorem 5.3.4 and Proposition 3.4.2.

5.4. Divisions. Any morphism $S \rightarrow \mathbf{R u b}_{g, n}$ induces a divided tropical line on $S$ with at least one division. Indeed, if $(C, \alpha) \in \mathbf{R u b}_{g, n}(S)$ then locally $\alpha$ can be represented by a section $\Gamma\left(C, \bar{M}_{C}^{\text {gp }}\right)$. In fact, by Proposition 5.3.3, it is possible to choose $\alpha$ globally. By definition of $\mathbf{R u b}_{g, n}$, the family of elements $\alpha(v)$, as $v$ varies among vertices of the tropicalizations of the fibers of $C$ over $S$, is totally ordered in each fiber. Therefore the $\alpha(v)$ determine a divided tropical line $\mathcal{P}_{\gamma}$ over $S$. Note that although the $\alpha(v)$ are only well-defined up to addition of a constant from $\bar{M}_{S}^{\mathrm{gp}}$, the divided tropical line $\mathcal{P}_{\gamma}$ is independent of this ambiguity.

The map $C \rightarrow \mathbf{G}_{m}^{\text {trop }}$ classified by $\alpha$ does not necessarily factor through $\mathcal{P}_{\gamma}$. Figure 3 shows how this can happen at the level of tropical curves, where there is no arrow from the curve in the upper right to the divided tropical line in the lower left. However, $C \times{ }_{\mathbf{G}_{m}^{\text {trop }}} \mathcal{P}_{\gamma}$ is a logarithmic modification of $C$ whose morphism to $\mathbf{G}_{m}^{\text {trop }}$ does factor through $\mathcal{P}_{\gamma}$, by definition.

\subsection{Stable maps to rubber targets.}

Definition 5.5.1. Let $\pi: C \rightarrow S$ be family of logarithmic curves, and $L$ a line bundle on $C$. We define $\mathbf{R u b}_{g, \mathbf{a}}(C, L)$ to be the moduli space of logarithmic trivializations (Definition 4.5.1) of $L$ over $C$ whose image in $\mathbf{D i v}_{g, \mathbf{a}}$ lies in $\mathbf{R u b}_{g, \mathbf{a}}$.

By definition, there is a cartesian diagram:

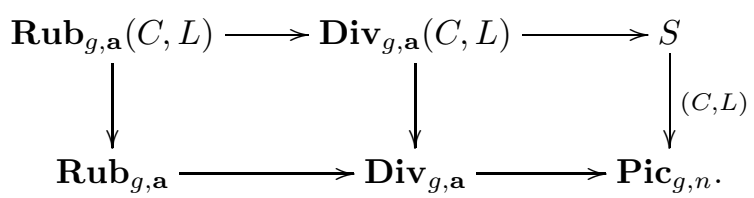

Recall that, by Definition 4.2.1, an $S$-point of $\mathbf{R u b}_{g, \mathbf{a}} \subset \mathbf{D i v}_{g, \mathbf{a}}$ is a logarithmic curve $C$ over $S$, a tropical line bundle $\mathcal{P}$ over $S$, and a map $\alpha: C \rightarrow \mathcal{P}$ over $S$. The factorization through $\mathbf{R u b}_{g, \mathbf{a}}$ induces a division $\mathcal{Q}$ of $\mathcal{P}$, as in Section 5.4, and a logarithmic modification $\tilde{C}=C \times_{\mathcal{P}} \mathcal{Q}$ of $C$.

Let us suppose that $C$ is a stable curve. Locally on $S$, the map $\mathbf{R u b}_{g, \mathbf{a}} \rightarrow \mathbf{P i c}_{g, n}$ gives us a line bundle $L=\mathcal{O}_{C}(\alpha)$ on $C$, well-defined up to tensor product by a line bundle pulled back from $S$. We write $\tilde{L}=\mathcal{O}_{\tilde{C}}(\alpha)$ 
for its pullback to $\tilde{C}$ and $\tilde{L}^{*}=\mathcal{O}_{\tilde{C}}^{*}(\alpha)$ for its associated $\mathbf{G}_{m}$-torsor. Also locally on $S-$ or even globally, by Proposition 5.3.3 - we can choose an isomorphism $\mathcal{P} \simeq \mathbf{G}_{m}^{\text {trop }}$. There is then a $\mathbf{G}_{m}$-torsor $\mathbf{G}_{m}^{\log } \rightarrow \mathbf{G}_{m}^{\text {trop }}$ and, writing $P$ for this torsor, we can identify $L^{*}=C \times_{\mathcal{P}} P$ and $\tilde{L}^{*}=\tilde{C} \times_{\mathcal{P}} P$, by Proposition 4.1.4. We therefore obtain a $\mathbf{G}_{m}$-equivariant map $\tilde{L}^{*} \rightarrow P$. The map $\tilde{C} \rightarrow \mathcal{P}$ factors through $\mathcal{Q}$ by construction of $\tilde{C}$, so the map $\tilde{L}^{*} \rightarrow P$ factors through $Q=\mathcal{Q} \times_{\mathcal{P}} P$. We now have a canonical map $\tilde{L}^{*} \rightarrow Q$ over $S$.

Let $R$ be the space of $\mathbf{G}_{m}$-equivariant maps from $L^{*}$ to $Q$, viewed as a family over $C$, and let $\tilde{R}$ be its pullback to $\tilde{C}$. Perhaps more explicitly, $R=L^{*-1} \otimes Q$. Then $R$ is a family of 2-marked, genus 0 , semistable curves over $C$ and is equipped with a section $\sigma$ over $\tilde{C}$, induced from the $\mathbf{G}_{m}$-equivariant map $\tilde{L}^{*} \rightarrow Q$ constructed above.

Taking stock, we have shown that $(C, \alpha) \in \mathbf{R u b}(S)$ gives rise to the following data:

R1 a semistable logarithmic curve $\tilde{C}$ over $S$ with stabilization $\tau: \tilde{C} \rightarrow C$,

$\mathbf{R 2}$ a family of 2-marked, genus 0 , semistable curves $R$ over $C$,

R3 a divided tropical line $\mathcal{Q}$ over $S$ with $\left[R / \mathbf{G}_{m}\right]=\mathcal{Q} \times{ }_{S} C$,

R4 a section $\sigma$ of $R$ over $\tilde{C}$ such that no component of any fiber of $\tilde{C}$ is carried by $\sigma$ into a node of $\mathcal{Q}$, and

R5 for each component $v$ of $\mathcal{Q}$, the preimage in $\tilde{C}$ contains at least one component that is stable.

The last condition, R5, comes from the construction of $\mathcal{Q}$ and the stability of $C$ : the divisions of $\mathcal{Q}$ occur exactly at the images of components of $C$. We refer to this condition as the stability of $\sigma$.

Proposition 5.5.2. Let $\mathbf{R u b}_{g, \mathbf{a}}^{\mathrm{stab}}$ be the locus of $(C, \alpha)$ in $\mathbf{R u b}_{g, \mathbf{a}}$ such that $C$ is stable. Then $\mathbf{R u b}_{g, \mathbf{a}}^{\mathrm{stab}}$ is equivalent to the stack of quadruples $(\tilde{C}, \mathcal{Q}, R, \sigma)$ as in $\boldsymbol{R} 1-\boldsymbol{R} 5$.

Proof. We have seen already how $\tilde{C}, \mathcal{Q}, R$, and $\sigma$ arise from $(C, \alpha)$ in $\mathbf{R u b}_{g, \mathbf{a}}$. It remains to argue that every datum $(\tilde{C}, \mathcal{Q}, R, \sigma)$ satisfying the conditions arises uniquely by this process.

Let $\tau: \tilde{C} \rightarrow C$ be the stabilization. Since $\mathcal{Q}$ is a divided tropical line, it has a canonical map (in fact, two canonical maps, but we choose the convention in Proposition 5.3.3) to $\mathbf{G}_{m}^{\text {trop }}$. The composition

$$
\tilde{C} \rightarrow R \rightarrow \mathcal{Q} \rightarrow \mathbf{G}_{m}^{\text {trop }}
$$

gives us an object of $\mathbf{R u b}_{g, \mathbf{a}}^{\prime}(\tilde{C})$. We wish to show it descends to $C$.

We take $Q$ to be the pullback of $\mathbf{G}_{m}^{\log }$ to $\mathcal{Q}$ via the map $\mathcal{Q} \rightarrow \mathbf{G}_{m}^{\text {trop }}$ introduced above. Since $\mathcal{R}=\mathcal{Q} \times{ }_{S} C$, Theorem 5.2.5 guarantees that we can recover $R$ as the space of $\mathbf{G}_{m}$-equivariant maps from a $\mathbf{G}_{m}$-torsor $L^{*}$ on $C$ to $Q$. Consider the sheaf $R^{\prime}$ of $\mathbf{G}_{m}$-equivariant maps $R \rightarrow \mathbf{G}_{m}^{\log }$. We will show that the map

$$
\tilde{C} \rightarrow R \rightarrow R^{\prime}
$$

factors through $C$. Since the map (5.5.2.1) factors through $R^{\prime}$ this will give the desired factorization and complete the proof.

We show that

$$
R \rightarrow \tau_{*} \tau^{*} R
$$

is an isomorphism. This is a local assertion in $C$. Near any point $x$ of $C$, we can find an open neighborhood $U$ where $L^{*} \simeq \mathbf{G}_{m}$ and therefore where $R^{\prime} \simeq \mathbf{G}_{m}^{\log }$. That is, sections of $R$ correspond to sections of $M_{C}^{\mathrm{gp}}$ and sections of $\tau^{*} R$ correspond to sections of $M_{\tilde{C}}^{\mathrm{gp}}$. But the map

$$
M_{C}^{\mathrm{gp}} \rightarrow \tau_{*} M_{\tilde{C}}^{\mathrm{gp}}
$$

is an isomorphism, because $\tau$ contracts rational components (see [AMW14, Appendix B] or [CCUW20, Lemma 8.8]). This completes the proof.

The case where an isomorphism between $L$ and $\mathcal{O}_{C}$ is fixed is of particular interest, as this was the situation considered in [GV05]. Here the data simplify to

$\mathbf{R} 1^{\prime}$ a semistable logarithmic curve $\tilde{C}$ with stabilization $\tau: \tilde{C} \rightarrow C$,

$\mathbf{R 2}^{\prime}$ a family of 2-marked, genus 0 , semistable curves $Q$ over $S$,

R3' a morphism $\sigma: \tilde{C} \rightarrow Q$ over $S$ that does not carry any component of $\tilde{C}$ to a node, and

$\mathbf{R} 4^{\prime}$ every component of every fiber of $Q$ is covered by at least one stable component of $\tilde{C}$.

As a result of the above discussion and as special cases of Proposition 5.5.2 we have the following corollaries: 
Corollary 5.5.2.2. Let $\operatorname{Rub}\left(\mathcal{M}_{g, n}^{\log }, \mathcal{O}\right)$ be the substack of $\mathbf{R u b}$ parameterizing those $(C, \alpha)$ where $C$ is stable and $\mathcal{O}_{C}(\alpha)$ is pulled back from the base. Then $\operatorname{Rub}\left(\mathcal{M}_{g, n}^{\log }, \mathcal{O}\right)$ is isomorphic to the moduli space of data $R 1^{\prime}-R 4^{\prime}$.

Corollary 5.5.2.3. $\operatorname{Rub}\left(\mathcal{M}_{g, n}^{\log }, \mathcal{O}\right)$ is isomorphic the moduli space of rubber stable maps.

5.6. The rubber virtual fundamental class. Graber and Vakil gave a pointwise first-order deformation space and a 2-step obstruction space for $\mathbf{R u b}_{g, \mathbf{a}}\left(\mathcal{M}_{g, n}^{\log }, \mathcal{O}\right)$ over $\mathfrak{M}_{g, n}^{\log } \times \mathfrak{M}_{0,2}^{\mathrm{ss}}[\mathrm{GV05}] .^{4}$ In this section, we will describe several global complexes that control the deformation and obstructions of $\mathbf{R u b}_{g, \mathbf{a}}\left(\mathcal{M}_{g, n}^{\log }, \omega^{\otimes k}\right)$ relative to different bases. We relate our obstruction complexes to Graber and Vakil's deformation and obstruction spaces in Section 5.6.6.

Theorem 5.6.1. The obstruction theories for $\mathbf{R u b}_{g, \mathbf{a}}\left(\mathcal{M}_{g, n}^{\log }, \omega^{k}\right)$ described in Sections 4.6, 5.6.2, and 5.6.4 all give the same virtual fundamental class.

The proof of this theorem is given in Sections 5.6.2 through 5.6.4 below.

5.6.2. A second obstruction theory. Recall that $\mathbf{R u b}_{g, \mathbf{a}}\left(\mathcal{M}_{g, n}^{\log }, \omega^{\otimes k}\right)$ is constructed from a cartesian diagram (5.6.2.1) where the map $\mathcal{M}_{g, n}^{\log } \rightarrow \mathbf{P i c}_{g, n}$ sends a curve $C$ to $\omega_{C}^{\otimes k}$ :

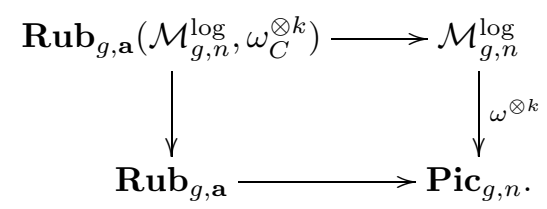

Section 4.6 gives a virtual fundamental class of $\mathbf{R u b}_{g, \mathbf{a}}\left(\mathcal{M}_{g, n}^{\log }, \omega^{\otimes k}\right)$ by way of the canonical relative logarithmic obstruction theory associated to the morphism of logarithmically smooth algebraic stacks aj : $\mathbf{R u b}_{g, \mathbf{a}} \rightarrow$ $\mathbf{P i c}_{g, n}$.

However, the map $\mathcal{M}_{g, n}^{\log } \rightarrow \mathbf{P i c}_{g, n}$ is a locally closed embedding of smooth stacks, with normal bundle $H^{1}\left(C, \mathcal{O}_{C}\right)$ at a curve $C$. Therefore there is a relative obstruction theory for $\mathbf{R u b}_{g, \mathbf{a}}\left(\mathcal{M}_{g, n}^{\log }, \omega^{k}\right)$ over $\mathbf{R u b}_{g, \mathbf{a}}$ with obstruction bundle $H^{1}\left(C, \mathcal{O}_{C}\right)$ giving another virtual fundamental class on $\operatorname{Rub}_{g, \mathbf{a}}\left(\mathcal{M}_{g, n}^{\log }, \omega^{k}\right)$ by Gysin pullback from $\mathbf{R u b}_{g, \mathbf{a}}$.

We claim that these two virtual fundamental classes coincide. In order to see this, we must recall that if $f: X \rightarrow Y$ is a morphism of logarithmic algebraic stacks with a perfect relative logarithmic obstruction theory $E$, then $E$ gives an obstruction theory for the map $g: \underline{X} \rightarrow \log (Y)$ induced from $f$, where $\underline{X}$ is the underlying algebraic stack of $X$. Indeed, the relative logarithmic cotangent complex of $X \rightarrow Y$ is the relative cotangent complex of $\underline{X} \rightarrow \log (Y)$, by definition [Ols05, Definition 3.2]. If $Y$ is logarithmically regular then $\underline{Y}$ is dense in $\log (Y)$ (one can see this from the local charts [Ols03, Corollary 5.25]) so $\log (Y)$ is equidimensional if $Y$ is, of the same dimension as $Y$. In this case, the relative logarithmic virtual fundamental class is defined to be $g^{!}[\log (Y)]$.

Since $\mathcal{M}_{g, n}^{\log } \rightarrow \mathbf{P i c}_{g, n}$ is strict, so is $\mathbf{R u b}_{g, \mathbf{a}}\left(\mathcal{M}_{g, n}^{\log }, \omega_{C}^{\otimes k}\right) \rightarrow \mathbf{R u b}_{g, \mathbf{a}}$. We can therefore turn diagram (5.6.2.1) into a cartesian diagram of algebraic stacks (5.6.2.2), ignoring logarithmic structures:

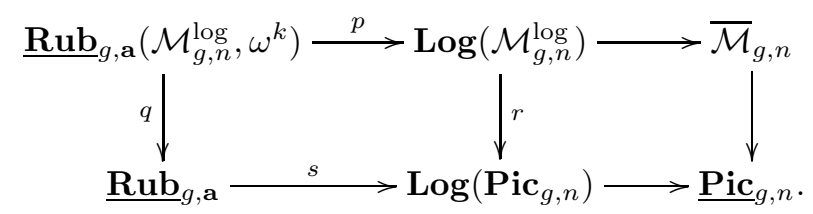

The underlines denote underlying algebraic stacks (with logarithmic structure forgotten). The square on the right is cartesian by the strictness of $\overline{\mathcal{M}}_{g, n} \rightarrow \underline{\mathbf{P i c}}_{g, n}$ and the outer square is cartesian because $\overline{\mathcal{M}}_{g, n} \rightarrow \underline{\mathbf{P i c}}_{g, n}$ is strict and (5.6.2.1) is cartesian.

By [Man12, Theorem 4.3], we have

$$
q^{!}\left[\underline{\mathbf{R u b}}_{g, \mathbf{a}}\right]=q^{!} s^{!}\left[\mathbf{L o g}\left(\mathbf{P i c}_{g, n}\right)\right]=p^{!} r^{!}\left[\mathbf{L o g}\left(\mathbf{P i c}_{g, n}\right)\right]=p^{!}\left[\mathbf{L o g}\left(\overline{\mathcal{M}}_{g, n}\right)\right]
$$

so the two virtual fundamental classes coincide.

\footnotetext{
${ }^{4}$ Op. cit. asserts that this is an obstruction theory relative to $\mathfrak{M}_{g, n}^{\log } \times \mathscr{T}$, but this appears to have been a misstatement.
} 
5.6.3. The source of the obstruction. In Section 5.6.4, we will describe still another obstruction theory for $\operatorname{Rub}_{g, n}\left(\mathcal{M}_{g, n}^{\log }, \omega^{k}\right)$. In order to compare that one to the obstruction theories just introduced, we will need to understand how obstructions in $H^{1}\left(C, \mathcal{O}_{C}\right)$ arise in practice in the obstruction theory introduced in Section 5.6.2. Consider a square-zero logarithmic lifting problem (5.6.3.1) where $S^{\prime}$ is a strict square-zero extension of $S$ with ideal $J$ :

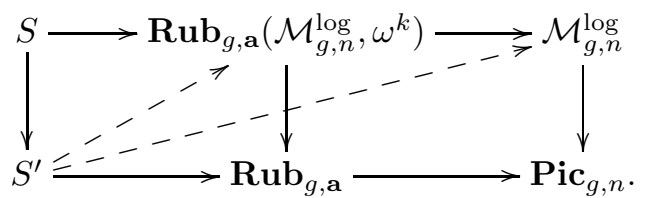

It is equivalent to produce either of the dashed arrows, since the square on the right is cartesian. The map $S \rightarrow \mathcal{M}_{g, n}^{\log }$ gives a logarithmic curve $C$ over $S$, and the map $S^{\prime} \rightarrow \mathbf{P i c}_{g, n}$ gives a logarithmic curve $C^{\prime}$ over $S^{\prime}$ extending $C$ and an equivalence class of line bundles $L^{\prime}$ on $C^{\prime}$ (equivalence being tensor product by line bundles pulled back from $S^{\prime}$ ) whose restriction to $C$ is pulled back from $S$. If we pick an isomorphism $\psi: \omega_{C / S}^{k} \simeq L$, then the lifts of $\psi$ to an isomorphism $\psi^{\prime}: \omega_{C^{\prime} / S^{\prime}}^{k} \simeq L^{\prime}$ form a torsor under

$$
\operatorname{Hom}\left(\omega_{C^{\prime} / S^{\prime}}^{k}, J L^{\prime}\right)=\operatorname{Hom}\left(\omega_{C / S}^{k}, J \otimes \omega_{C / S}^{k}\right)=J
$$

so there is an obstruction in $H^{1}(C, J)=J \otimes H^{1}\left(C, \mathcal{O}_{C}\right)$.

More specifically, the map $S^{\prime} \rightarrow \mathbf{R u b}_{g, \mathbf{a}}$ gives a family of divided tropical lines $\mathcal{Q}^{\prime}$ over $S^{\prime}$ and a map $\alpha: C^{\prime} \rightarrow \mathcal{Q}^{\prime}$. The line bundle $L^{\prime}$ above, obstructing the lift, is $\mathcal{O}_{C^{\prime}}(\alpha)$.

We note, however, that the isomorphism $\psi$ may only exist locally on $S$. It is therefore more canonical to choose a line bundle $K$ on $S$ and an isomorphism $\phi: \pi^{*} K \otimes \omega_{C / S}^{k} \rightarrow L$ on $C$. The pair $(K, \phi)$ is uniquely determined up to unique isomorphism by $L$ (indeed, $\left.K=\pi_{*} \operatorname{Hom}\left(\omega_{C / S}^{k}, L\right)\right)$. The choices of lifts of $K$ to a line bundle $K^{\prime}$ on $S^{\prime}$ are controlled by the complex $\mathcal{O}_{S}[1]$. Once such a lift has been chosen, the lifts of the isomorphism $\phi$ to $\phi^{\prime}: \pi^{*} K^{\prime} \otimes \omega_{C^{\prime} / S^{\prime}}^{k} \rightarrow L^{\prime}$ form a torsor under $\operatorname{Hom}\left(\pi^{*} K \otimes \omega_{C / S}^{k}, L \otimes J\right)=J$, by the calculation in the last paragraph, so that we have a map

$$
\mathcal{O}_{S}[1] \rightarrow \mathrm{R} \pi_{*} \mathcal{O}_{C}[1]
$$

whose cone, $\mathrm{R}^{1} \pi_{*} \mathcal{O}_{C}$, is the obstruction bundle of a relative obstruction theory for $\mathbf{R u b}_{g, \mathbf{a}}\left(\mathcal{M}_{g, n}^{\log }, \omega^{k}\right)$ over $\mathbf{R u b}_{g, \mathbf{a}}$. This proves the following proposition:

Proposition 5.6.3.2. There is a relative obstruction theory for $\mathbf{R u b}_{g, \mathbf{a}}\left(\mathcal{M}_{g, n}^{\log }, \omega^{k}\right)$ over $\mathbf{R u b}_{g, \mathbf{a}}$ with obstruction bundle $\mathrm{R}^{1} \pi_{*} \mathcal{O}_{C}$.

5.6.4. A third obstruction theory. As explained in Section $5.5, \mathbf{R u b}_{g, \mathbf{a}}\left(\mathcal{M}_{g, n}^{\log }, \omega^{k}\right)$ is the space of a logarithmic curve $C$, a family of 2-marked, semistable, rational curves $Q$, and a stable (logarithmic) section of the twisted family $\omega_{C}^{-k} \otimes Q$ over $C$. By forgetting the section, there is a logarithmic map

$$
\operatorname{Rub}_{g, \mathbf{a}}\left(\mathcal{M}_{g, n}^{\log }, \omega^{k}\right) \rightarrow \mathfrak{M}_{g, n}^{\log } \times \mathfrak{M}_{0,2}^{\mathrm{ss}} .
$$

In the case $k=0$, Graber and Vakil describe a 2-step schematic - that is, not logarithmic - obstruction theory for this map. We will also give a 2-step obstruction theory, by thinking of the schematic morphism as a logarithmic morphism (this section) followed by a change of logarithmic structure (Section 5.6.5). The filtered pieces of this obstruction theory are not the same as Graber and Vakil's, although we will see that both theirs and ours have a common refinement. Ours has the advantage that both steps of the filtration are perfect obstruction theories.

Proposition 5.6.4.2. There is a relative logarithmic obstruction theory for $\mathbf{R u b}_{g, \mathbf{a}}\left(\mathcal{M}_{g, n}^{\log }, \omega^{k}\right)$ over $\mathfrak{M}_{g, n}^{\log } \times$ $\mathfrak{M}_{0,2}^{\mathrm{ss}}$ with obstruction complex $\mathrm{R} \pi_{*} f^{*} T_{\left(\omega_{C / S}^{-k} \otimes Q\right) / C}[1]$.

In fact, the following lemma shows that $T_{\left(\omega_{C / S}^{-k} \otimes Q\right) / C}$ is canonically isomorphic to $\mathcal{O}_{C}$. We've used the more complicated notation to emphasize the geometric origin of the obstructions.

Lemma 5.6.4.3. Let $Q$ be a family of 2-marked, semistable, rational curves over $S$. Then the relative logarithmic tangent bundle $T_{Q / S}$ is canonically trivial. 
Proof. One way to see this is to note that $Q$ is a division of a logarithmic line bundle $P$. Since $P$ is a torsor under $\mathbf{G}_{m}^{\log }$, its tangent bundle is the lie algebra of $\mathbf{G}_{m}^{\log }$ (which is isomorphic to $\mathcal{O}_{S}$ ) twisted by $P$, via the adjoint action. But $\mathbf{G}_{m}^{\log }$ is commutative, so the adjoint action is trivial, and $T_{Q / S}=T_{\mathbf{G}_{m}^{\log }}=\mathcal{O}_{Q}$.

A more direct argument is to note that $Q$ is locally isomorphic to $\mathbf{A}_{S}^{1}$ or to the pullback of the multiplication map $\mathbf{A}^{2} \rightarrow \mathbf{A}^{1}$ along a logarithmic morphism $S \rightarrow \mathbf{A}^{1}$. In either case, these isomorphisms are uniquely determined up to scaling by elements of $\mathcal{O}_{S}^{*}$. By we have canonical trivializations of the duals of $T_{\mathbf{A}^{1}}$ and $T_{\mathbf{A}^{2} / \mathbf{A}^{1}}$ given by $\frac{d x}{x}$. These trivializations are independent of scaling by $\mathcal{O}_{S}^{*}$ and can be seen easily to glue to a global trivialization of $T_{Q / S}$.

The construction of the obstruction theory in Proposition 5.6.4.2 is standard, but in order to compare this obstruction theory to the one from Sections 5.6.2 and 5.6.3, it will be necessary to analyze exactly how an obstruction arises from a deformation problem (5.6.4.4):

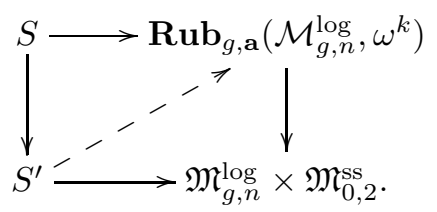

The map $S \rightarrow \mathbf{R u b}_{g, \mathbf{a}}\left(\mathcal{M}_{g, n}^{\log }, \omega^{k}\right)$ gives a semistable logarithmic curve $C$ over $S$, a semistable family of 2-marked, rational logarithmic curves $Q$ over $S$, and a section $f$ of $\omega_{C / S}^{-k} \otimes Q$ over $C$. We write $\mathcal{Q}=\left[Q / \mathbf{G}_{m}\right]$ and $\alpha$ for the composition $C \rightarrow \omega_{C / S}^{-k} \otimes Q \rightarrow \pi^{-1} \mathcal{Q}$.

The map $S^{\prime} \rightarrow \mathfrak{M}_{g, n}^{\log } \times \mathfrak{M}_{0,2}^{\text {ss }}$ gives a semistable logarithmic curve $C^{\prime}$ over $S^{\prime}$ extending $C$ and a family of 2-marked, semistable, rational curves $Q^{\prime}$ over $S^{\prime}$ extending $Q$. Since $Q^{\prime}$ is logarithmically smooth, there is no local obstruction to finding an arrow $C^{\prime} \rightarrow \omega_{C^{\prime} / S^{\prime}}^{-k} \otimes Q^{\prime}$ in diagram (5.6.4.5):

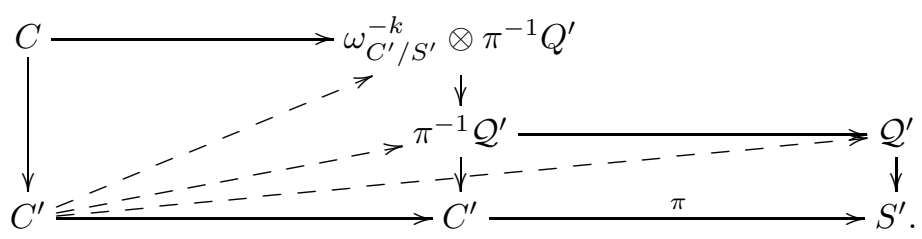

Choices of lift form a torsor under $f^{*} T_{\left(\omega_{C / S}^{-k} \otimes Q\right) / C}$, and the class of this torsor in $\mathrm{R}^{1} \pi_{*} f^{*} T_{\left(\omega_{C / S}^{-k} \otimes Q\right.}$ serves as the obstruction. This proves Proposition 5.6.4.2, but we analyze the obstruction a bit further to show it agrees with the obstruction from Section 5.6.3.

We note now that because $\omega_{C / S}^{-k} \otimes Q$ is a family of 2-marked, rational, semistable curves over $S$, its logarithmic tangent bundle $T_{\left(\omega_{C / S}^{-k} \otimes Q\right) / C}$ is canonically trivial by Lemma 5.6.4.3. Therefore the obstruction complex here is canonically isomorphic to $\mathrm{R} \pi_{*} \mathcal{O}_{C}$. In order to compare this obstruction theory to the one from Sections 5.6.2 and 5.6.3, we will need to show that the map

$$
\mathrm{R} \pi_{*} f^{*} T_{\omega_{C / S}^{-k} \otimes Q / C}[1] \simeq \mathrm{R} \pi_{*} \mathcal{O}_{C}[1] \rightarrow \mathrm{R}^{1} \pi_{*} \mathcal{O}_{C}
$$

carries the obstruction constructed here to the one constructed in Section 5.6.3.

Recall that if $\alpha^{\prime}$ is the piecewise linear function on the dual graph of $C^{\prime}$ classified by $S^{\prime} \rightarrow \mathbf{R u b}_{g, \mathbf{a}}$, and $\alpha$ is its restriction to $C$, then the obstruction constructed in Section 5.6.3 is the torsor of trivializations of $\omega_{C^{\prime} / S^{\prime}}^{-k}\left(\alpha^{\prime}\right)$ lifting the trivialization of $\omega_{C / S}^{-k}(\alpha)$ specified by $S \rightarrow \mathbf{R u b}_{g, \mathbf{a}}\left(\mathcal{M}_{g, n}^{\log }, \omega^{k}\right)$.

We write $\mathcal{Q}=\left[Q / \mathbf{G}_{m}\right]$ and $\mathcal{Q}^{\prime}=\left[Q^{\prime} / \mathbf{G}_{m}\right]$. By definition, the obstruction constructed in this section is the torsor of lifts $C^{\prime} \rightarrow \omega_{C^{\prime} / S^{\prime}}^{-k} \otimes Q^{\prime}$ of (5.6.4.5) extending the given map over $C$. However, $\mathcal{Q}^{\prime}$ is logarithmically étale over $S^{\prime}$, so there is a unique extension $C^{\prime} \rightarrow \mathcal{Q}^{\prime}$ of $C \rightarrow \mathcal{Q}$ as in Diagram (5.6.4.5). Therefore lifting (5.6.4.5) is equivalent to lifting

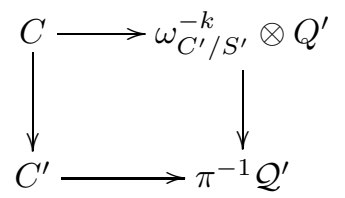


Now, $Q^{\prime}$ is a $\mathbf{G}_{m}$-torsor over $\mathcal{Q}^{\prime}$. In fact, by Proposition 4.1.4, we know that $Q^{\prime}=\mathcal{O}_{C^{\prime}}^{*}\left(\alpha^{\prime}\right)$. Therefore the torsor of lifts of (5.6.4.6) is the same as the torsor of trivializations of $\omega_{C^{\prime} / S^{\prime}}^{-k}\left(\alpha^{\prime}\right)$, which (as we noted above) is the obstruction constructed in Section 5.6.3.

This completes the proof of Theorem 5.6.1.

5.6.5. Deforming the logarithmic structure. Graber and Vakil do not work in the logarithmic category, and the map (5.6.4.1) is not strict. Therefore the first obstruction to lifting a diagram (5.6.4.4) of schemes is to promote it to a diagram in the category of logarithmic schemes, in which $S \rightarrow S^{\prime}$ is strict and $S$ has the logarithmic structure pulled back from $\mathbf{R u b}_{g, \mathbf{a}}$.

Remark 5.6.5.1. We will see that the space of promotions alluded to above is a birational modification of the base. Since we know that the fundamental class should pull back to the fundamental class under a birational modification, we don't actually need an obstruction theory for this map. The theory of logarithmic obstruction theories and virtual fundamental classes formalizes this.

Remark 5.6.5.2. It is possible to combine the obstruction theory presented in this section with the logarithmic obstruction theory in Section 5.6.4 and recognize them as filtered pieces of a 1-step obstruction theory. However, it seems difficult to describe the obstruction in a natural way, owing to the very different sources of the two types of obstructions (one being local to $S$ and the other being local to $C$ ). Li dealt with a related issue in his moduli space of stable maps to expanded targets [Li01, Li02] (see also [CMW12]).

One advantage of working with logarithmic obstruction theories is that they frequently allow us to bypass such issues.

To emphasize that this problem is purely about deforming logarithmic strctures, consider the factorization (5.6.5.3) of (5.6.4.1):

$$
\operatorname{Rub}_{g, \mathbf{a}}\left(\mathcal{M}_{g, n}^{\log }, \omega^{k}\right) \rightarrow \mathbf{R u b}_{g, n} \times \mathbf{B G}_{m} \rightarrow \mathfrak{M}_{g, n}^{\log } \times \mathscr{T} \times \mathbf{B G}_{m} \simeq \mathfrak{M}_{g, n}^{\log } \times \mathfrak{M}_{0,2}^{\mathrm{ss}}
$$

The first map sends an $\alpha: C \rightarrow \mathcal{Q}$ such that $\mathcal{O}_{C}(\alpha)$ differs from $\omega_{C}^{k}$ by a line bundle pulled back from the base to $\alpha: C \rightarrow \mathcal{Q}$ and the line bundle that pulls back to $\omega_{C}^{-k}(\alpha)$. The second map is logarithmically étale, so our logarithmic obstruction theories over $\mathfrak{M}_{g, n}^{\log } \times \mathfrak{M}_{0,2}^{\mathrm{ss}}$ are also obstruction theories over $\mathbf{R u b} \mathbf{b}_{g, n} \times \mathbf{B G}_{m}$. We will describe a schematic obstruction theory for the second map.

Consider a schematic lifting problem (5.6.5.4), in which $S^{\prime}$ is a square-zero extension of $S$ :

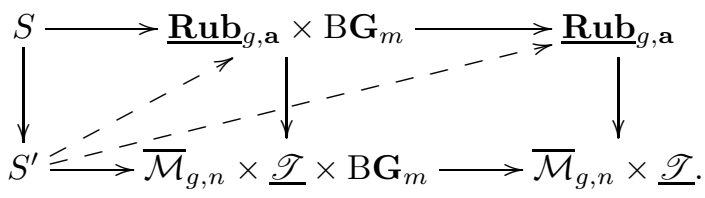

As usual, underlines denote underlying algebraic stacks. It is equivalent to produce either dashed arrow, so we focus on the outer rectangle.

We write $M_{S}$ for the logarithmic structure of $S$ pulled back from $\mathbf{R u b} \mathbf{b}_{g, \mathbf{a}}$, and $M_{S^{\prime}}$ for the logarithmic structure of $S^{\prime}$ pulled back from $\mathcal{M}_{g, n}^{\log } \times \mathscr{T}$. If the dashed arrow exists, it gives a second logarithmic structure $M_{S^{\prime}}^{\prime}$ on $S^{\prime}$ and a factorization

$$
\left(S, M_{S}\right) \rightarrow\left(S^{\prime}, M_{S^{\prime}}^{\prime}\right) \rightarrow\left(S^{\prime}, M_{S^{\prime}}\right)
$$

in which the first arrow is strict. Conversely, any such factorization gives a lift of (5.6.5.4) because $\mathbf{R u b}_{g, \mathbf{a}} \rightarrow$ $\mathfrak{M}_{g, n}^{\log } \times \mathscr{T}$ is logarithmically étale. Thus, lifting (5.6.5.4) is equivalent to finding a factorization (5.6.5.5).

Working locally in $S$, we describe an obstruction complex controlling these lifts. We may assume, without loss of generality, that $S$ has a geometric point such that the maps

$$
\begin{aligned}
\Gamma\left(S, \bar{M}_{S}\right) & \rightarrow \bar{M}_{S, s} \\
\Gamma\left(S^{\prime}, \bar{M}_{S^{\prime}}\right) & \rightarrow \bar{M}_{S^{\prime}, s}
\end{aligned}
$$

are both bijections.

Let $\mathfrak{f}_{s}: \mathfrak{C}_{s} \rightarrow \mathfrak{Q}_{s}$ be the tropicalization of the map $f_{s}: C_{s} \rightarrow Q_{s}$ (see [CCUW20, Section 7]). The structure of the monoids $\bar{M}_{S, s}$ and $\bar{M}_{S^{\prime}, s}$ can be read off from $\mathfrak{f}_{s}$. The monoid $\bar{M}_{S, s}$ is freely generated by the lengths $\delta(e)$ as $e$ ranges among the edges of $\mathfrak{C}_{s}$ and $\mathfrak{Q}_{s}$. The monoid $\bar{M}_{S^{\prime}, s}$ is the quotient of $\bar{M}_{S, s}$ in 
which $k \delta(e)$ is identified with $\delta\left(e^{\prime}\right)$ if $e$ is an edge of $\mathfrak{C}_{s}$ that maps to an edge $e^{\prime}$ of $\mathfrak{Q}_{s}$ with expansion factor $k$.

The monoid of relations on $\bar{M}_{S^{\prime}, s}$ defining $\bar{M}_{S, s}$ is freely generated by the relations $k \delta(e) \sim \delta\left(e^{\prime}\right)$, described above. To build $M_{S^{\prime}}^{\prime}$, we must therefore choose an identification $\mathcal{O}_{S^{\prime}}(k \delta(e)) \simeq \mathcal{O}_{S^{\prime}}\left(\delta\left(e^{\prime}\right)\right)$ for each of these relations, extending the identity $\mathcal{O}_{S}(k \delta(e))=\mathcal{O}_{S}\left(\delta\left(e^{\prime}\right)\right)$. The choices for each of these identifications form a torsor under $\mathcal{O}_{S}$.

Once isomorphisms $\varphi_{e}: \mathcal{O}_{S^{\prime}}(k \delta(e)) \rightarrow \mathcal{O}_{S^{\prime}}\left(e^{\prime}\right)$ have been chosen, we have an obstruction, coming from the need for the maps

$$
\begin{aligned}
\varepsilon_{k \delta(e)}: \mathcal{O}_{S^{\prime}} \rightarrow \mathcal{O}_{S^{\prime}}(k \delta(e)) \\
\varepsilon_{\delta\left(e^{\prime}\right)}: \mathcal{O}_{S^{\prime}} \rightarrow \mathcal{O}_{S^{\prime}}\left(\delta\left(e^{\prime}\right)\right)
\end{aligned}
$$

to agree, namely for the difference $\varepsilon_{\delta\left(e^{\prime}\right)}-\varphi_{e} \varepsilon_{k \delta(e)}$ to vanish. Since this difference already vanishes over $S$, it can be regarded as a map

$$
\mathcal{O}_{S} \rightarrow \mathcal{O}_{S}\left(\delta\left(e^{\prime}\right)\right)
$$

We therefore obtain a tangent-obstruction complex. This is summarized by the following proposition.

Proposition 5.6.5.6. The map $\mathbf{R u b}_{g, n} \rightarrow \mathcal{M}_{g, n}^{\log } \times \mathscr{T}$ has a schematic relative tangent-obstruction complex

$$
\prod_{e \rightarrow e^{\prime}} \mathcal{O}_{S} \rightarrow \prod_{e \rightarrow e^{\prime}} \mathcal{O}_{S}\left(\delta\left(e^{\prime}\right)\right)
$$

where the products are taken over edges of $\mathfrak{C}_{s}$ mapping to edges of $\mathfrak{Q}_{s}$. The differential sends $\varphi_{e}$ in the $e \rightarrow e^{\prime}$ factor to $\varepsilon_{\delta\left(e^{\prime}\right)}-\varphi_{e} \varepsilon_{k \delta(e)}$ in the same factor of the target.

Remark 5.6.5.7. In fact, $\mathbf{R u b}_{g, n} \rightarrow \mathcal{M}_{g, n}^{\log } \times \mathscr{T}$ is a local complete intersection morphism and (5.6.5.6.1) is its relative tangent complex. In the Behrend-Fantechi formalism for obstruction theories, the obstruction theory is encoded by an isomorphism from the dual of (5.6.5.6.1) to the relative cotangent complex.

5.6.6. Comparison to Graber and Vakil's obstruction theory. For simplicity, we will work on $\mathbf{R u b}_{g, n}\left(\mathcal{M}_{g, n}^{\log }, \mathcal{O}\right)$. By Proposition 5.5.2, we can identify $\operatorname{Rub}_{g, n}\left(\mathcal{M}_{g, n}^{\log }, \mathcal{O}\right)$ with the space of stable (logarithmic) maps from logarithmic curves $C$ to 2 -marked, semistable, rational curves $Q$.

Given such a map $f: C \rightarrow Q$, Graber and Vakil define a sheaf $f^{\dagger} T_{Q}(\log D)$ as the quotient of $f^{*} T_{\underline{Q}}(\log D)$ by its torsion subsheaf (where $D$ is the divisor at 0 and $\infty$ of $Q$ and $T_{\underline{Q}}$ is the tangent sheaf of the underlying scheme of $Q$ ).

Proposition 5.6.6.1. There is an exact sequence

$$
0 \rightarrow f^{*} T_{Q} \rightarrow f^{\dagger} T_{Q} \rightarrow \prod_{e \rightarrow e^{\prime}} \mathcal{O}_{S} \rightarrow 0
$$

where the product is taken over the nodes e of $C$ that are carried by $f$ to nodes of $Q$.

Proof. Away from the nodes of $C$ that map to nodes of $Q$, the map $f^{*} T_{Q} \rightarrow f^{\dagger} T_{Q}$ is an isomorphism, so we work in a neighborhood of such a node $e$ with image $e^{\prime}$. Let $x$ and $y$ be parameters for the branches of $Q$ at $e^{\prime}$ and let $v$ and $w$ be coordinates of $C$ at the branches of $e$. After exchanging $v$ and $w$ if necessary, $x$ pulls back to $v^{k}$ and $y$ pulls back to $w^{k}$.

We can identify $T_{\underline{Q}}$ as the set of derivations from $\mathcal{O}_{\underline{Q}}$ into itself. It is generated by the tangent vector fields $\delta_{x}$ and $\delta_{y}$ where

$$
\begin{array}{ll}
\delta_{x}(d x)=x & \delta_{y}(d x)=0 \\
\delta_{x}(d y)=0 & \delta_{y}(d y)=y
\end{array}
$$

with the relations $y \delta_{x}=x \delta_{y}=0$. Then $f^{\dagger} T_{Q}$ is generated by $\delta_{x}$ and $\delta_{y}$ over $\mathcal{O}_{C}$, with the relations $w \delta_{x}=v \delta_{y}=0$.

Now, $T_{Q}$ is freely generated by $\delta_{\log x}$, where $\delta_{\log x}(d \log x)=1$ (and $\delta_{\log x}(d \log y)=-1$ ). We note that $\delta_{\log x}(d x)=x$ and $\delta_{\log x}(d y)=-y$ so the map $T_{Q} \rightarrow T_{Q}$ sends $\delta_{\log x}$ to $\delta_{x}-\delta_{y}$.

As $v \delta_{x}=v\left(\delta_{x}-\delta_{y}\right)$ and $w \delta_{y}=w\left(\delta_{x}-\delta_{y}\right)$ in $f^{\dagger} T_{Q}$, it follows that $f^{\dagger} T_{Q} / f^{*} T_{Q}$ is a skyscraper sheaf of length 1 supported at $e$ and spanned by $\delta_{x} \equiv \delta_{y}$. On the other hand, $x$ is well-defined up to multiplication by a unit, and we argue that $\delta_{x} \bmod f^{*} T_{Q}$ is independent of this ambiguity. 
Indeed, suppose that $u x$ is another local parameter for the same component as is $x$ (so $u$ is an invertible function). We have $\delta_{u x}(d x)=x-u^{-1} x \delta_{u x}(d u)$, so

$$
\begin{aligned}
& \left(\delta_{u x}-\delta_{x}\right)(d x)=-u^{-1} x \delta_{u x}(d u) \\
& \left(\delta_{u x}-\delta_{x}\right)(d y)=0 .
\end{aligned}
$$

There is a unique logarithmic tangent vector field $\gamma$ such that $\gamma(d \log x)=-u^{-1} \delta_{u x}(d u)$. Then

$$
\begin{aligned}
& \gamma(d x)=x \gamma(d \log x)=-u^{-1} x \delta_{u x}(d u) \\
& \gamma(d y)=-y \gamma(d \log x)=u^{-1} y \delta_{u x}(d u)=0,
\end{aligned}
$$

with the last equality because $\delta_{u x}(d u)$ is a multiple of $x$. Therefore $\delta_{u x}-\delta_{x}$ is in $f^{*} T_{Q}$ and the image of $\delta_{x}$ in $f^{\dagger} T_{\underline{Q}}$ is a canonical generator.

We can now prove Theorem C. In [GV05, p. 10, Equation (3)], Graber and Vakil find a primary obstruction to deforming $C \rightarrow Q$ in

$$
H^{0}\left(C, f^{-1} \operatorname{Ext}^{1}\left(\Omega_{\underline{Q}}(\log D), \mathcal{O}_{Q}\right)\right)=\prod_{e \rightarrow e^{\prime}} \mathcal{O}_{S}\left(\delta\left(e^{\prime}\right)\right),
$$

which is the product of the deformation spaces of the nodes $e^{\prime}$ of $Q$, and therefore coincides with $\prod_{e \rightarrow e^{\prime}} \mathcal{O}_{S}\left(\delta\left(e^{\prime}\right)\right)$, the second term of the obstruction complex in Proposition 5.6.5.6. There is a secondary obstruction ${ }^{5}$ in

$$
H^{1}\left(C, f^{\dagger} T_{Q}\right)
$$

which is the image of the obstruction space $H^{1}\left(C, f^{*} T_{Q}\right)=H^{1}\left(C, \mathcal{O}_{C}\right)$ from Proposition 5.6.4.2. When these obstructions vanish, the deformations are a torsor under

$$
H^{0}\left(C, f^{\dagger} T_{Q}\right)
$$

Therefore the class in K-theory of Graber and Vakil's obstruction theory is the following virtual bundle:

$$
\prod_{e \rightarrow e^{\prime}} \mathcal{O}_{S}\left(\delta\left(e^{\prime}\right)\right)-\mathrm{R} \pi_{*} f^{\dagger} T_{Q}
$$

On the other hand, the factorization

$$
\underline{\mathbf{R u b}}_{g, n}\left(\mathcal{M}_{g, n}^{\log }, \mathcal{O}\right) \rightarrow \underline{\mathbf{R u b}}_{g, n} \rightarrow \mathfrak{M}_{g, n}^{\log } \times \mathscr{T}
$$

gives another 2-step obstruction theory. By Propositions 5.6.4.2, 5.6.5.6, and 5.6.6.1 the $K$-theory class of this obstruction theory is the following sum:

$$
\begin{aligned}
& \prod_{e \rightarrow e^{\prime}} \mathcal{O}_{S}\left(\delta\left(e^{\prime}\right)\right)-\prod_{e \rightarrow e^{\prime}} \mathcal{O}_{S}-\mathrm{R} \pi_{*} f^{*} T_{Q} \\
= & \prod_{e \rightarrow e^{\prime}} \mathcal{O}_{S}\left(\delta\left(e^{\prime}\right)\right)-\prod_{e \rightarrow e^{\prime}} \mathcal{O}_{S}-\mathrm{R} \pi_{*} f^{\dagger} T_{Q}+\prod_{e \rightarrow e^{\prime}} \mathcal{O}_{S} \\
= & \prod_{e \rightarrow e^{\prime}} \mathcal{O}_{S}\left(\delta\left(e^{\prime}\right)\right)-\mathrm{R} \pi_{*} f^{\dagger} T_{Q}
\end{aligned}
$$

This completes our comparison to Graber and Vakil's construction, and the proof of Theorem C.

Remark 5.6.6.2. One could consider a scheme $S$ equipped with a family of nodal curves $C$ and an invertible sheaf $L$ on $C$ such that the associated map $S \rightarrow \mathbf{P i c}_{g, n}$ has a perfect relative obstruction theory. This gives a perfect relative obstruction theory for the $\operatorname{map} \underline{\mathbf{R u b}}_{g, n}(C, L) \rightarrow \underline{\mathbf{R u b}}_{g, n}$. Combined with Proposition 5.6.5.6, this gives a 2-step obstruction theory for the $\operatorname{map} \underline{\mathbf{R u b}}_{g, n}(C, L) \rightarrow \mathfrak{M}_{g, n}^{\log } \times \mathscr{T}$.

\footnotetext{
${ }^{5}$ There is a typographical error in Equation (2) of loc. cit.: the secondary obstruction group is $H^{1}\left(C, f^{\dagger} T_{X_{l}}\left(-\log D_{\infty}\right)\right)$, as in Equation (3), and not $H^{1}\left(C, f^{*} T_{X_{l}}\left(-\log D_{\infty}\right)\right)$.
} 


\section{REFERENCES}

[ACFW13] Dan Abramovich, Charles Cadman, Barbara Fantechi, and Jonathan Wise, Expanded degenerations and pairs, Comm. Algebra 41 (2013), no. 6, 2346-2386. MR 3225278

[ACG $\left.{ }^{+} 13\right]$ Dan Abramovich, Qile Chen, Danny Gillam, Yuhao Huang, Martin Olsson, Matthew Satriano, and Shenghao Sun, Logarithmic geometry and moduli, Handbook of moduli. Vol. I, Adv. Lect. Math. (ALM), vol. 24, Int. Press, Somerville, MA, 2013, pp. 1-61. MR 3184161

$\left[\mathrm{ACM}^{+} 16\right]$ Dan Abramovich, Qile Chen, Steffen Marcus, Martin Ulirsch, and Jonathan Wise, Skeletons and fans of logarithmic structures, Nonarchimedean and tropical geometry, Simons Symp., Springer, [Cham], 2016, pp. 287-336. MR 3702314

[AMW14] Dan Abramovich, Steffen Marcus, and Jonathan Wise, Comparison theorems for Gromov-Witten invariants of smooth pairs and of degenerations, Ann. Inst. Fourier (Grenoble) 64 (2014), no. 4, 1611-1667. MR 3329675

[CC19] Dawei Chen and Qile Chen, Spin and hyperelliptic structures of log twisted differentials, Selecta Math. (N.S.) 25 (2019), no. 2, Paper No. 20, 42. MR 3916969

[CCUW20] Renzo Cavalieri, Melody Chan, Martin Ulirsch, and Jonathan Wise, A moduli stack of tropical curves, Forum Math. Sigma 8 (2020), Paper No. e23, 93. MR 4091085

[CJ18] Emily Clader and Felix Janda, Pixton's double ramification cycle relations, Geom. Topol. 22 (2018), no. 2, 10691108. MR 3748684

[CMW12] Renzo Cavalieri, Steffen Marcus, and Jonathan Wise, Polynomial families of tautological classes on $\mathscr{M}_{g, n}^{r t}$, J. Pure Appl. Algebra 216 (2012), no. 4, 950-981. MR 2864866 (2012j:14019)

[FP18] Gavril Farkas and Rahul Pandharipande, The moduli space of twisted canonical divisors, J. Inst. Math. Jussieu 17 (2018), no. 3, 615-672. MR 3789183

[Gil12] W. D. Gillam, Logarithmic stacks and minimality, Internat. J. Math. 23 (2012), no. 7, 1250069, 38. MR 2945649

[Gro05] Alexander Grothendieck, Cohomologie locale des faisceaux cohérents et théorèmes de Lefschetz locaux et globaux (SGA 2), Documents Mathématiques (Paris) [Mathematical Documents (Paris)], vol. 4, Société Mathématique de France, Paris, 2005, Séminaire de Géométrie Algébrique du Bois Marie, 1962, Augmenté d'un exposé de Michèle Raynaud. [With an exposé by Michèle Raynaud], With a preface and edited by Yves Laszlo, Revised reprint of the 1968 French original. MR 2171939

[Gué16] Jérémy Guéré, A generalization of the double ramification cycle via log-geometry, March 2016, arXiv:1603.09213.

[GV05] Tom Graber and Ravi Vakil, Relative virtual localization and vanishing of tautological classes on moduli spaces of curves, Duke Math. J. 130 (2005), no. 1, 1-37. MR 2176546 (2006j:14035)

[GZ12a] Samuel Grushevsky and Dmitry Zakharov, The double ramification cycle and the theta divisor.

[GZ12b] The zero section of the universal semiabelian variety, and the double ramification cycle.

[Hai11] R. Hain, Normal functions and the geometry of moduli spaces of curves, Handbook of Moduli (Gavril Farkas and Ian Morrison, editors), arXiv:1102.4031, 2011.

[Hol17] David Holmes, Extending the double ramification cycle by resolving the Abel-Jacobi map, July 2017, arXiv: 1707.02261

[Hol19] _ Néron models of jacobians over base schemes of dimension greater than 1, J. Reine Angew. Math. 747 (2019), 109-145. MR 3905131

[IKNU20] Tetsushi Ito, Kazuya Kato, Chikara Nakayama, and Sampei Usui, On log motives, Tunis. J. Math. 2 (2020), no. 4, 733-789. MR 4043075

[JPPZ17] F. Janda, R. Pandharipande, A. Pixton, and D. Zvonkine, Double ramification cycles on the moduli spaces of curves, Publications mathématiques de l'IHÉS 125 (2017), no. 1, 221-266.

[Kat89] Kazuya Kato, Logarithmic structures of Fontaine-Illusie, Algebraic analysis, geometry, and number theory (Baltimore, MD, 1988), Johns Hopkins Univ. Press, Baltimore, MD, 1989, pp. 191-224. MR 1463703

[Kat94] Toric singularities, Amer. J. Math. 116 (1994), no. 5, 1073-1099. MR 1296725

[Kat00] Fumiharu Kato, Log smooth deformation and moduli of log smooth curves, Internat. J. Math. 11 (2000), no. 2, 215-232. MR 1754621

[Li01] Jun Li, Stable morphisms to singular schemes and relative stable morphisms, J. Differential Geom. 57 (2001), no. 3, 509-578. MR 1882667

[Li02] _ A degeneration formula of GW-invariants, J. Differential Geom. 60 (2002), no. 2, 199-293. MR 1938113

[Man12] Cristina Manolache, Virtual pull-backs, J. Algebraic Geom. 21 (2012), no. 2, 201-245. MR 2877433

[Mil80] James S. Milne, Étale cohomology, Princeton Mathematical Series, vol. 33, Princeton University Press, Princeton, N.J., 1980. MR 559531

[Mum08] David Mumford, Abelian varieties, Tata Institute of Fundamental Research Studies in Mathematics, vol. 5, Published for the Tata Institute of Fundamental Research, Bombay; by Hindustan Book Agency, New Delhi, 2008, With appendices by C. P. Ramanujam and Yuri Manin, Corrected reprint of the second (1974) edition. MR 2514037

[MW13] Steffen Marcus and Jonathan Wise, Stable maps to rational curves and the relative jacobian, October 2013, arXiv: 1310.5981.

[MW18] Samouil Molcho and Jonathan Wise, The logarithmic Picard group and its tropicalization, July 2018, arXiv: 1807.11364 .

[Ogu18] Arthur Ogus, Lectures on logarithmic algebraic geometry, vol. 178, Cambridge University Press, 2018.

[Ols03] Martin C. Olsson, Logarithmic geometry and algebraic stacks, Ann. Sci. École Norm. Sup. (4) 36 (2003), no. 5, 747-791. MR 2032986

[Ols05] _ The logarithmic cotangent complex, Math. Ann. 333 (2005), no. 4, 859-931. MR 2195148 
[RSPW19] Dhruv Ranganathan, Keli Santos-Parker, and Jonathan Wise, Moduli of stable maps in genus one and logarithmic geometry, I, Geom. Topol. 23 (2019), no. 7, 3315-3366. MR 4046967

[RW20] Dhruv Ranganathan and Jonathan Wise, Rational curves in the logarithmic multiplicative group, Proc. Amer. Math. Soc. 148 (2020), no. 1, 103-110. MR 4042834

[Sch18] Johannes Schmitt, Dimension theory of the moduli space of twisted $k$-differentials, Doc. Math. 23 (2018), 871-894. MR 3861042

[Tsu97] Takeshi Tsuji, Saturated morphisms of logarithmic schemes, June 1997.

[Wis16] Jonathan Wise, Moduli of morphisms of logarithmic schemes, Algebra Number Theory 10 (2016), no. 4, 695-735. MR 3519093

(Marcus) Mathematics and Statistics, The College of New Jersey, Ewing, NJ 08628, USA

Email address: marcuss@tcnj.edu

(Wise) University of Colorado, Boulder, Boulder, Colorado 80309-0395, USA

Email address: jonathan.wise@math.colorado.edu 